\title{
Intramolecular Interactions between Chalcogen Atoms: Organoseleniums Derived from 1-Bromo-4-tert-butyl-2,6- di(formyl)benzene
}

Sanjio S. Zade, ${ }^{a}$ Snigdha Panda,${ }^{a}$ Harkesh B. Singh,${ }^{* a}$ Raghavan B. Sunoj, ${ }^{a}$ Ray J. Butcher ${ }^{b}$

${ }^{\mathrm{a}}$ Department of Chemistry, Indian Institute of Technology Bombay, Powai, Mumbai 400076 India

${ }^{b}$ Department of Chemistry, Howard University, Washington D. C. 20059, USA

Address correspondence to

Prof. Harkesh B. Singh

Tel: 091-022-25767190

Fax: 091-22-2572 3480

E-mail: chhbsia@chem.iitb.ac.in 


\section{CONTENTS}

Page No.

1. General Procedures $\quad$ S3

2. ${ }^{1} \mathrm{H},{ }^{13} \mathrm{C}$ and ${ }^{77}$ Se spectra of $23,25-30$ and $34 \quad$ S4-S27

3. The B3LYP/6-31G* optimized geometries and total energies for various structures

S28-S30

4. Details on computed NPA charges on the oxygen and selenium atoms at the NPA/B3LYP/6-31G*//B3LYP/6-31G* level

5. Details of lone-pair delocalizations from oxygen and selenium computed at the NBO/B3LYP/6-31G*//B3LYP/6-31G* level.

6. Tables for crystallographic data of $25,26,27$ and 32

7. PLUTON view of Compound 26 showing intermolecular C-H...Se interaction (Figure S25)

8. PLUTON view of Compound 34 (Figure S26)

9. Packing diagram of 34 (Figure S27)

10. ORTEP diagram of Compound 27 (Figure S28) 
General procedures: All reactions were carried out under nitrogen or argon using standard vacuumline techniques. Solvents were purified by standard procedures and were freshly distilled prior to use. Melting points were recorded in capillary tubes and uncorrected. The ${ }^{1} \mathrm{H}(299.94),{ }^{13} \mathrm{C}(75.42)$ and ${ }^{77} \mathrm{Se}(57.22)$ spectra were recorded in $\mathrm{CDCl}_{3}$ at room temperature. Chemical shift cited were referenced to TMS $\left({ }^{1} \mathrm{H},{ }^{13} \mathrm{C}\right)$ as internal and $\mathrm{Me}_{2} \mathrm{Se}\left({ }^{77} \mathrm{Se}\right)$ as external. The IR spectra were recorded as $\mathrm{KBr}$ pallets. 
Figure S.1. ${ }^{1} \mathrm{H}$ NMR spectrum of compound 23

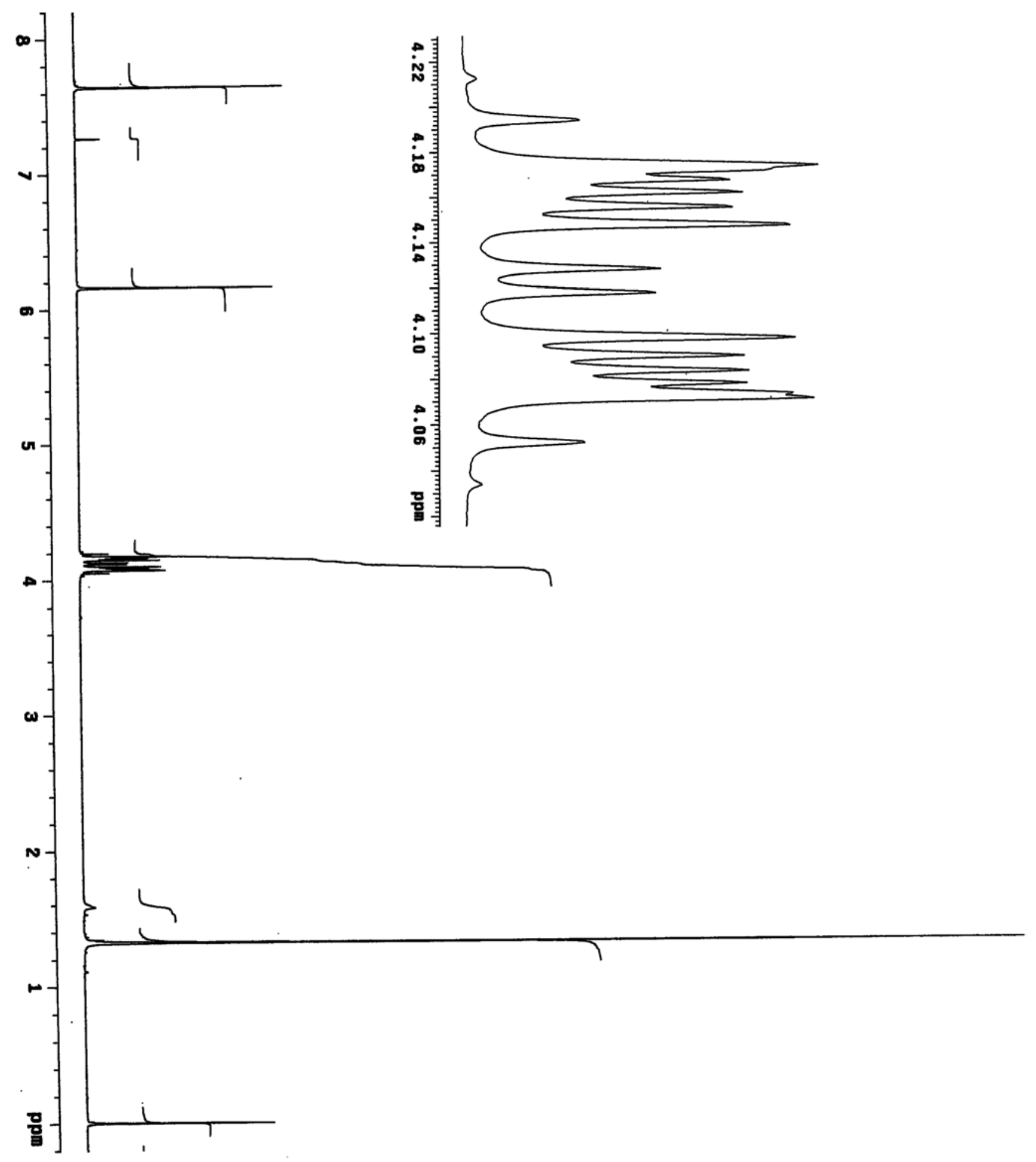


Figure S.2. ${ }^{13} \mathrm{C}$ NMR spectrum of compound 23

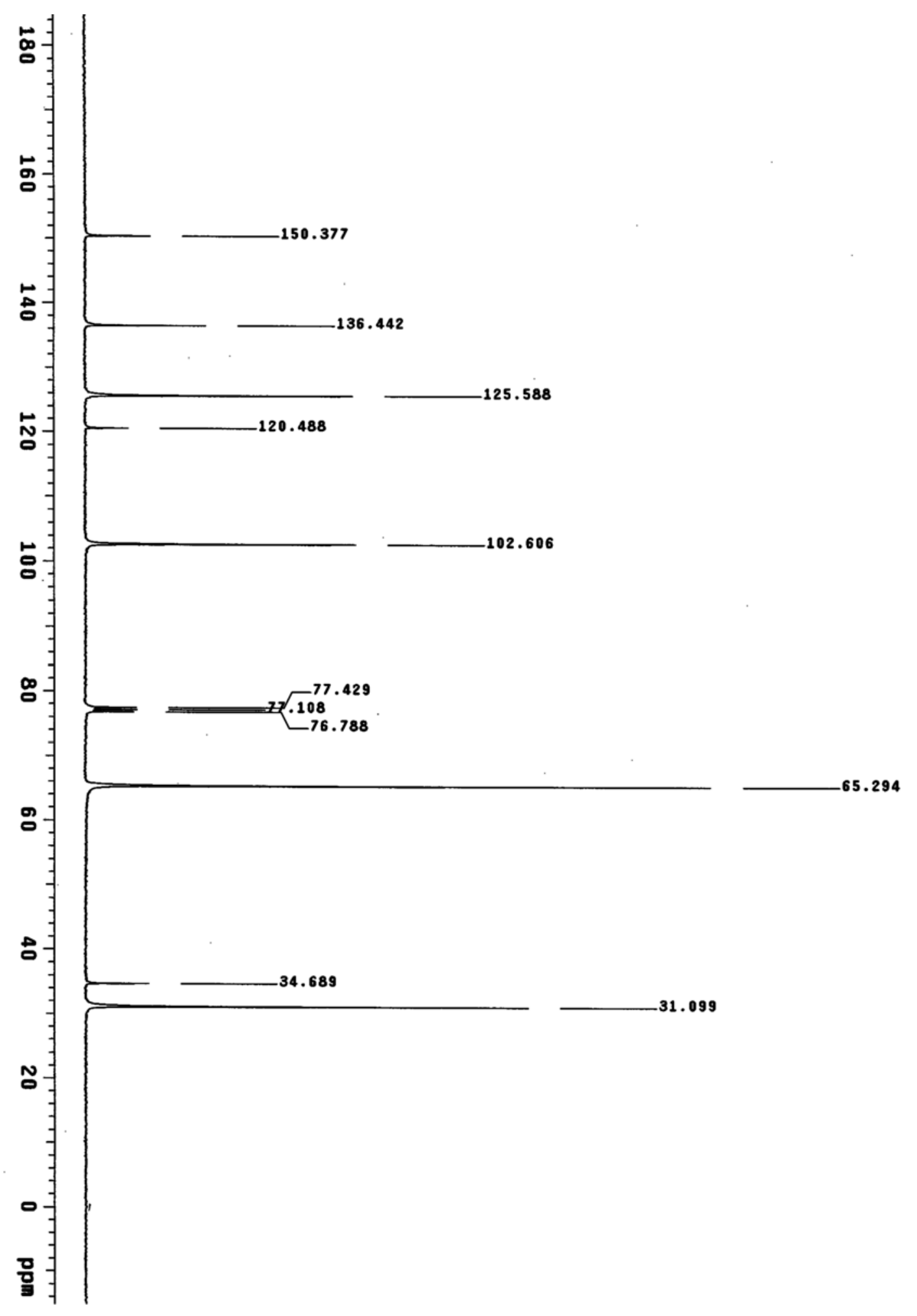


Figure S.3. ${ }^{1}$ H NMR spectrum of compound 25

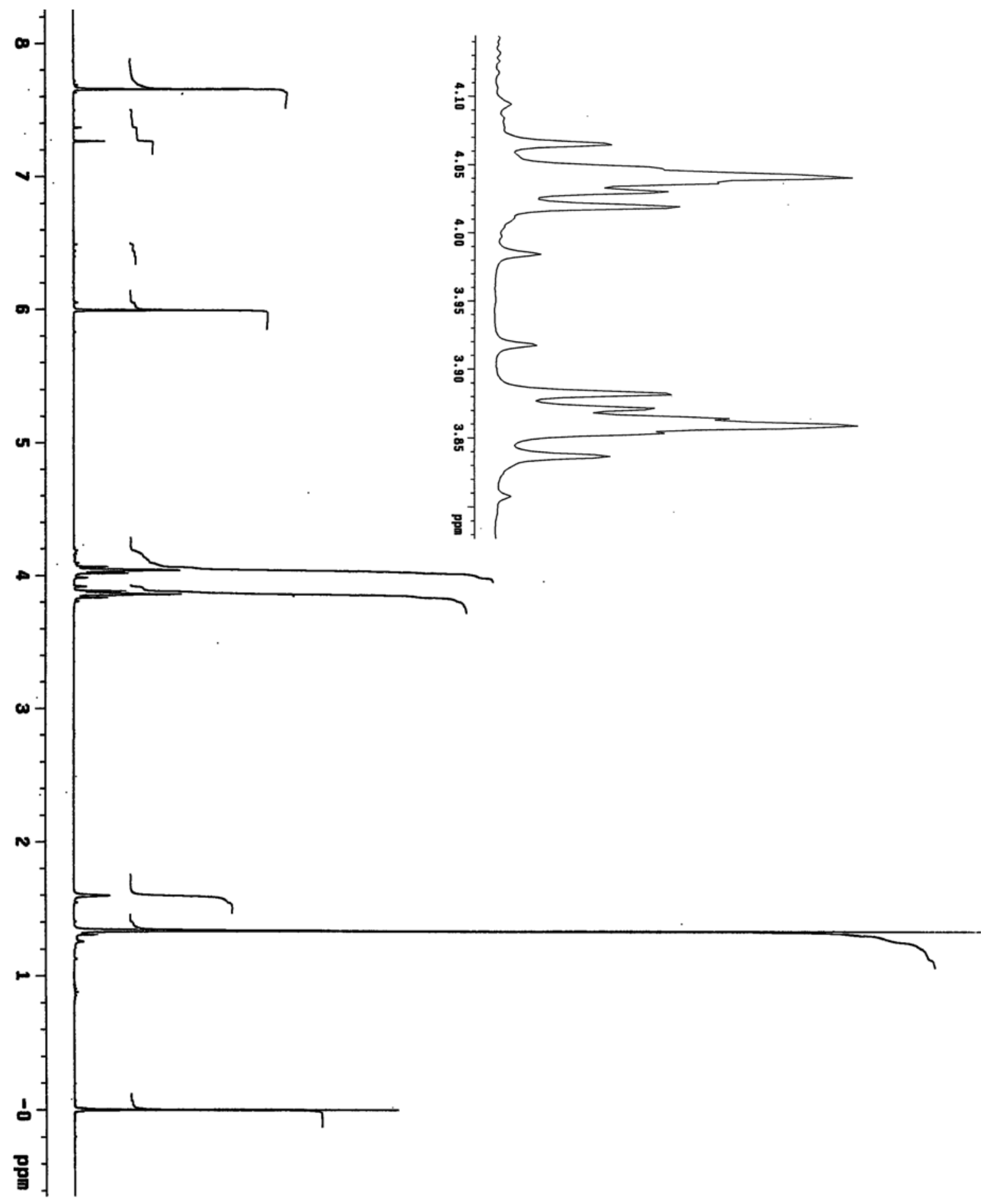

S6 
Figure S.4. ${ }^{13} \mathrm{C}$ NMR spectrum of compound 25

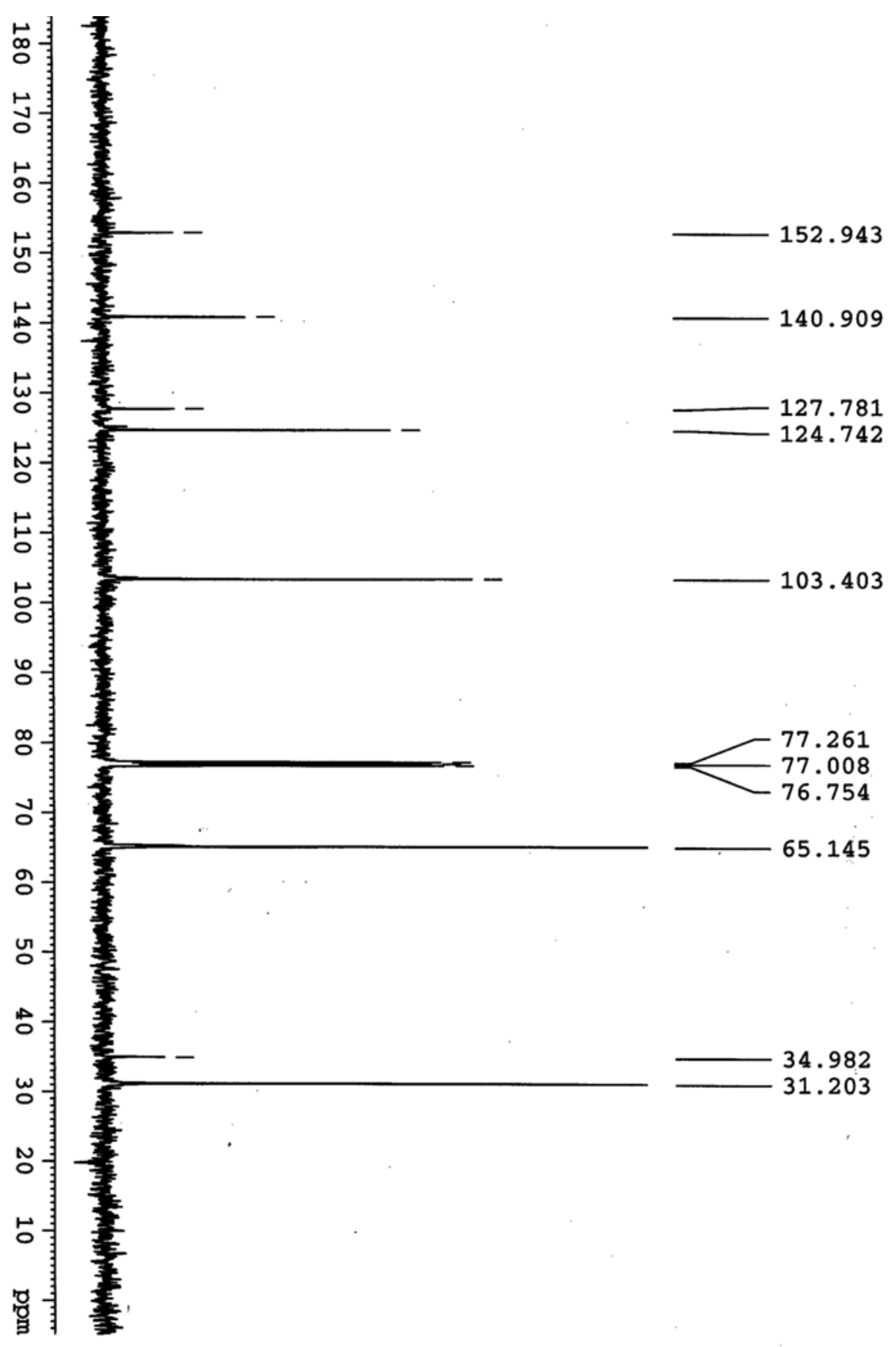


Figure S.5. ${ }^{77}$ Se NMR spectrum of compound 25

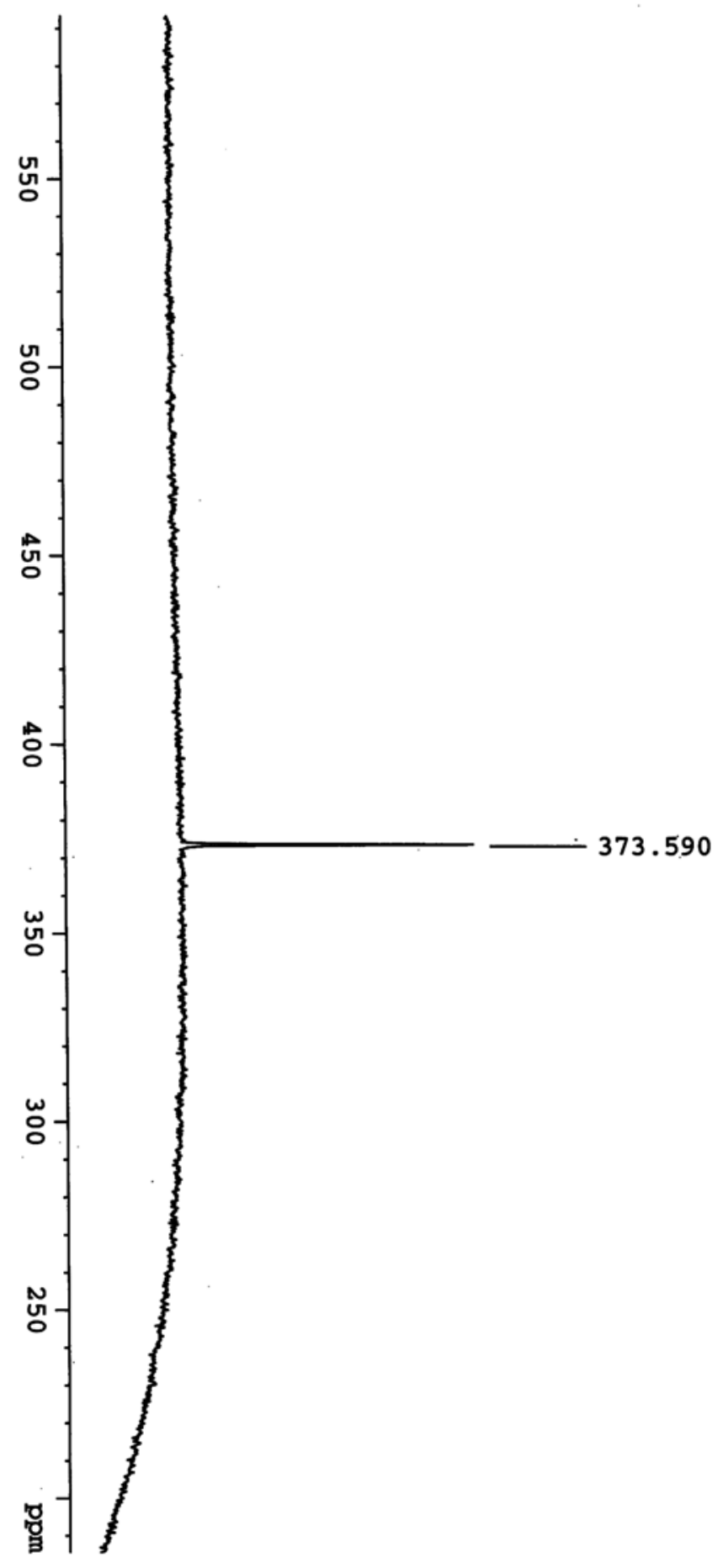


Figure S.6. ${ }^{1} \mathrm{H}$ NMR spectrum of compound 26

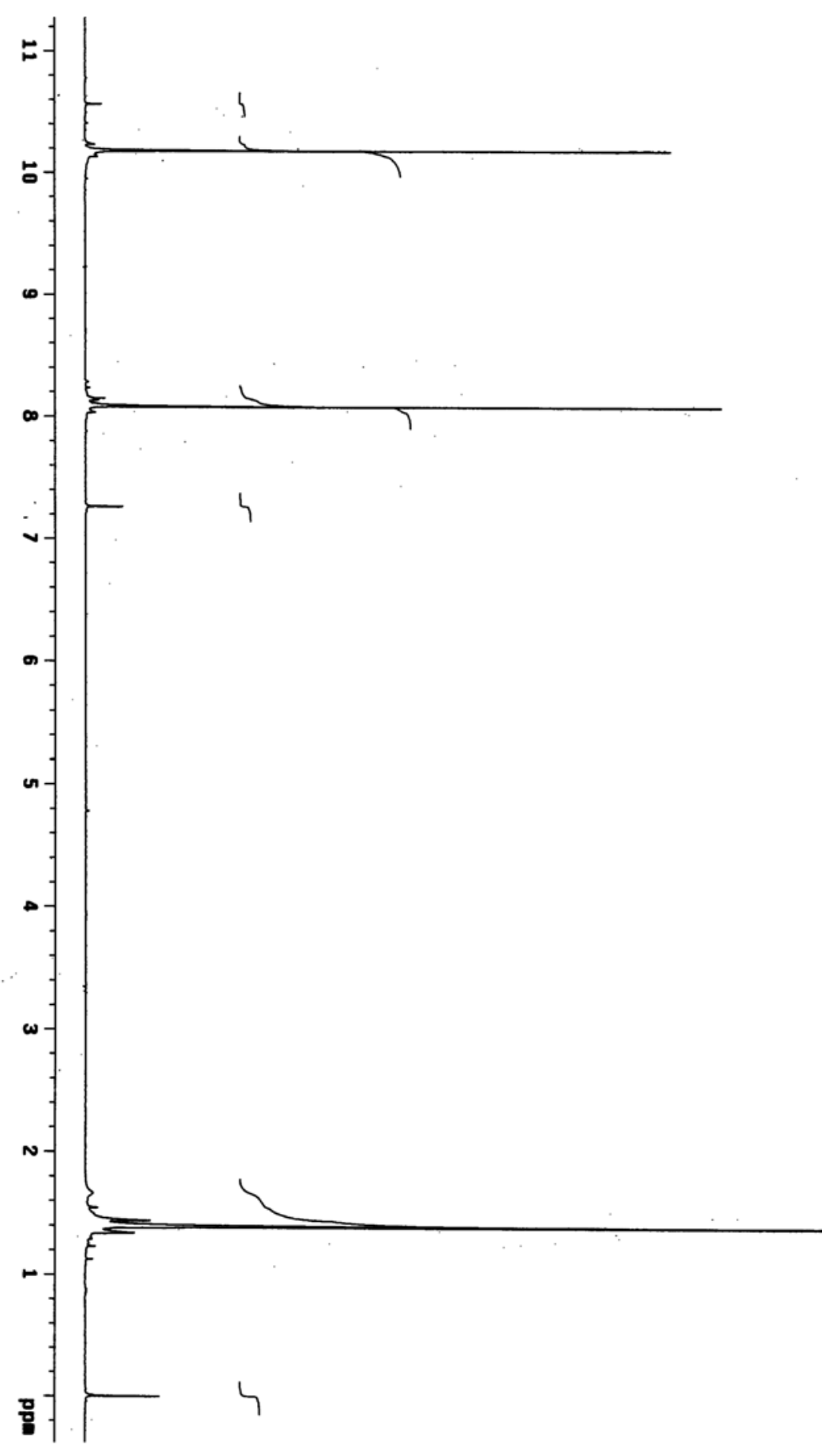


Figure S.7. ${ }^{13} \mathrm{C}$ NMR spectrum of compound 26

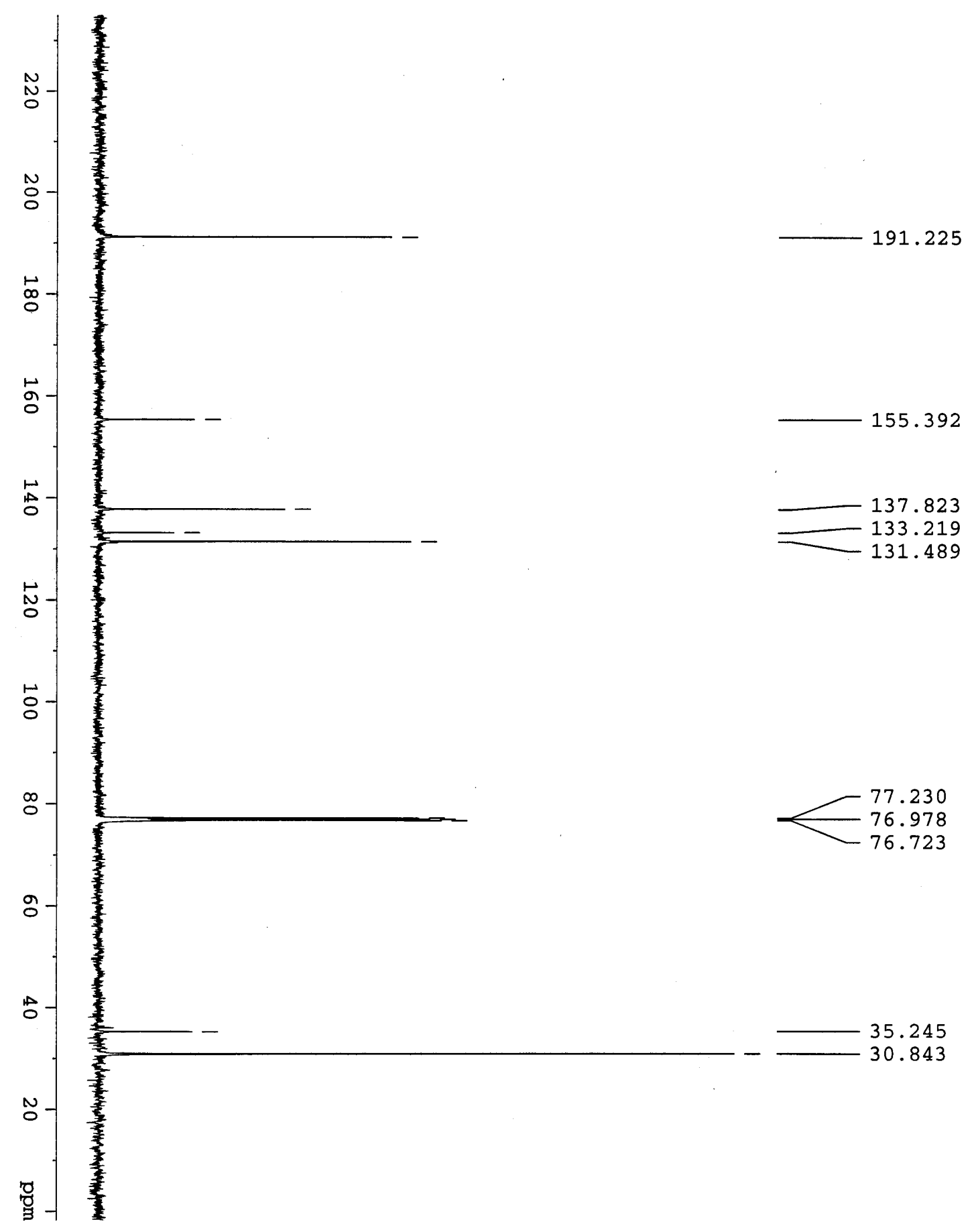


Figure S.8. ${ }^{77}$ Se NMR spectrum of compound 26

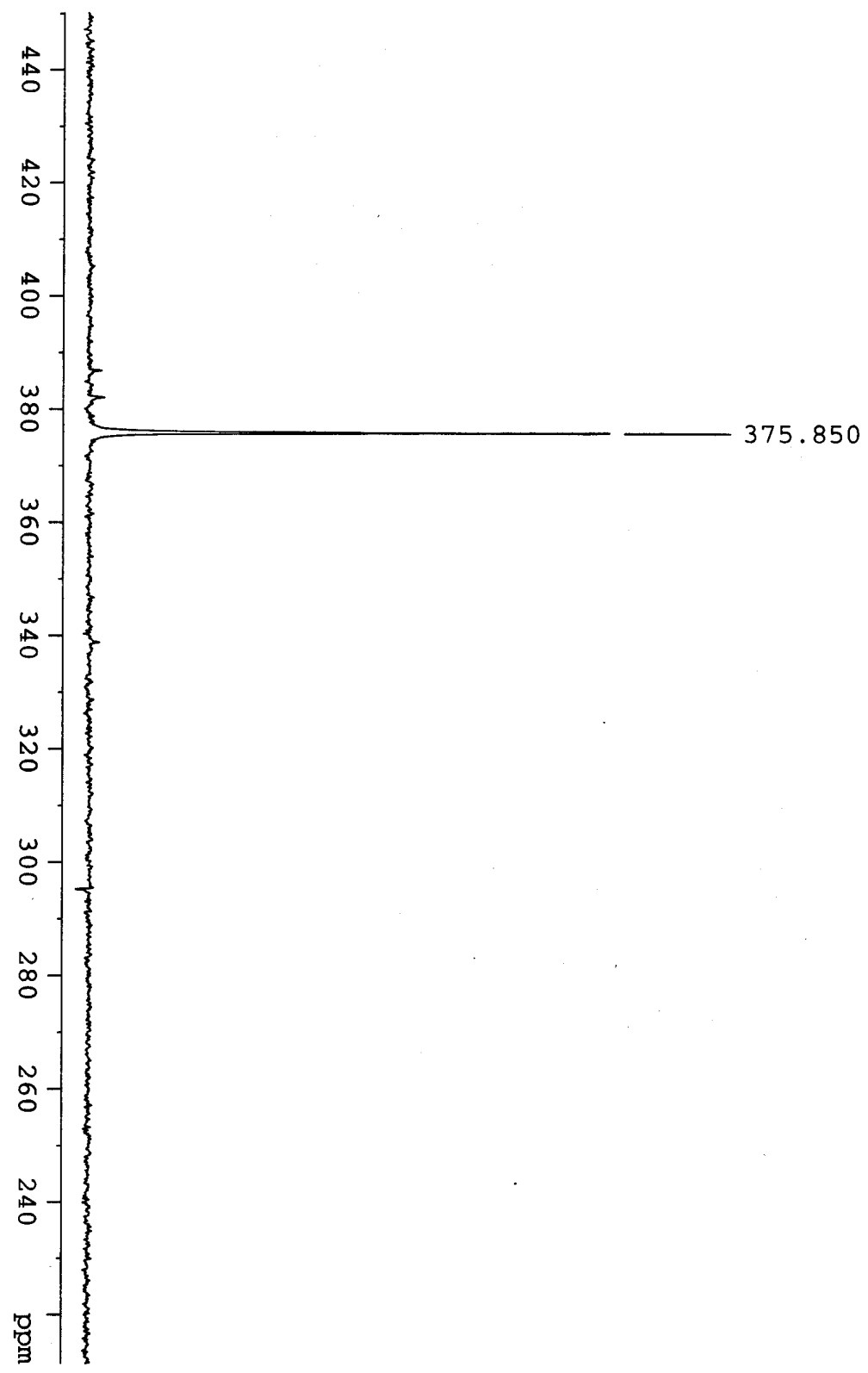


Figure S.9. ${ }^{1} \mathrm{H}$ NMR spectrum of compound 27

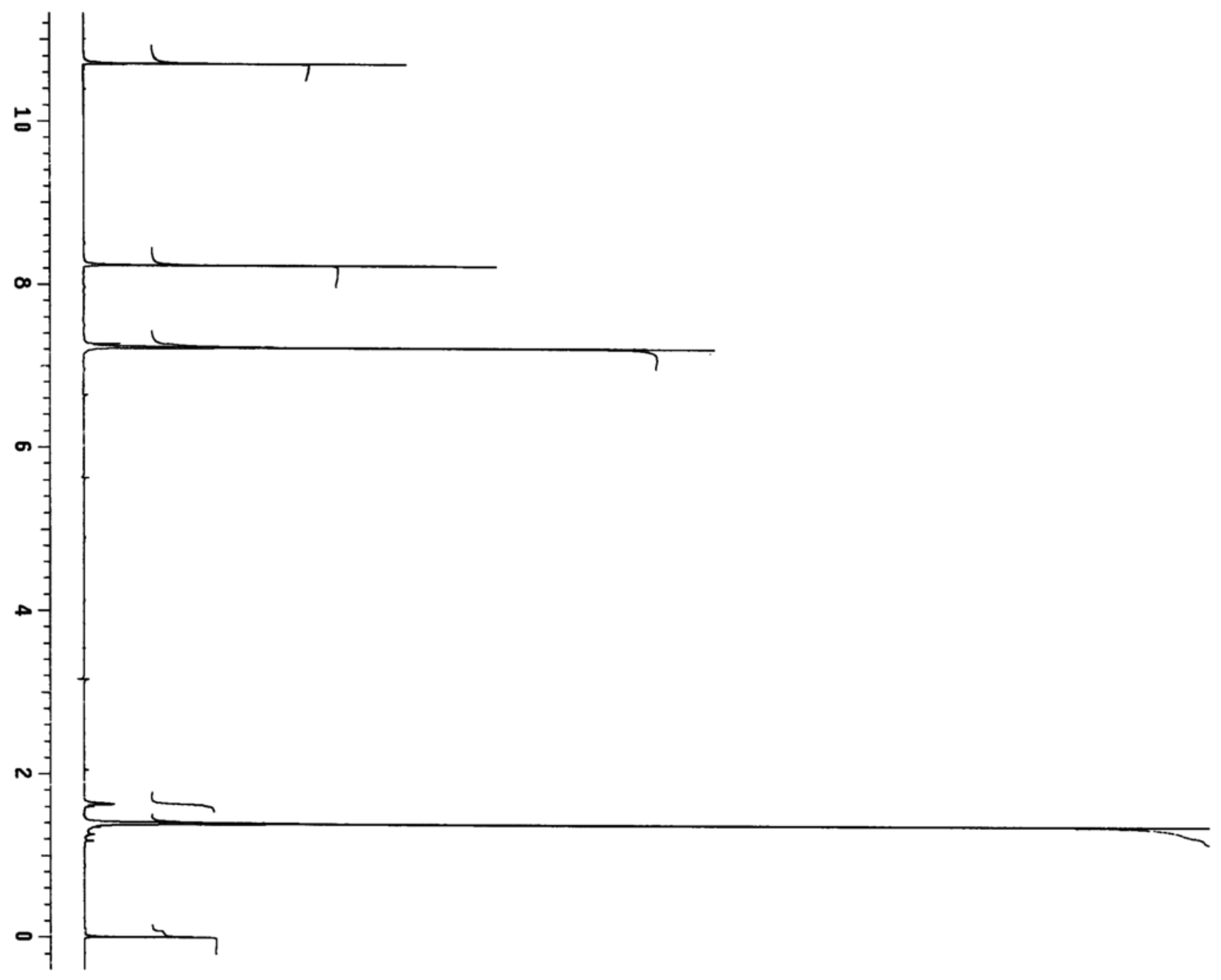


Figure S.10. ${ }^{13} \mathrm{C}$ NMR spectrum of compound 27

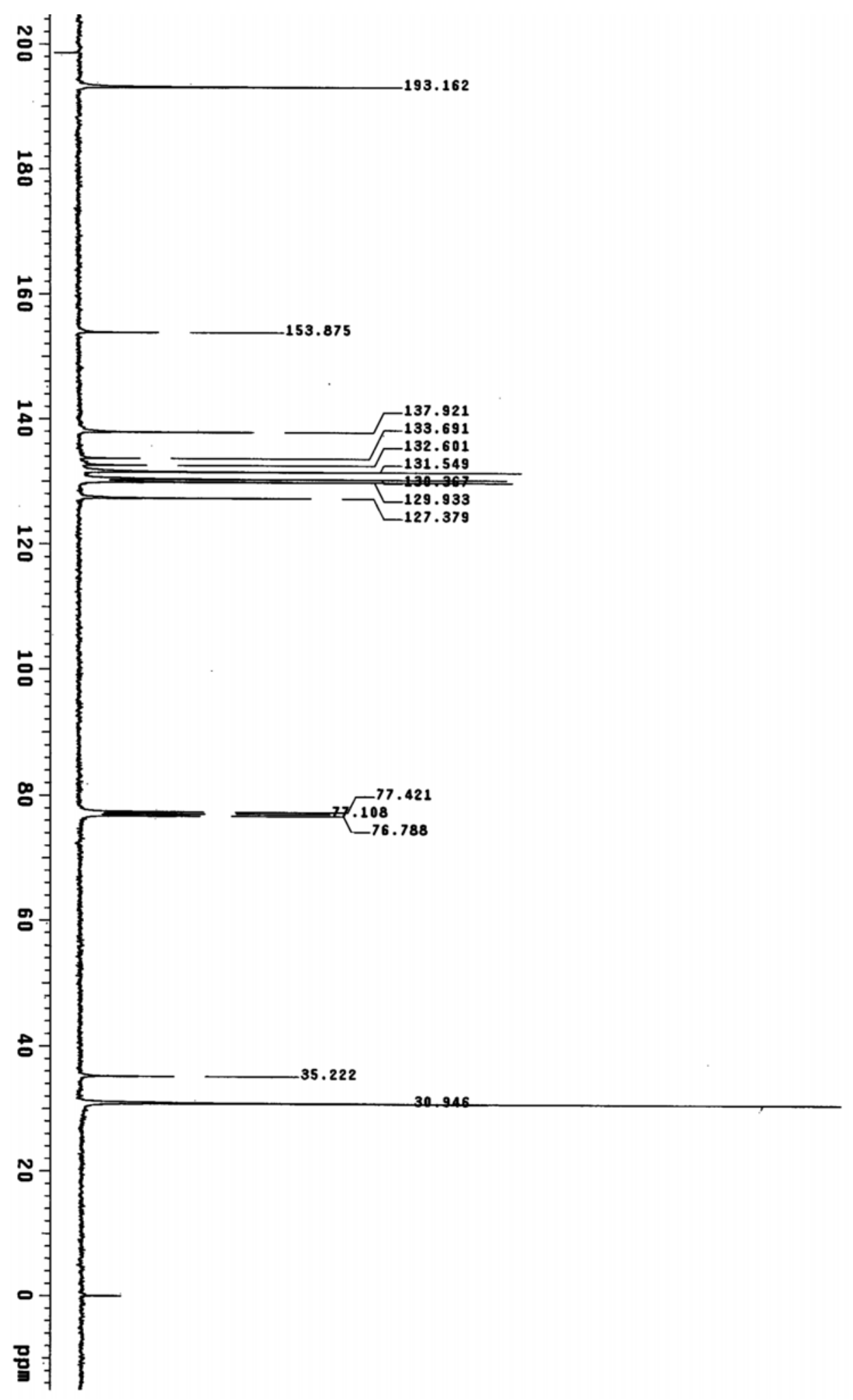


Figure S.11. ${ }^{77}$ Se NMR spectrum of compound 27

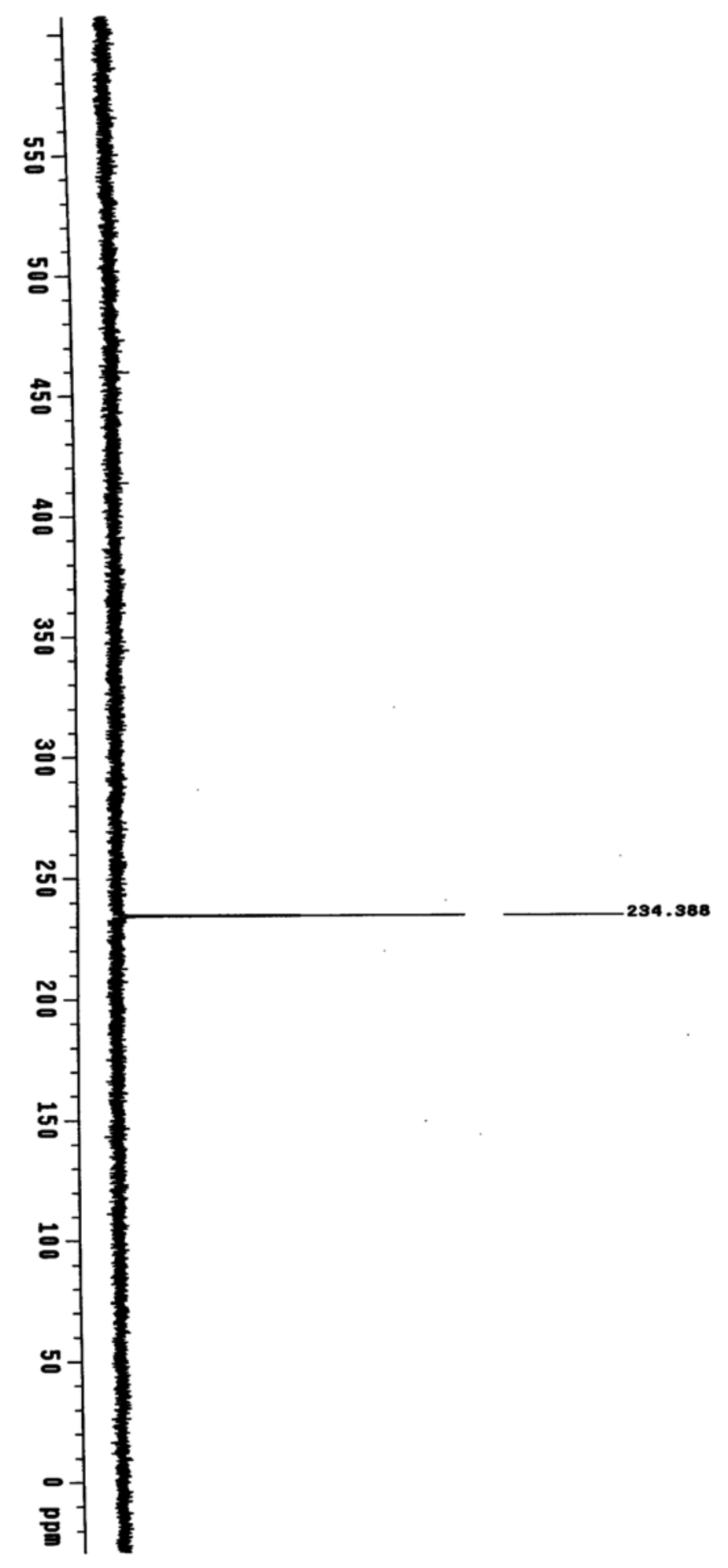


Figure S.12. ${ }^{1} \mathrm{H}$ NMR spectrum of compound 28

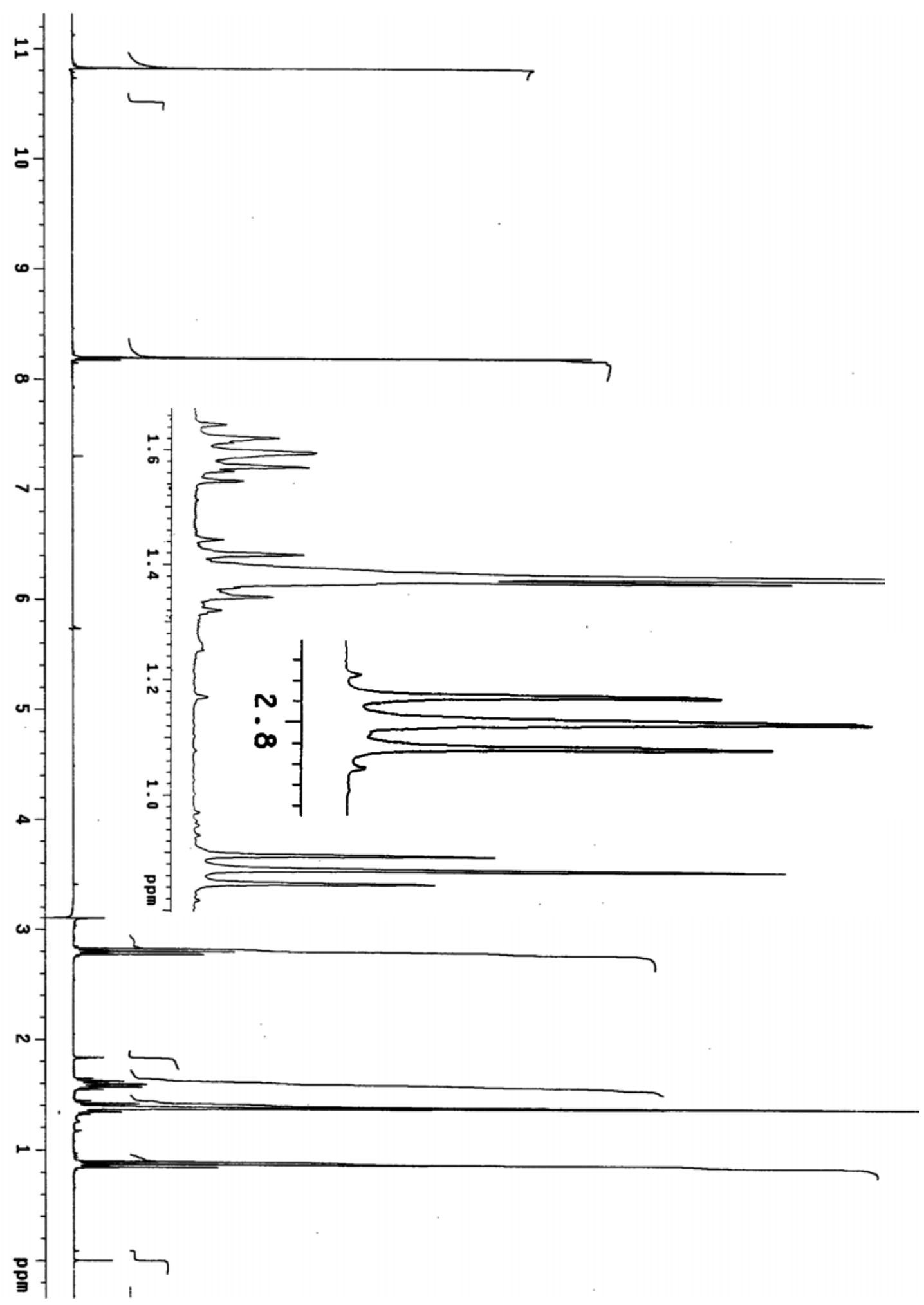


Figure S.13. ${ }^{13} \mathrm{C}$ NMR spectrum of compound 28

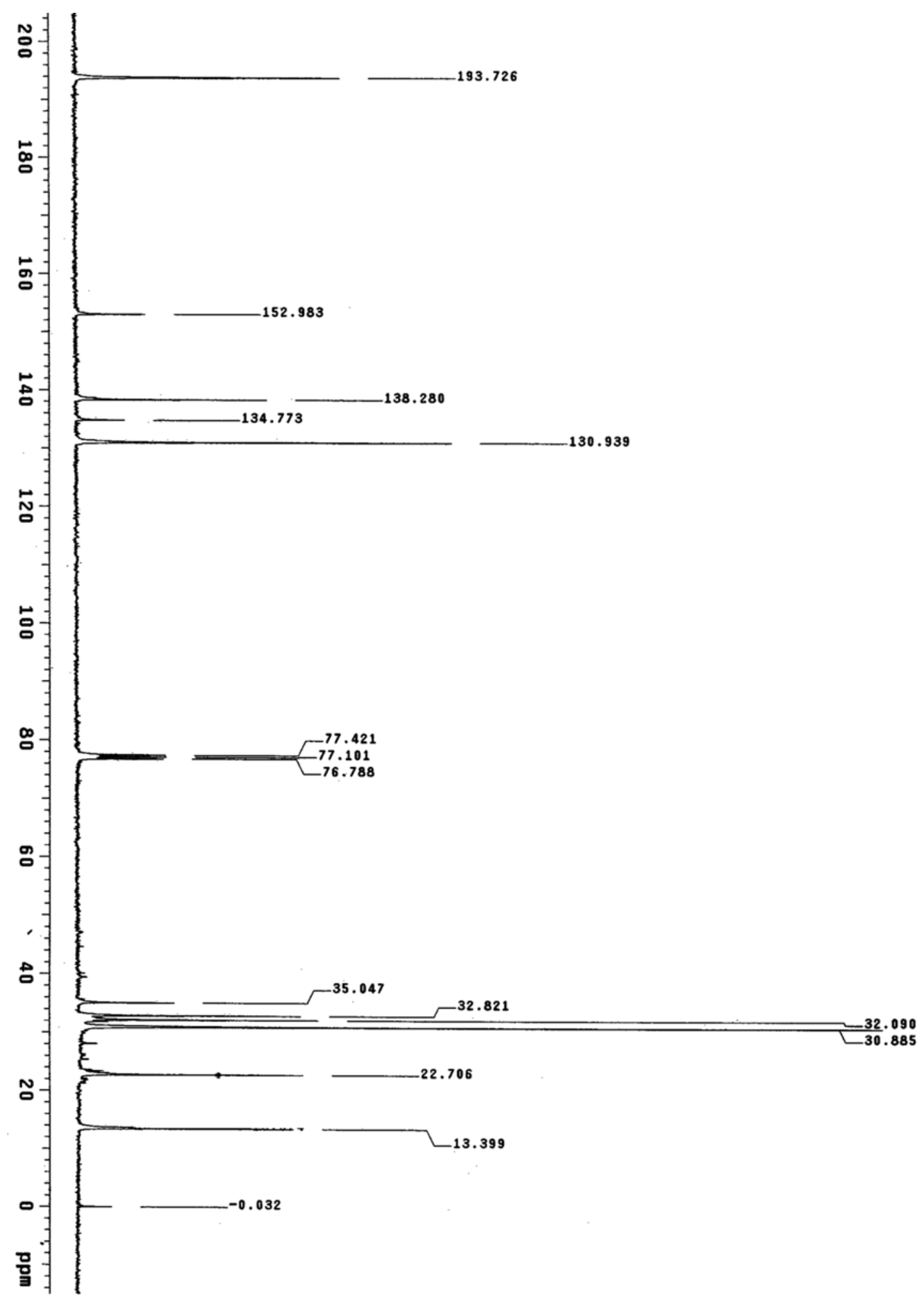


Figure S.14. ${ }^{77}$ Se NMR spectrum of compound 28

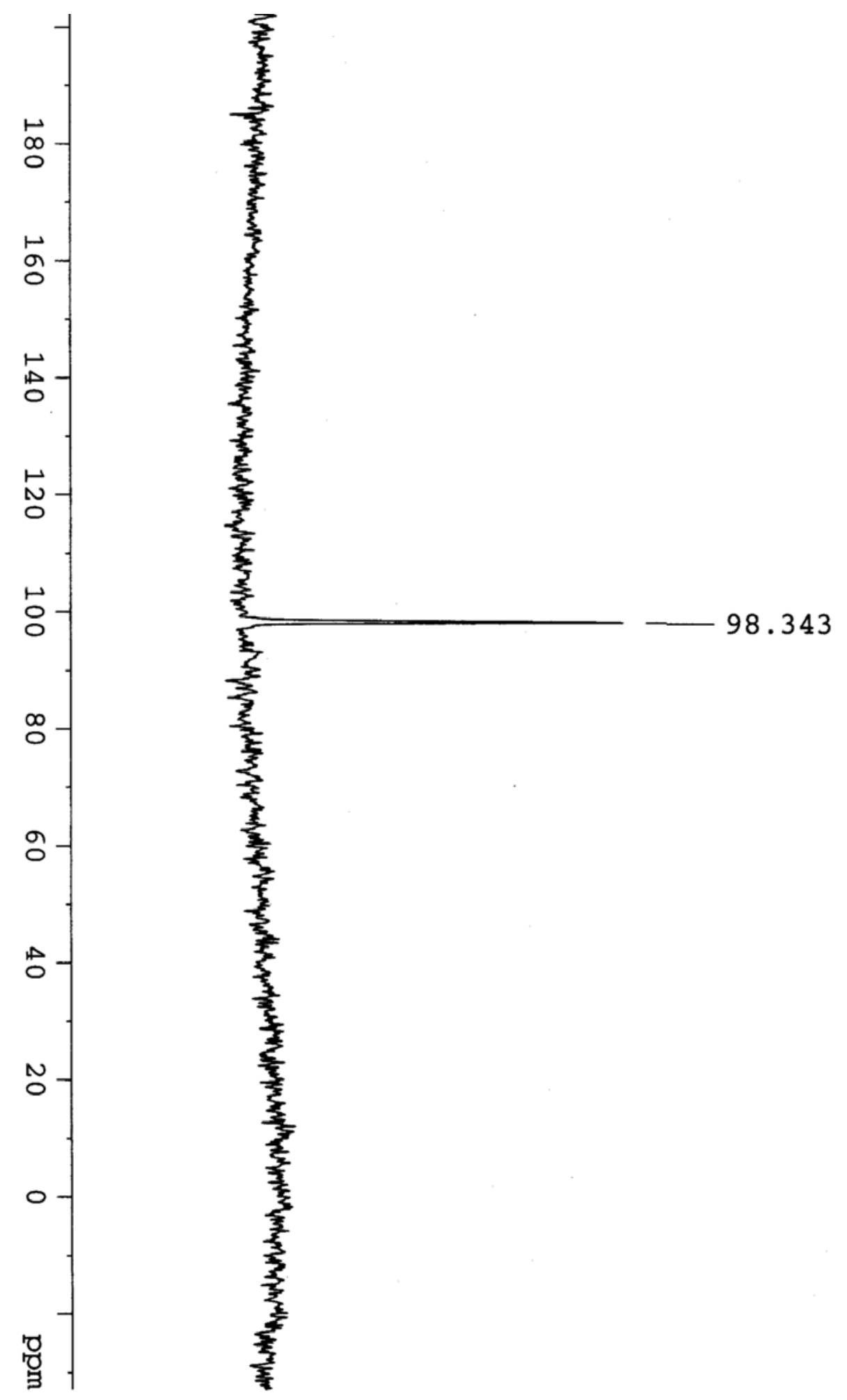


Figure S.15. ${ }^{1}$ H NMR spectrum of compound 29

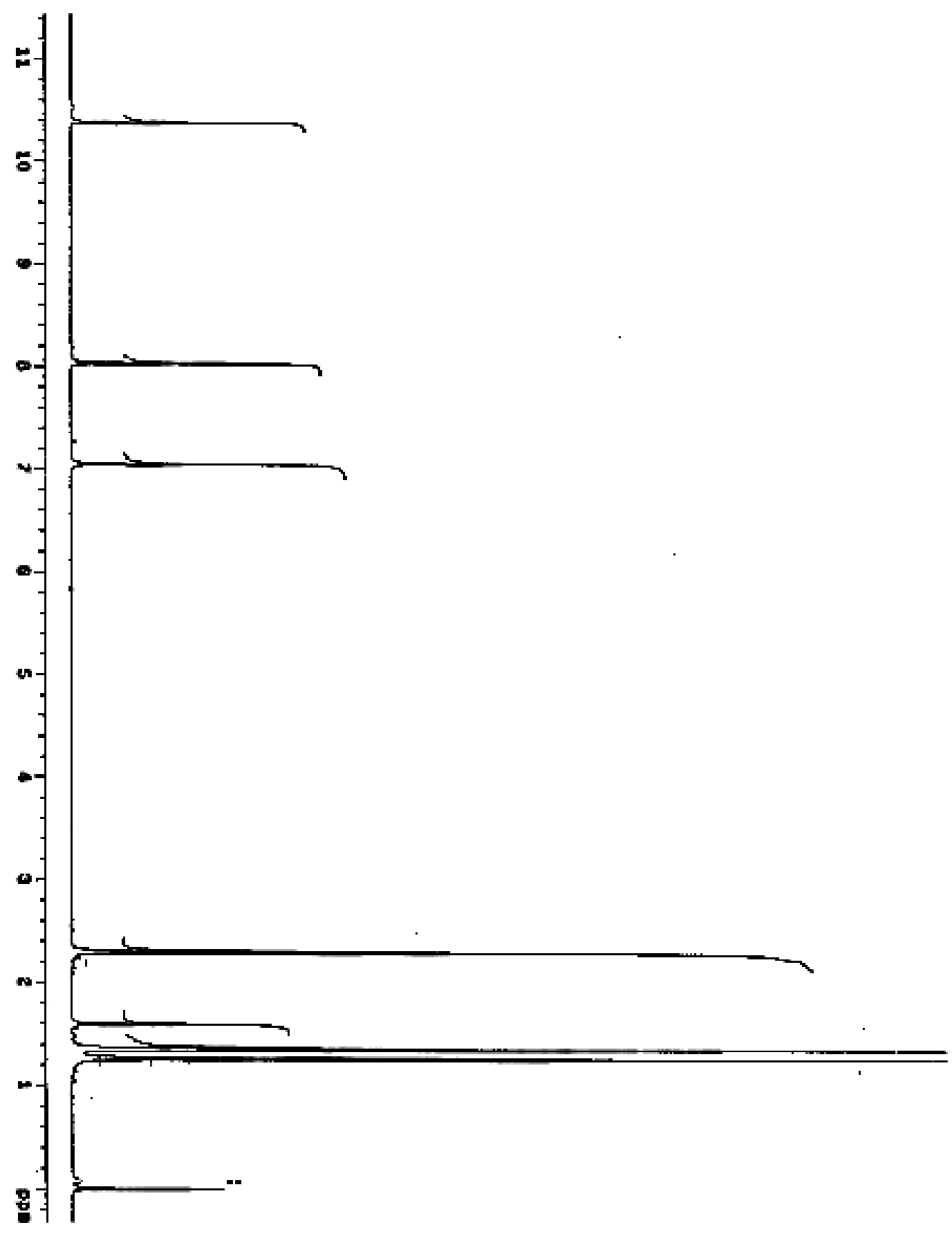


Figure S.16. ${ }^{13} \mathrm{C}$ NMR spectrum of compound 29

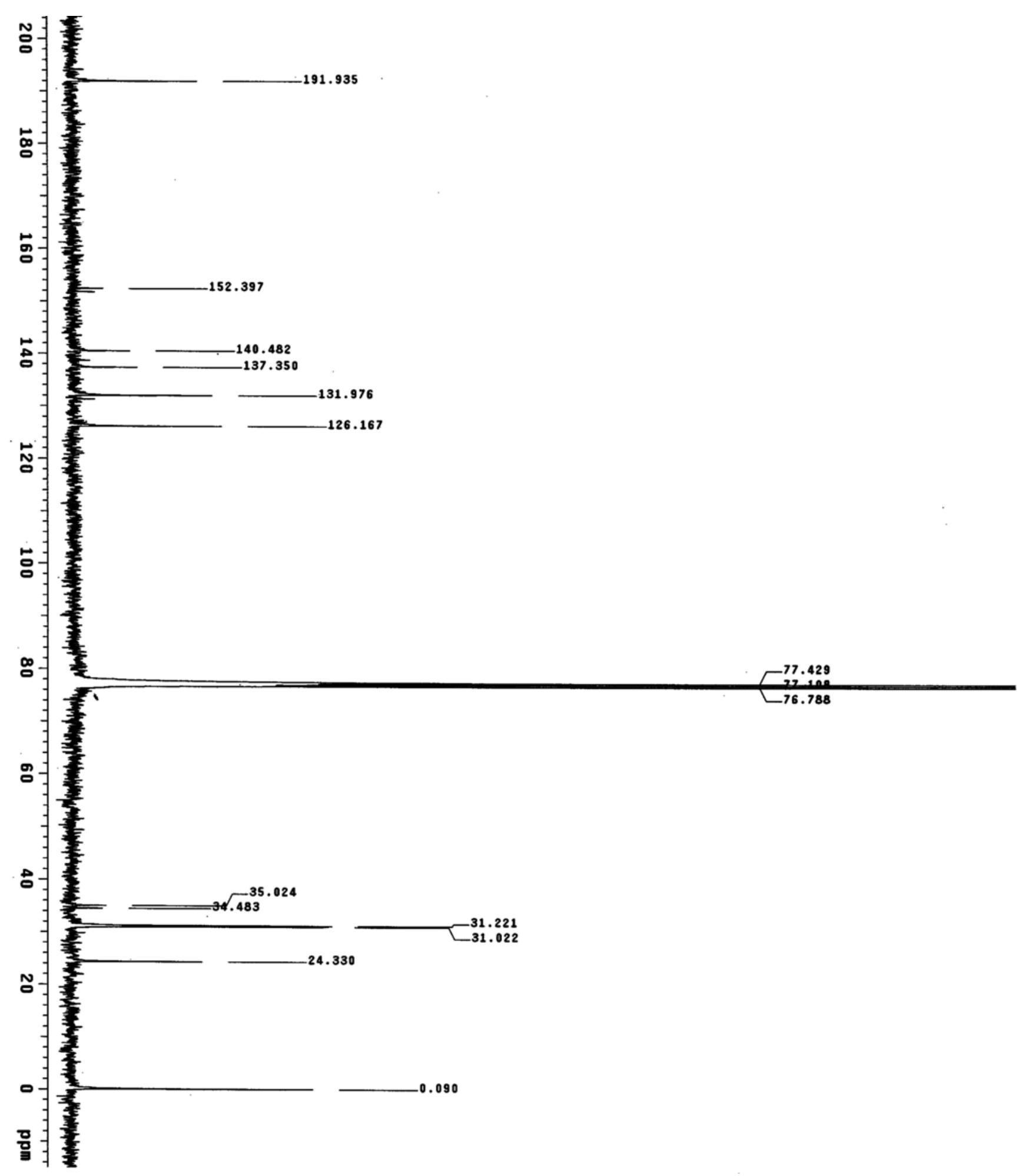


Figure S.17. ${ }^{77}$ Se NMR spectrum of compound 29

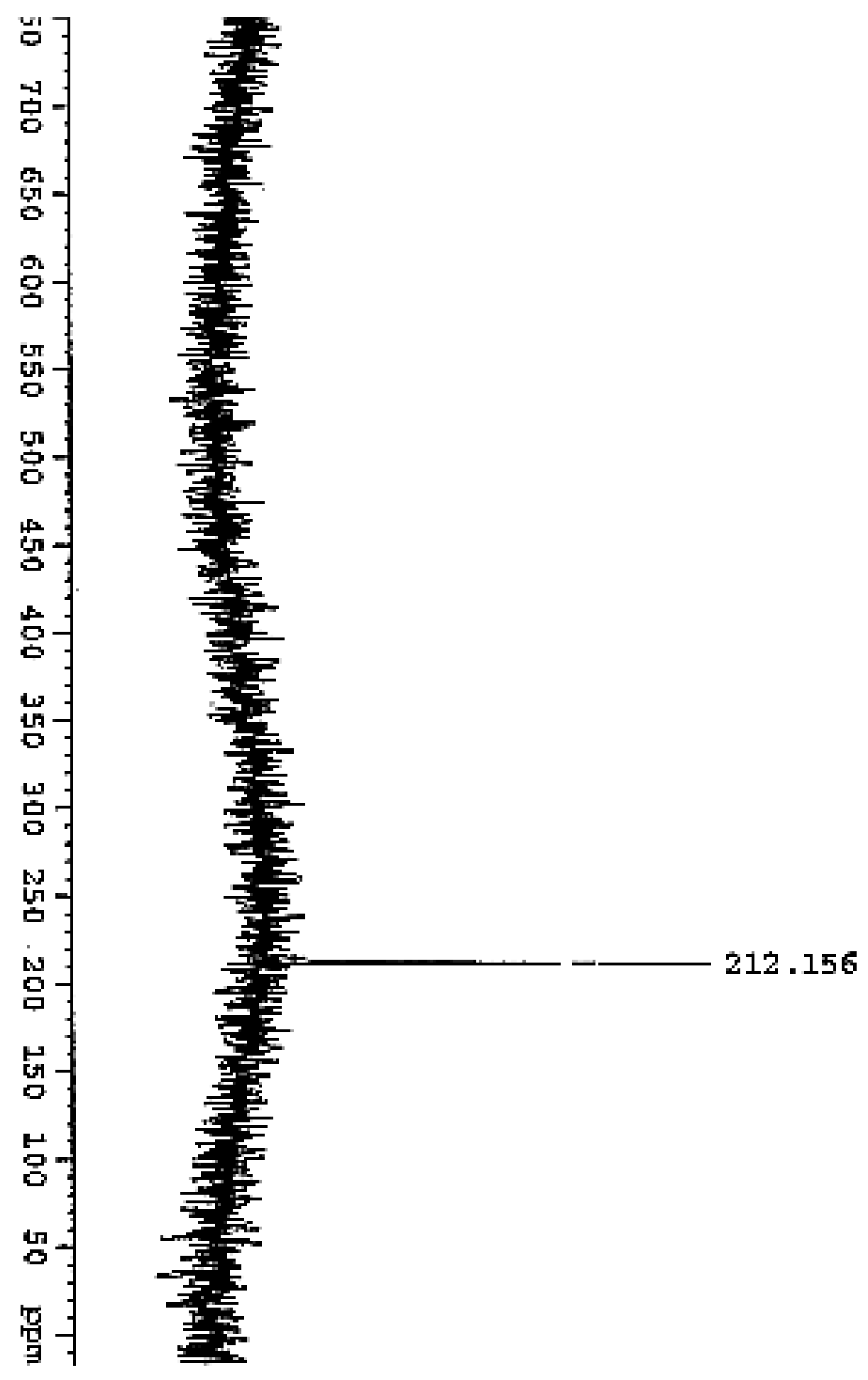


Figure S.18. ${ }^{1} \mathrm{H}$ NMR spectrum of compound 30

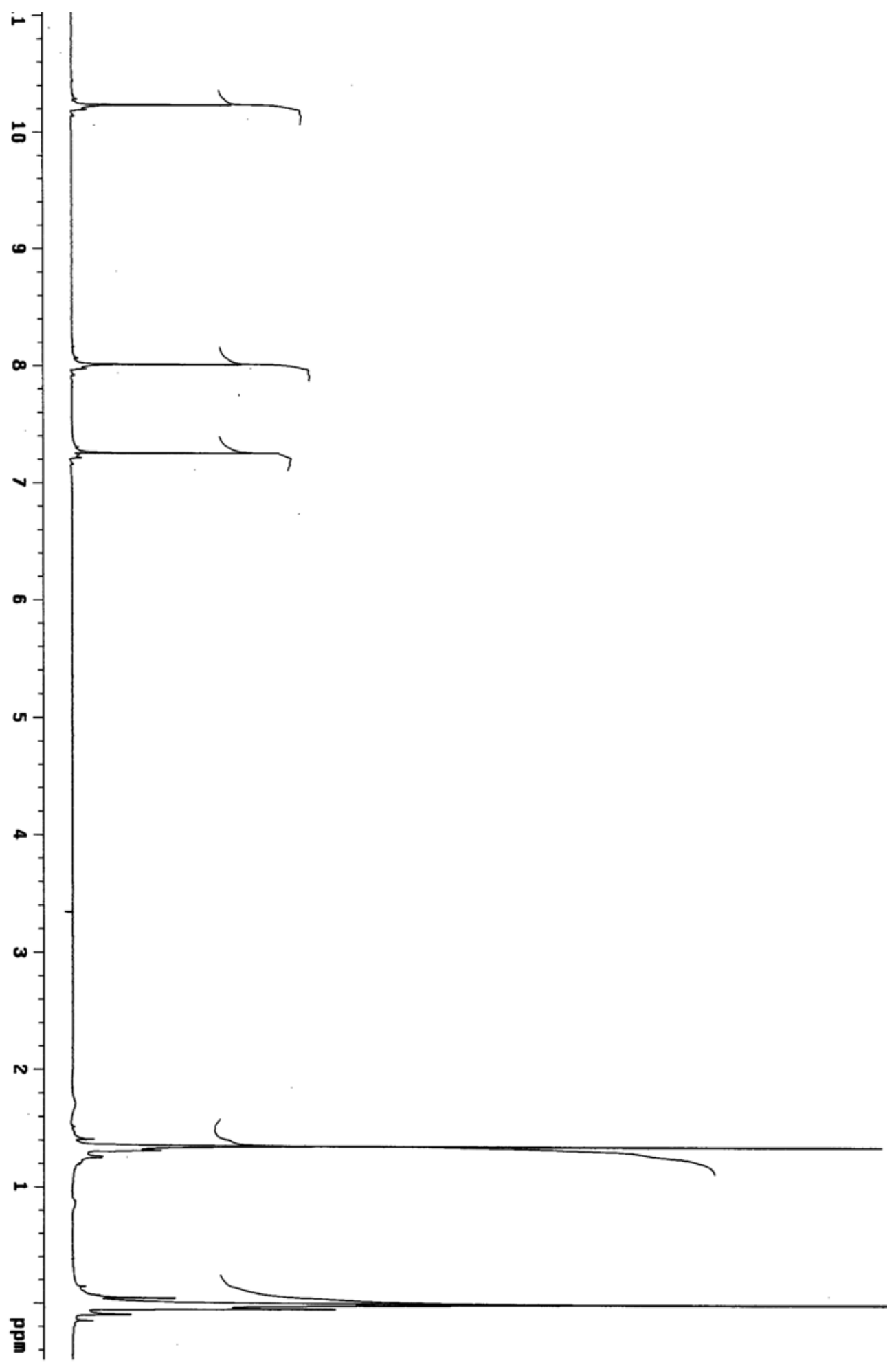


Figure S.19. ${ }^{13} \mathrm{C}$ NMR spectrum of compound 30

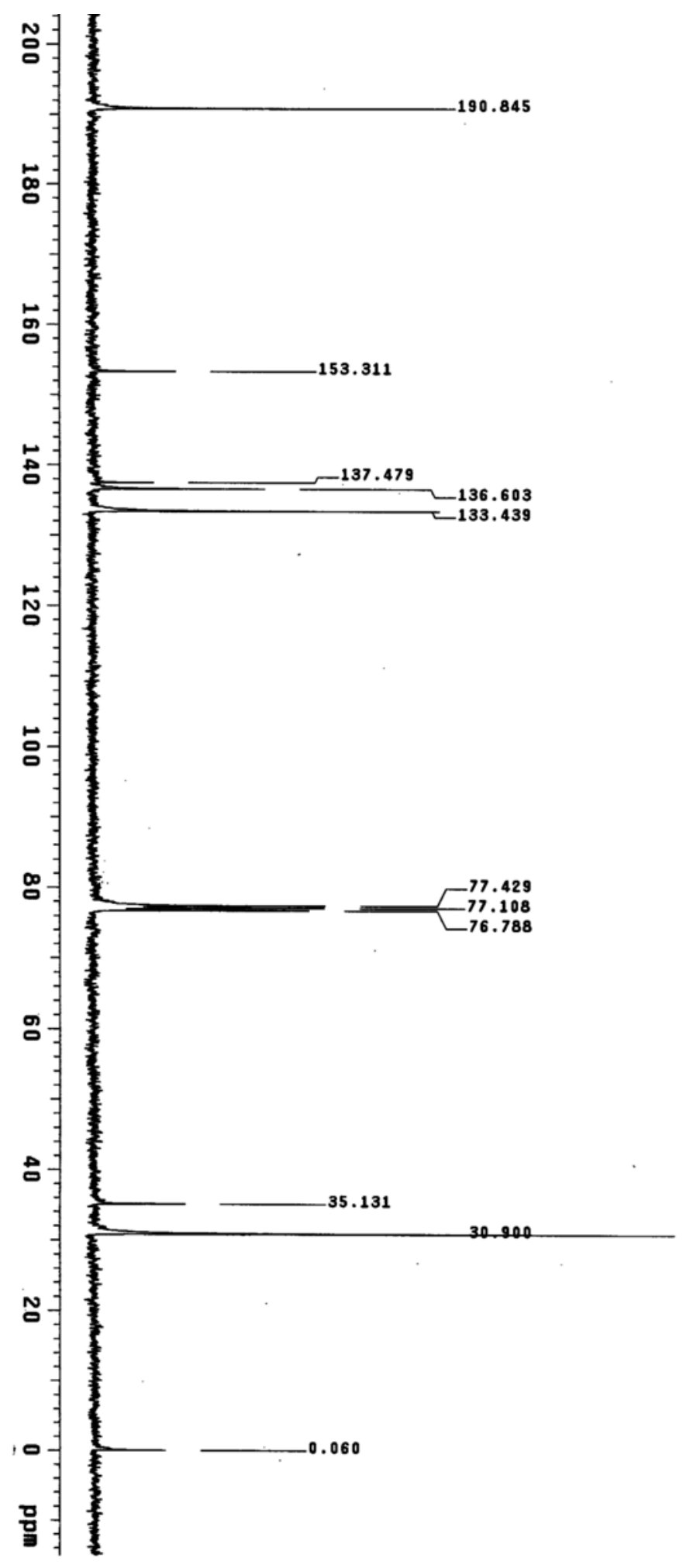


Figure S.20. ${ }^{77}$ Se NMR spectrum of compound 30

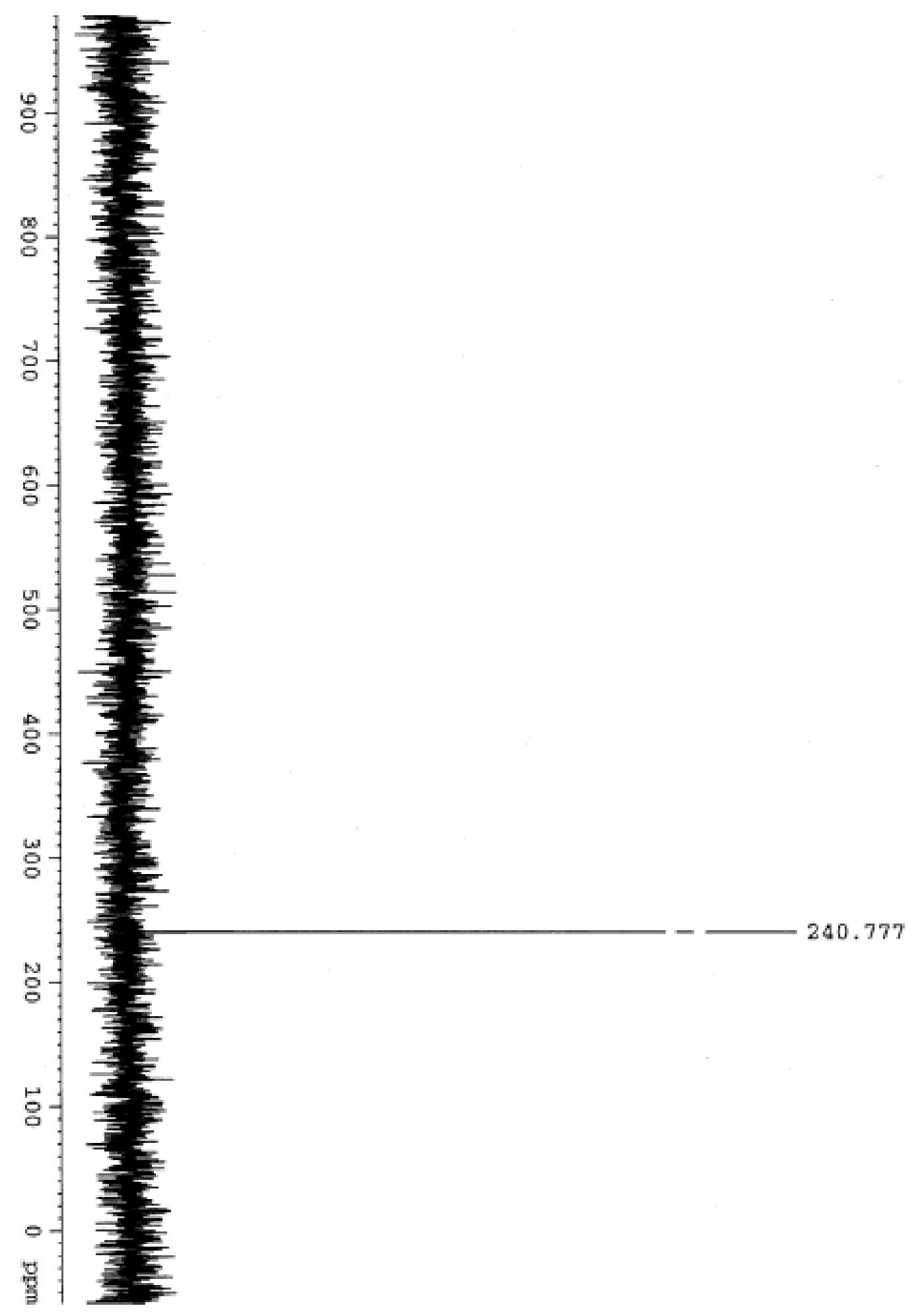


Figure S.21. ${ }^{1}$ H NMR spectrum of compound 34

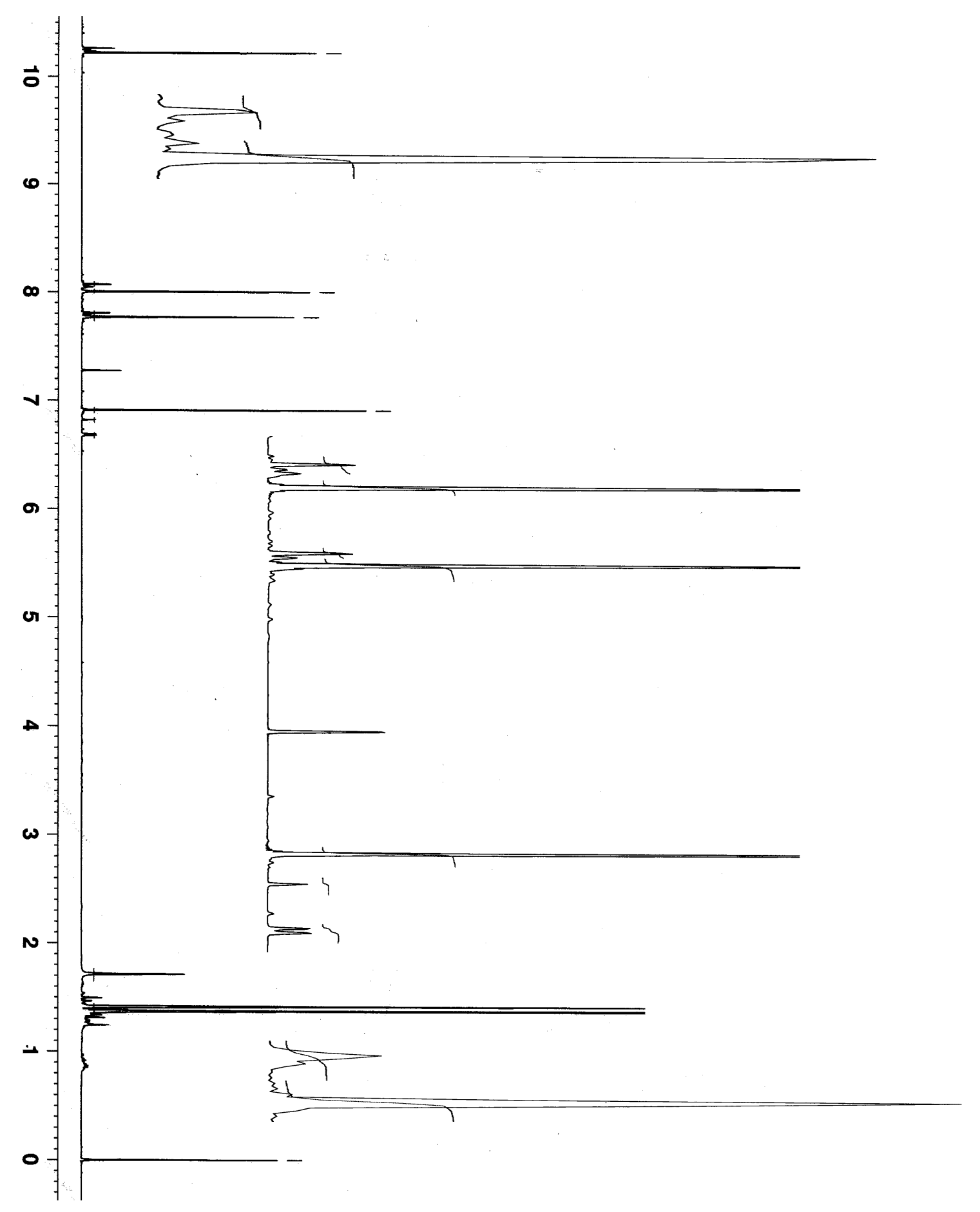


Figure S.22. ${ }^{13} \mathrm{C}$ NMR spectrum of compound 34

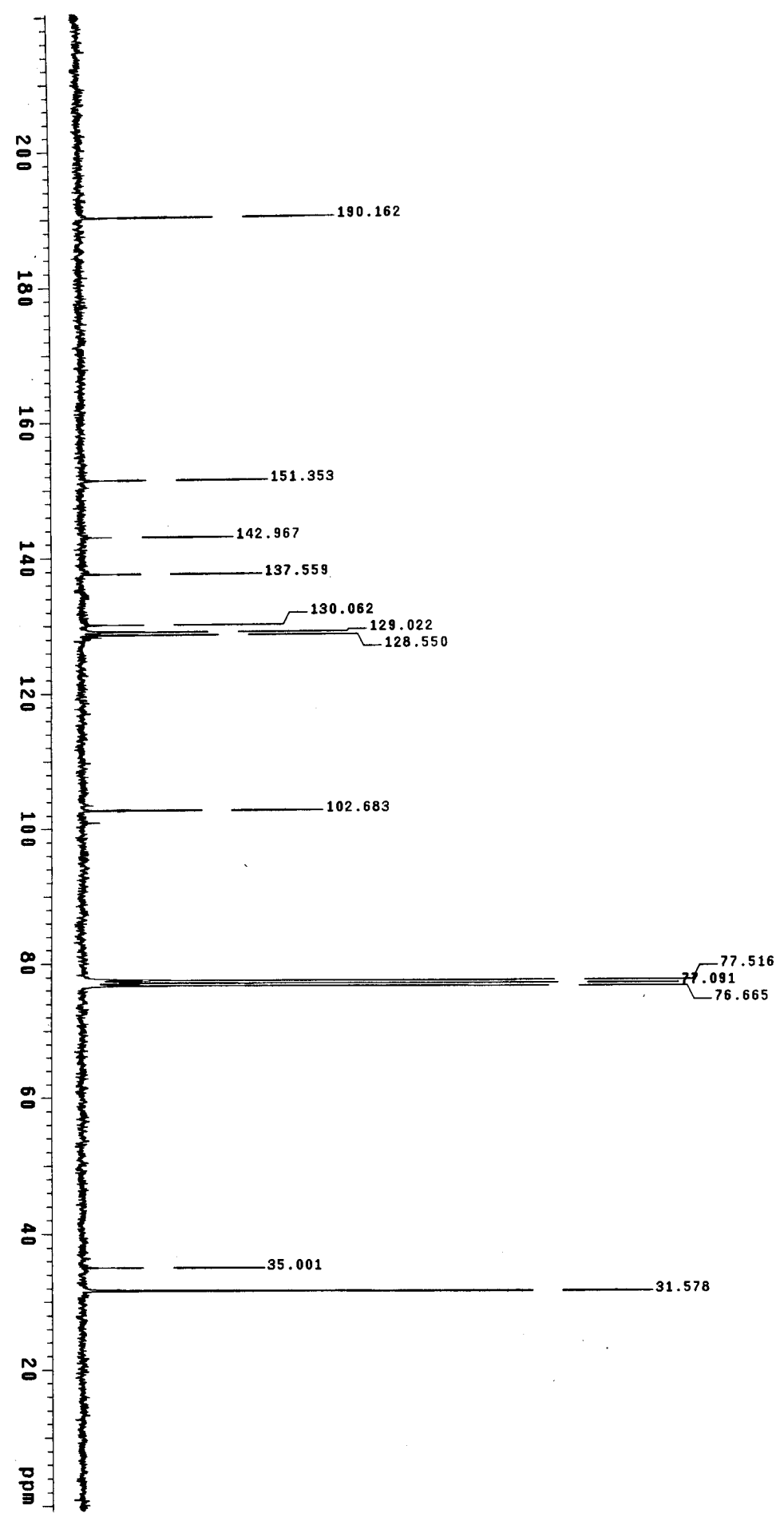


Figure S.23. ${ }^{13} \mathrm{C}$ NMR spectrum of compound 34 with longer acquisition period

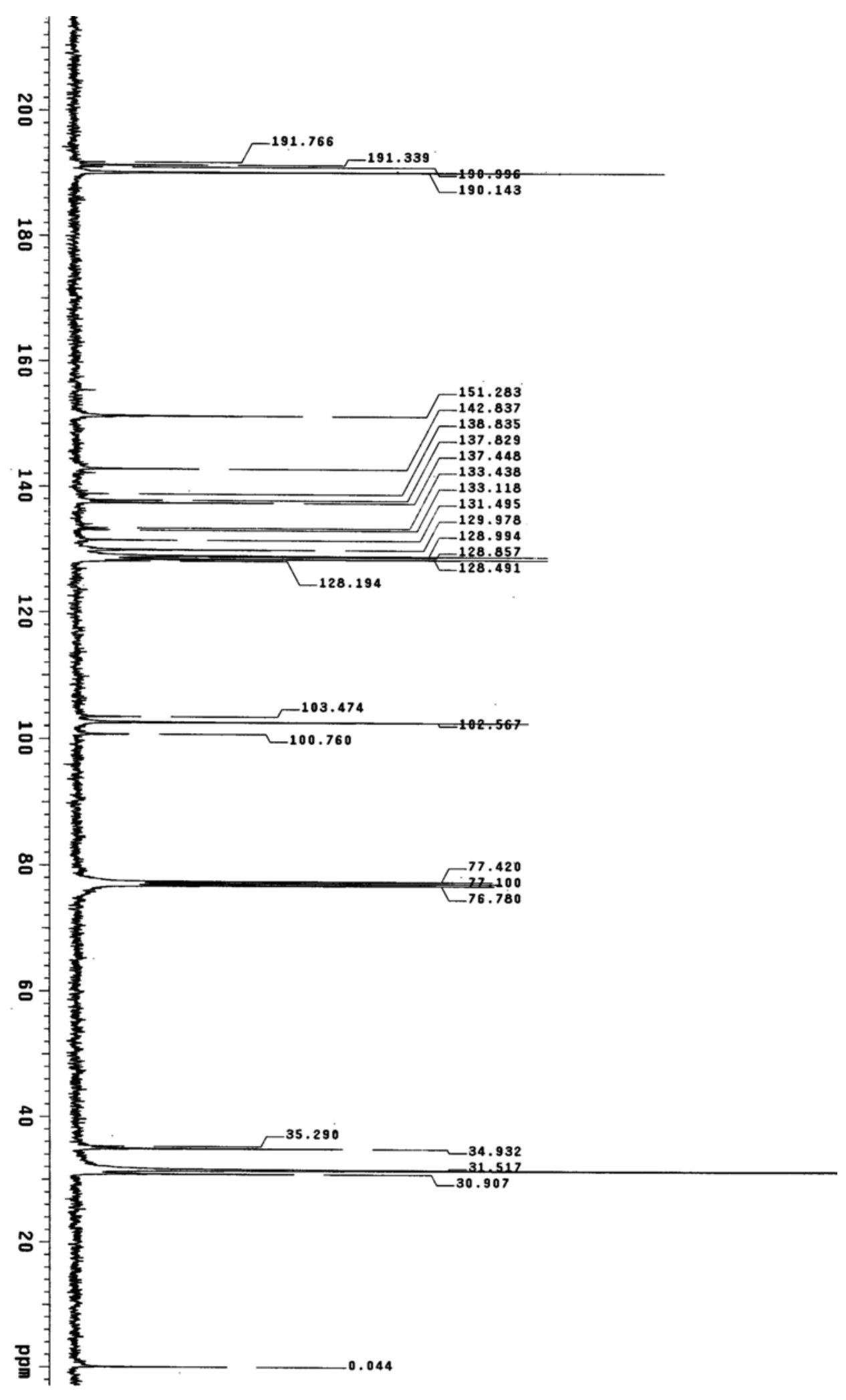


Figure S.24. ${ }^{77}$ Se NMR spectrum of compound 34

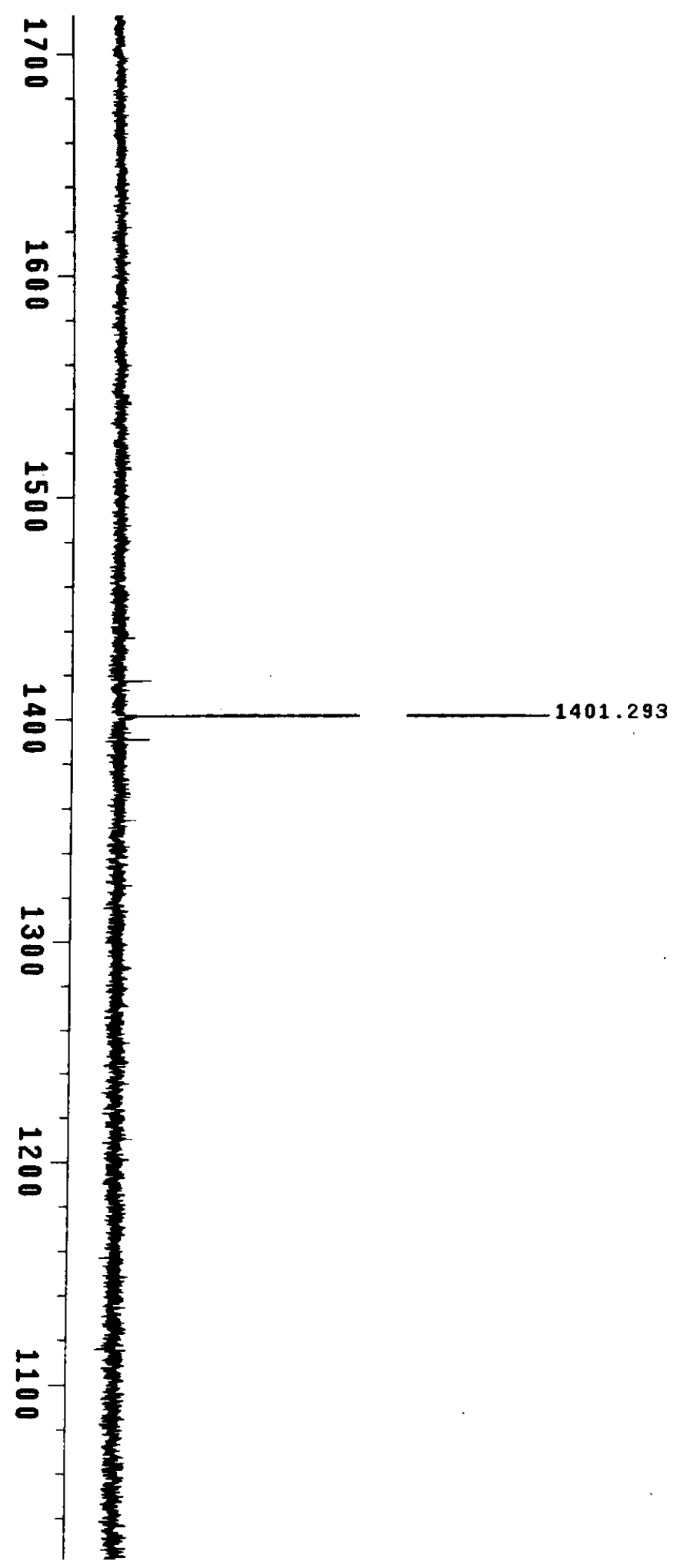


The B3LYP/6-31G* optimized geometries and total energies for various structures.

\begin{tabular}{|lrrr|l|lrr|}
\hline $\mathbf{4 7 a}($ anti,anti) $\mathbf{E t}=\mathbf{- 5 2 9 6 . 9 9 2 6 0 3}$ & \multicolumn{4}{|l|}{$\mathbf{4 7} \mathbf{b}($ syn,anti) $\mathbf{E t}=\mathbf{- 5 2 9 6 . 9 8 8 4 5 9}$} \\
\hline Se & 0.831775 & -0.157794 & 1.215786 & $\mathrm{C}$ & -1.187954 & 1.601351 & -0.084947 \\
$\mathrm{C}$ & -0.902258 & 0.057226 & 0.345679 & $\mathrm{C}$ & -0.942138 & 0.251406 & 0.262760 \\
$\mathrm{C}$ & -1.694027 & -1.070915 & 0.053458 & $\mathrm{C}$ & -1.946748 & -0.707401 & 0.026604 \\
$\mathrm{C}$ & -2.947607 & -0.897803 & -0.557285 & $\mathrm{C}$ & -3.175410 & -0.320213 & -0.537213 \\
$\mathrm{C}$ & -3.412144 & 0.369202 & -0.890011 & $\mathrm{C}$ & -3.419295 & 1.002761 & -0.874831 \\
$\mathrm{C}$ & -2.624793 & 1.482173 & -0.604663 & $\mathrm{C}$ & -2.424649 & 1.951970 & -0.644428 \\
$\mathrm{C}$ & -1.377943 & 1.342339 & 0.020031 & $\mathrm{Se}$ & 0.697479 & -0.271848 & 1.162346 \\
$\mathrm{C}$ & -1.263509 & -2.471528 & 0.333685 & $\mathrm{Se}$ & 2.201939 & -0.036483 & -0.601105 \\
$\mathrm{O}$ & -1.960178 & -3.442634 & 0.104470 & $\mathrm{C}$ & 2.033442 & -1.849894 & -1.366428 \\
$\mathrm{H}$ & -0.251860 & -2.582879 & 0.762604 & $\mathrm{C}$ & -1.769881 & -2.164593 & 0.305312 \\
$\mathrm{C}$ & -0.619730 & 2.592982 & 0.321342 & $\mathrm{O}$ & -2.657360 & -2.982949 & 0.150244 \\
$\mathrm{O}$ & -0.970413 & 3.691783 & -0.063822 & $\mathrm{C}$ & -0.240187 & 2.734138 & 0.109023 \\
$\mathrm{H}$ & 0.283077 & 2.465383 & 0.943875 & $\mathrm{O}$ & 0.928929 & 2.673601 & 0.429295 \\
$\mathrm{H}$ & -3.530292 & -1.789946 & -0.766533 & $\mathrm{H}$ & -0.765979 & -2.464895 & 0.651590 \\
$\mathrm{H}$ & -4.380078 & 0.490761 & -1.367375 & $\mathrm{H}$ & -0.713782 & 3.722828 & -0.081855 \\
$\mathrm{H}$ & -2.954465 & 2.487896 & -0.848173 & $\mathrm{H}$ & -3.917773 & -1.095590 & -0.701066 \\
$\mathrm{Se}$ & 2.225801 & 0.249048 & -0.608606 & $\mathrm{H}$ & -4.370092 & 1.296912 & -1.309027 \\
$\mathrm{C}$ & 2.152060 & -1.520399 & -1.479810 & $\mathrm{H}$ & -2.603839 & 2.994982 & -0.897618 \\
$\mathrm{H}$ & 2.812553 & -1.455648 & -2.348481 & $\mathrm{H}$ & 2.733617 & -1.892238 & -2.205376 \\
$\mathrm{H}$ & 2.514957 & -2.283899 & -0.792399 & $\mathrm{H}$ & 2.305268 & -2.597050 & -0.621292 \\
$\mathrm{H}$ & 1.132961 & -1.727164 & -1.807397 & $\mathrm{H}$ & 1.016736 & -2.004059 & -1.728975 \\
\hline
\end{tabular}

\begin{tabular}{|lrrr|l|l|l|l|}
\hline $\mathbf{4 7 c}$ & $($ syn,syn $) \mathbf{E t}=\mathbf{- 5 2 9 6 . 9 8 3 8 6 2}$ & $\mathbf{4 8 a}($ anti,anti) & $\mathbf{E t}=\mathbf{- 2 8 9 7 . 5 8 8 4 3 6}$ \\
\hline $\mathrm{C}$ & 2.208004 & 2.105342 & 0.478866 & $\mathrm{Se}$ & 0.026680 & -1.749086 & -0.420129 \\
$\mathrm{C}$ & 3.285597 & 1.372940 & 0.968815 & $\mathrm{O}$ & 3.586969 & 0.875830 & -0.011528 \\
$\mathrm{C}$ & 3.268794 & -0.007254 & 0.813362 & $\mathrm{O}$ & -3.616697 & 0.757881 & -0.013087 \\
$\mathrm{C}$ & 2.172742 & -0.657449 & 0.225455 & $\mathrm{C}$ & -0.004353 & 0.176810 & -0.131189 \\
$\mathrm{C}$ & 1.030453 & 0.075123 & -0.179579 & $\mathrm{C}$ & 1.199773 & 0.903157 & -0.049031 \\
$\mathrm{C}$ & 1.092632 & 1.489324 & -0.110919 & $\mathrm{C}$ & 1.162182 & 2.297563 & 0.106615 \\
$\mathrm{C}$ & 2.286438 & -2.113550 & 0.024238 & $\mathrm{C}$ & -0.050103 & 2.976030 & 0.182680 \\
$\mathrm{O}$ & 1.549219 & -2.773004 & -0.687139 & $\mathrm{C}$ & -1.239499 & 2.257846 & 0.110436 \\
$\mathrm{Se}$ & -0.531463 & -0.889105 & -0.762593 & $\mathrm{C}$ & -1.231550 & 0.862857 & -0.044708 \\
$\mathrm{Se}$ & -2.206537 & 0.295294 & 0.381249 & $\mathrm{C}$ & 0.054420 & -2.279254 & 1.474525 \\
$\mathrm{C}$ & -2.216361 & -0.870481 & 1.980401 & $\mathrm{C}$ & 2.543590 & 0.255494 & -0.096549 \\
$\mathrm{C}$ & 0.150681 & 2.431721 & -0.777131 & $\mathrm{C}$ & -2.553320 & 0.170798 & -0.085186 \\
$\mathrm{H}$ & 3.133805 & -2.593816 & 0.558108 & $\mathrm{H}$ & 2.111388 & 2.821842 & 0.166949 \\
$\mathrm{H}$ & 2.231429 & 3.192274 & 0.525028 & $\mathrm{H}$ & -2.205283 & 2.750626 & 0.173374 \\
$\mathrm{H}$ & 4.132068 & 1.871878 & 1.429827 & $\mathrm{H}$ & 2.533990 & -0.843109 & -0.212224 \\
$\mathrm{H}$ & 4.115456 & -0.606196 & 1.142260 & $\mathrm{H}$ & -2.507147 & -0.928601 & -0.182821 \\
$\mathrm{H}$ & -2.988142 & -0.475503 & 2.646946 & $\mathrm{H}$ & 0.070570 & -3.371358 & 1.488738 \\
$\mathrm{H}$ & -2.461322 & -1.893832 & 1.696947 & $\mathrm{H}$ & -0.843470 & -1.919718 & 1.979422 \\
$\mathrm{H}$ & -1.242634 & -0.822299 & 2.468162 & $\mathrm{H}$ & 0.951614 & -1.893792 & 1.961270 \\
$\mathrm{O}$ & -0.791151 & 2.156373 & -1.491158 & $\mathrm{H}$ & -0.067798 & 4.055559 & 0.301023 \\
$\mathrm{H}$ & 0.432936 & 3.495807 & -0.616239 & & & & \\
\hline
\end{tabular}




\begin{tabular}{|c|c|c|c|c|c|c|c|}
\hline \multicolumn{4}{|c|}{ 48b (syn,anti) $\mathbf{E t}=\mathbf{- 2 8 9 7 . 5 8 3 6 1 4}$} & \multicolumn{4}{|c|}{ 48c $($ syn,syn) $\mathbf{E t}=\mathbf{- 2 8 9 7 . 5 7 7 7 8 5}$} \\
\hline $\mathrm{C}$ & -0.032279 & 1.591005 & -0.045850 & $\mathrm{C}$ & -2.774232 & -0.184057 & 0.213652 \\
\hline $\mathrm{C}$ & 0.193316 & 0.194750 & -0.093092 & $\mathrm{C}$ & -1.511004 & -0.759471 & 0.022715 \\
\hline $\mathrm{C}$ & 1.515847 & -0.279948 & -0.000658 & $\mathrm{C}$ & -0.341362 & 0.050106 & -0.013418 \\
\hline $\mathrm{C}$ & 2.588838 & 0.621772 & 0.106025 & $\mathrm{C}$ & -0.529386 & 1.459141 & -0.015822 \\
\hline $\mathrm{C}$ & 2.368201 & 1.991664 & 0.128669 & $\mathrm{C}$ & -1.812552 & 1.995727 & 0.194644 \\
\hline $\mathrm{C}$ & 1.059912 & 2.465710 & 0.052278 & $\mathrm{C}$ & -2.936346 & 1.191694 & 0.335692 \\
\hline $\mathrm{Se}$ & -1.243642 & -1.055846 & -0.446882 & $\mathrm{C}$ & -1.481061 & -2.223913 & -0.146402 \\
\hline $\mathrm{C}$ & -2.005991 & -1.155815 & 1.361449 & $\mathrm{O}$ & -0.513931 & -2.867643 & -0.511724 \\
\hline $\mathrm{C}$ & 1.851925 & -1.735924 & 0.061741 & $\mathrm{Se}$ & 1.353240 & -0.818580 & -0.016166 \\
\hline $\mathrm{O}$ & 2.995325 & -2.152604 & 0.076297 & $\mathrm{C}$ & 2.455597 & 0.407903 & 1.066217 \\
\hline $\mathrm{C}$ & -1.367896 & 2.244851 & -0.101392 & $\mathrm{H}$ & -1.919392 & 3.078600 & 0.208477 \\
\hline $\mathrm{O}$ & -2.447429 & 1.713760 & 0.062190 & $\mathrm{H}$ & -3.639058 & -0.842059 & 0.267187 \\
\hline $\mathrm{H}$ & 3.589504 & 0.205343 & 0.174249 & $\mathrm{H}$ & -2.447036 & -2.730101 & 0.067583 \\
\hline $\mathrm{H}$ & 0.870241 & 3.537117 & 0.069050 & $\mathrm{H}$ & 3.169166 & -0.248296 & 1.570447 \\
\hline $\mathrm{H}$ & 0.984547 & -2.416301 & 0.113435 & $\mathrm{H}$ & 1.826968 & 0.899743 & 1.810470 \\
\hline $\mathrm{H}$ & -1.307679 & 3.338551 & -0.297457 & $\mathrm{H}$ & 2.963858 & 1.138652 & 0.441905 \\
\hline $\mathrm{H}$ & -2.800477 & -1.903945 & 1.310126 & $\mathrm{H}$ & -3.916946 & 1.626930 & 0.499275 \\
\hline $\mathrm{H}$ & -2.425722 & -0.186220 & 1.626948 & $\mathrm{C}$ & 0.483203 & 2.460386 & -0.427630 \\
\hline $\mathrm{H}$ & -1.241492 & -1.478358 & 2.069308 & $\mathrm{O}$ & 1.578704 & 2.244717 & -0.908760 \\
\hline $\mathrm{H}$ & 3.200519 & 2.684961 & 0.205402 & $\mathrm{H}$ & 0.116937 & 3.506570 & -0.329708 \\
\hline
\end{tabular}

\begin{tabular}{|c|c|c|c|c|c|c|c|}
\hline \multicolumn{4}{|c|}{ 50a $(s y n,-S e M e$ in-plane) $\mathbf{E t}=\mathbf{- 2 7 8 4 . 2 6 7 0 1 5}$} & \multicolumn{4}{|c|}{$\begin{array}{l}\text { 50b (anti, Formyl and }- \text { SeMe in plane) } \mathbf{E t}= \\
\text { 2784.261898 }\end{array}$} \\
\hline $\mathrm{Se}$ & -1.576538 & 0.120250 & -0.000152 & $\mathrm{C}$ & 1.269909 & -0.394575 & 0.000019 \\
\hline $\mathrm{O}$ & -0.350204 & 2.563743 & -0.000170 & $\mathrm{C}$ & 0.067940 & 0.351565 & -0.000051 \\
\hline $\mathrm{C}$ & 0.290841 & -0.277667 & -0.000094 & $\mathrm{C}$ & 0.150377 & 1.749238 & -0.000070 \\
\hline $\mathrm{C}$ & 0.786605 & -1.589657 & -0.000358 & $\mathrm{C}$ & 1.387940 & 2.392421 & -0.000042 \\
\hline $\mathrm{C}$ & 2.157158 & -1.842336 & -0.000232 & $\mathrm{C}$ & 2.575693 & 1.656881 & 0.000001 \\
\hline $\mathrm{C}$ & 3.083178 & -0.794733 & 0.000099 & $\mathrm{C}$ & 2.505953 & 0.270352 & 0.000030 \\
\hline $\mathrm{C}$ & 2.609120 & 0.509462 & 0.000267 & $\mathrm{Se}$ & -1.625692 & -0.563670 & -0.000112 \\
\hline $\mathrm{C}$ & 1.231748 & 0.784281 & 0.000145 & $\mathrm{C}$ & -2.865316 & 0.958410 & 0.000323 \\
\hline $\mathrm{C}$ & -2.293160 & -1.719581 & 0.000618 & $\mathrm{C}$ & 1.288169 & -1.875758 & 0.000094 \\
\hline $\mathrm{C}$ & 0.812643 & 2.187563 & 0.000202 & $\mathrm{O}$ & 2.302472 & -2.548702 & 0.000090 \\
\hline $\mathrm{H}$ & 2.503645 & -2.872715 & -0.000417 & $\mathrm{H}$ & 1.418913 & 3.479023 & -0.000063 \\
\hline $\mathrm{H}$ & 3.304590 & 1.346356 & 0.000489 & $\mathrm{H}$ & 3.401779 & -0.343067 & 0.000072 \\
\hline $\mathrm{H}$ & 1.641562 & 2.926842 & 0.000577 & $\mathrm{H}$ & 0.295654 & -2.373668 & 0.000163 \\
\hline $\mathrm{H}$ & -3.378253 & -1.592025 & 0.001900 & $\mathrm{H}$ & -3.859628 & 0.507196 & 0.000461 \\
\hline $\mathrm{H}$ & -1.994873 & -2.260444 & 0.900538 & $\mathrm{H}$ & -2.738113 & 1.559164 & -0.901068 \\
\hline $\mathrm{H}$ & -1.996942 & -2.260447 & -0.899984 & $\mathrm{H}$ & -2.737772 & 1.558932 & 0.901824 \\
\hline $\mathrm{H}$ & 4.149632 & -0.997878 & 0.000205 & $\mathrm{H}$ & 3.537309 & 2.161300 & 0.000013 \\
\hline $\mathrm{H}$ & 0.105771 & -2.432128 & -0.000657 & $\mathrm{H}$ & -0.748387 & 2.354320 & -0.000126 \\
\hline
\end{tabular}




\begin{tabular}{|c|c|c|c|c|c|c|c|}
\hline \multicolumn{4}{|c|}{$\begin{array}{l}\text { 51a }(\operatorname{syn},- \text { SeSe in-plane with formyl) } \mathbf{E t}=\text { - } \\
\text { 5183.678633 }\end{array}$} & \multicolumn{4}{|c|}{$\begin{array}{l}\text { 51b (anti, }- \text { SeSe in-plane with formyl) } \mathbf{E t}=- \\
\text { 5183.671031 }\end{array}$} \\
\hline $\mathrm{C}$ & -3.203706 & 1.978623 & 0.010498 & $\mathrm{C}$ & -1.639317 & 2.557940 & -0.163164 \\
\hline $\mathrm{C}$ & -3.400201 & 0.610574 & 0.130609 & $\mathrm{C}$ & -2.969912 & 2.155474 & -0.012389 \\
\hline $\mathrm{C}$ & -2.312180 & -0.278334 & 0.087929 & $\mathrm{C}$ & -3.257588 & 0.801690 & 0.093862 \\
\hline $\mathrm{C}$ & -0.995310 & 0.213680 & -0.076470 & $\mathrm{C}$ & -2.234539 & -0.160252 & 0.052354 \\
\hline $\mathrm{C}$ & -0.810450 & 1.593312 & -0.198995 & $\mathrm{C}$ & -0.894060 & 0.255486 & -0.100301 \\
\hline $\mathrm{C}$ & -1.899962 & 2.461031 & -0.155972 & $\mathrm{C}$ & -0.610761 & 1.617654 & -0.207330 \\
\hline $\mathrm{C}$ & -2.553103 & -1.711322 & 0.213661 & $\mathrm{C}$ & -2.612097 & -1.586059 & 0.174576 \\
\hline $\mathrm{O}$ & -1.660197 & -2.550214 & 0.178761 & $\mathrm{O}$ & -3.752300 & -1.988694 & 0.306134 \\
\hline $\mathrm{Se}$ & 0.464057 & -1.058041 & -0.122687 & $\mathrm{Se}$ & 0.524408 & -1.086916 & -0.164242 \\
\hline $\mathrm{Se}$ & 2.307405 & 0.390937 & -0.361847 & $\mathrm{Se}$ & 2.450863 & 0.207778 & -0.324467 \\
\hline $\mathrm{C}$ & 2.609421 & 0.808641 & 1.546725 & $\mathrm{C}$ & 2.750228 & 0.572589 & 1.594888 \\
\hline $\mathrm{H}$ & 0.195396 & 1.979761 & -0.335217 & $\mathrm{H}$ & 0.419697 & 1.937892 & -0.330141 \\
\hline $\mathrm{H}$ & -3.608618 & -2.023212 & 0.345880 & $\mathrm{H}$ & -1.769420 & -2.311022 & 0.141212 \\
\hline $\mathrm{H}$ & -1.726868 & 3.529558 & -0.255865 & $\mathrm{H}$ & -1.394886 & 3.613527 & -0.250358 \\
\hline $\mathrm{H}$ & -4.045472 & 2.663546 & 0.043352 & $\mathrm{H}$ & -3.767508 & 2.891738 & 0.020568 \\
\hline $\mathrm{H}$ & -4.402021 & 0.205958 & 0.258290 & $\mathrm{H}$ & -4.276280 & 0.445144 & 0.211695 \\
\hline $\mathrm{H}$ & 3.462246 & 1.491161 & 1.589238 & $\mathrm{H}$ & 3.626533 & 1.224083 & 1.652443 \\
\hline $\mathrm{H}$ & 1.726165 & 1.290085 & 1.967833 & $\mathrm{H}$ & 1.880284 & 1.077436 & 2.014910 \\
\hline $\mathrm{H}$ & 2.843992 & -0.110822 & 2.082670 & $\mathrm{H}$ & 2.949064 & -0.365661 & 2.111727 \\
\hline
\end{tabular}


Computed Natural Charges using Natural Population Analysis at the NPA/B3LYP/631G*//B3LYP/6-31G* level

\begin{tabular}{|c|c|}
\hline 47a $($ anti,anti) $\mathbf{E t}=\mathbf{- 5 2 9 6 . 9 9 2 6 0 3}$ & 47b $($ syn,anti $) \mathbf{E t}=\mathbf{- 5 2 9 6 . 9 8 8 4 5 9}$ \\
\hline Natural & Natural \\
\hline Atom No Charge & Atom No Charge \\
\hline $\mathrm{Se} \quad 1 \quad 0.16226$ & C $\quad 1-0.17065$ \\
\hline C $\quad 2 \quad-0.16412$ & C $\quad 2-0.16042$ \\
\hline C $3-0.17785$ & C $3-0.17306$ \\
\hline C $4 \quad-0.15504$ & C $4-0.15294$ \\
\hline C $\quad 5 \quad-0.23001$ & C $\quad 5 \quad-0.23387$ \\
\hline C $\quad 6 \quad-0.15329$ & C $\quad 6 \quad-0.16826$ \\
\hline C $7-0.17041$ & Se $7 \quad 0.19428$ \\
\hline C $8 \quad 0.38665$ & Se $\quad 8 \quad 0.17630$ \\
\hline O $\quad 9 \quad-0.52450$ & C $\quad 9 \quad-0.86707$ \\
\hline H $\quad 10 \quad 0.16571$ & C $10 \quad 0.38758$ \\
\hline C 110.39286 & O $111-0.52628$ \\
\hline O $\quad 12-0.52190$ & C $\quad 12 \quad 0.39640$ \\
\hline H $13 \quad 0.16592$ & O $\quad 13 \quad-0.51331$ \\
\hline H $14 \quad 0.26298$ & H $14 \quad 0.16986$ \\
\hline H $\quad 15 \quad 0.24964$ & H $15 \quad 0.14514$ \\
\hline H $16 \quad 0.26339$ & H $16 \quad 0.26346$ \\
\hline Se $17 \quad 0.15594$ & H $17 \quad 0.24908$ \\
\hline C $\quad 18-0.86034$ & H $18 \quad 0.24289$ \\
\hline H $19 \quad 0.25436$ & H $19 \quad 0.24999$ \\
\hline H $20 \quad 0.25116$ & H $20 \quad 0.24819$ \\
\hline H $21 \quad 0.24660$ & H $21 \quad 0.24268$ \\
\hline
\end{tabular}




\begin{tabular}{|c|c|}
\hline $47 \mathbf{c}(s y n, s y n) \mathbf{E t}=\mathbf{- 5 2 9 6 . 9 8 3 8 6 2}$ & 48a $($ anti,anti) $\mathbf{E t}=\mathbf{- 2 8 9 7 . 5 8 8 4 3 6}$ \\
\hline Natural & Natural \\
\hline Atom No Charge & Atom No Charge \\
\hline C $\quad 1 \quad-0.16375$ & $\begin{array}{lll}\text { Se } & 1 & 0.32219\end{array}$ \\
\hline C $\quad 2 \quad-0.24696$ & $\begin{array}{lll}\mathrm{O} & 2 & -0.52513\end{array}$ \\
\hline C $3 \quad-0.16407$ & O $3 \quad-0.52514$ \\
\hline C $\quad 4 \quad-0.18016$ & $\begin{array}{lll}C & 4 & -0.16494\end{array}$ \\
\hline C $\quad 5 \quad-0.15608$ & C $\quad 5 \quad-0.17410$ \\
\hline C $\quad 6 \quad-0.18094$ & C $\quad 6 \quad-0.15486$ \\
\hline C $7 \quad 0.39006$ & C $7 \quad-0.23032$ \\
\hline $\begin{array}{lll}\mathrm{O} & 8 & -0.51411\end{array}$ & C $8 \quad-0.15486$ \\
\hline Se $\quad 9 \quad 0.29431$ & C $\quad 9 \quad-0.17413$ \\
\hline Se $\quad 10 \quad 0.13520$ & C $\quad 10 \quad-0.85361$ \\
\hline C $\quad 11-0.86233$ & C $11 \quad 0.38932$ \\
\hline C $12 \quad 0.39837$ & C $12 \quad 0.38944$ \\
\hline $\begin{array}{lll}\mathrm{H} & 13 & 0.15363\end{array}$ & Н $13 \quad 0.26262$ \\
\hline $\begin{array}{lll}\text { H } & 14 & 0.24262\end{array}$ & $\begin{array}{lll}\text { H } & 14 & 0.26262\end{array}$ \\
\hline $\begin{array}{lll}\text { H } & 15 & 0.24790\end{array}$ & $\begin{array}{lll}\text { H } & 15 & 0.16711\end{array}$ \\
\hline $\begin{array}{lll}\mathrm{H} & 16 & 0.24305\end{array}$ & Н $16 \quad 0.16712$ \\
\hline H $17 \quad 0.24644$ & Н $17 \quad 0.25570$ \\
\hline $\begin{array}{lll}\text { H } & 18 & 0.24728\end{array}$ & Н $18 \quad 0.24597$ \\
\hline H $19 \quad 0.24216$ & H $19 \quad 0.24583$ \\
\hline $\begin{array}{lll}\mathrm{O} & 20 & -0.51820\end{array}$ & H $\quad 20 \quad 0.24916$ \\
\hline H 210.14556 & \\
\hline
\end{tabular}




\begin{tabular}{|c|c|}
\hline 48b $($ syn,anti) $\mathbf{E t}=\mathbf{- 2 8 9 7 . 5 8 3 6 1 4}$ & 48c $($ syn,syn) $\mathbf{E t}=-\mathbf{2 8 9 7 . 5 7 7 7 8 5}$ \\
\hline Natural & Natural \\
\hline Atom No Charge & Atom No Charge \\
\hline 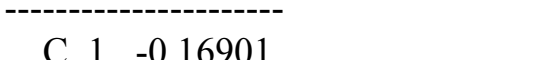 & $C \quad 1 \quad-016343$ \\
\hline $\begin{array}{lll}C & 1 & -0.16901 \\
C & 2 & -0.17286\end{array}$ & $\begin{array}{lll}C & 1 & -0.16343 \\
C & 2\end{array}$ \\
\hline 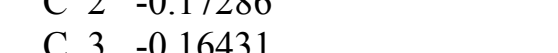 & $C \quad 2 \quad-0.181 / 9$ \\
\hline С $3-0.16431$ & С $3-0.16730$ \\
\hline C $4 \quad-0.15539$ & $\begin{array}{lll}\text { C } & 4 & -0.19158\end{array}$ \\
\hline C $5-0.23255$ & C $\quad 5 \quad-0.15991$ \\
\hline С $6 \quad-0.17239$ & C $\quad 6 \quad-0.25455$ \\
\hline Se $7 \quad 0.35498$ & C $7 \quad 0.38855$ \\
\hline C $8 \quad-0.85268$ & $\begin{array}{lll}O & 8 & -0.51921\end{array}$ \\
\hline C 90.38848 & $\begin{array}{lll}\text { Se } & 9 & 0.49196\end{array}$ \\
\hline O $10-0.52849$ & C $\quad 10 \quad-0.87275$ \\
\hline C $11 \quad 0.39380$ & $\begin{array}{lll}\text { H } & 11 & 0.24089\end{array}$ \\
\hline O $12-0.51569$ & H $12 \quad 0.24260$ \\
\hline H $13 \quad 0.26352$ & H $13 \quad 0.15003$ \\
\hline H $14 \quad 0.24268$ & H $14 \quad 0.24845$ \\
\hline H $15 \quad 0.17308$ & $\begin{array}{lll}\mathrm{H} & 15 & 0.24219\end{array}$ \\
\hline H $16 \quad 0.14631$ & H $16 \quad 0.26176$ \\
\hline Н $17 \quad 0.25255$ & Н $17 \quad 0.24693$ \\
\hline H $18 \quad 0.25765$ & C $\quad 18 \quad 0.38280$ \\
\hline H $19 \quad 0.24145$ & $\begin{array}{lll}\text { O } & 19 & -0.52971\end{array}$ \\
\hline H $20 \quad 0.24887$ & H $20 \quad 0.14409$ \\
\hline
\end{tabular}

\begin{tabular}{|c|c|}
\hline 50a (syn,-SeMe in-plane) $\mathbf{E t}=\mathbf{- 2 7 8 4 . 2 6 7 0 1 5}$ & $\begin{array}{l}\text { 50b (anti, Formyl and }- \text { SeMe in plane) } \mathbf{E t}=- \\
\text { 2784.261898 }\end{array}$ \\
\hline Natural & Natural \\
\hline Atom No Charge & Atom No Charge \\
\hline C $\quad 1 \quad-0.19138$ & C $\quad 1 \quad-0.19138$ \\
\hline C $\quad 2 \quad-0.18648$ & $\begin{array}{lll}\text { C } & 2 & -0.18648\end{array}$ \\
\hline C $3-0.26092$ & C $3-0.26092$ \\
\hline C $\quad 4 \quad-0.19283$ & C $\quad 4 \quad-0.19283$ \\
\hline $\begin{array}{lll}\text { C } & 5 & -0.25078\end{array}$ & $\begin{array}{lll}\text { C } & 5 & -0.25078\end{array}$ \\
\hline C $\quad 6 \quad-0.16744$ & $\begin{array}{lll}\text { C } & 6 & -0.16744\end{array}$ \\
\hline Se $7 \quad 0.36954$ & Se $7 \quad 0.36954$ \\
\hline C $\quad 8 \quad-0.87349$ & C $\quad 8 \quad-0.87349$ \\
\hline $\begin{array}{lll}\text { C } & 9 & 0.39348\end{array}$ & $\begin{array}{lll}\text { C } & 9 & 0.39348\end{array}$ \\
\hline O $\quad 10 \quad-0.52814$ & O $\quad 10 \quad-0.52814$ \\
\hline Н $11 \quad 0.24098$ & Н $11 \quad 0.24098$ \\
\hline H $12 \quad 0.25909$ & Н $12 \quad 0.25909$ \\
\hline Н $13 \quad 0.15086$ & Н $13 \quad 0.15086$ \\
\hline H $14 \quad 0.26195$ & H $\quad 14 \quad 0.26195$ \\
\hline H $\quad 15 \quad 0.24812$ & Н $15 \quad 0.24812$ \\
\hline Н $16 \quad 0.24813$ & Н $16 \quad 0.24813$ \\
\hline H $17 \quad 0.24280$ & H $17 \quad 0.24280$ \\
\hline H $\quad 18 \quad 0.23651$ & H $\quad 18 \quad 0.23651$ \\
\hline
\end{tabular}




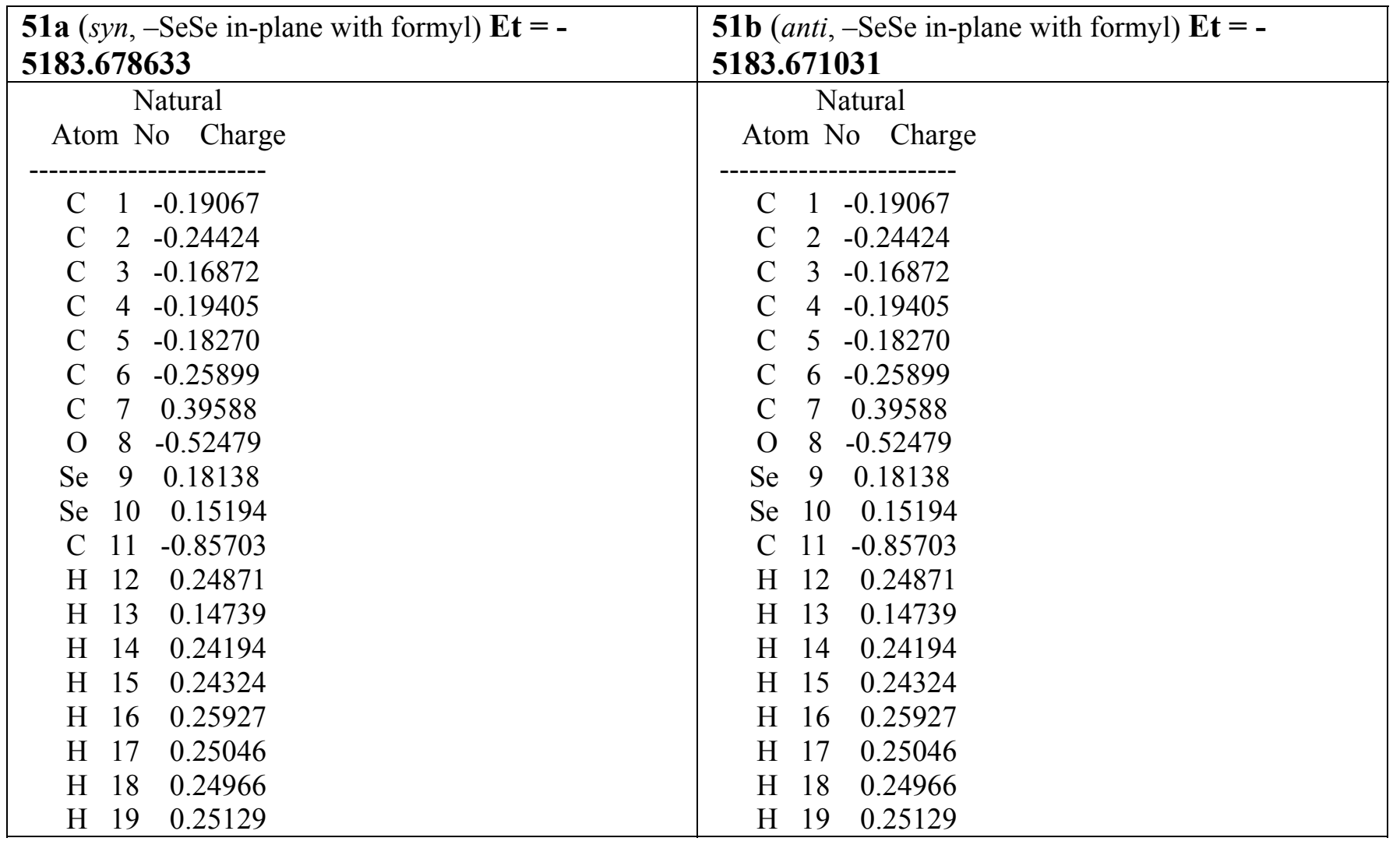


Details of lone-pair delocalizations from oxygen and selenium computed at the NBO/B3LYP/631G*//B3LYP/6-31G* level. Atom numbering are based on the order given earlier in the optimized Cartesian coordinates (pages S27 to S29).

\begin{tabular}{|c|c|c|c|c|c|c|c|}
\hline \multicolumn{8}{|c|}{$47 \mathbf{a}($ anti, anti) $\mathbf{E t}=\mathbf{- 5 2 9 6 . 9 9 2 6 0 3}$} \\
\hline \multicolumn{8}{|c|}{ 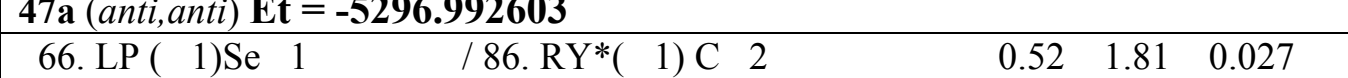 } \\
\hline 66. LP ( & 1) $\mathrm{S}$ & 1 & /218. BD*( & 1) $\mathrm{C} 2-\mathrm{C} 3$ & 0.60 & 1.27 & 0.025 \\
\hline 66. LP ( & 1) $\mathrm{S}$ & 1 & /220. BD*( & 1) $\mathrm{C} \quad 2-\mathrm{C} \quad 7$ & 1.67 & 1.27 & 0.041 \\
\hline 66. LP ( & 1) $\mathrm{S}$ & 1 & /234. BD*( & 1) $\mathrm{C} \quad 8-\mathrm{H} 10$ & $0.67 \quad 1$ & 1.150 & 0.025 \\
\hline 67. LP ( & 2) S & 1 & /191. RY*( & 1)Se 17 & 1.36 & 0.790 & 0.030 \\
\hline 67. LP ( & 2) $\mathrm{S}$ & 1 & /218. BD*( & 1) $\mathrm{C} 2-\mathrm{C} 3$ & 3.23 & 0.80 & 0.046 \\
\hline 67. LP ( & 2) $\mathrm{S}$ & 1 & /219. BD*( & 2) $\mathrm{C} \quad 2-\mathrm{C} \quad 3$ & 1.15 & 0.26 & 0.017 \\
\hline 67. LP ( & 2) $\mathrm{S}$ & 1 & /220. BD*( & 1) $\mathrm{C} \quad 2-\mathrm{C} \quad 7$ & 2.38 & 0.80 & 0.039 \\
\hline 67. LP ( & 2) $\mathrm{S}$ & 1 & /234. BD*( & 1) $\mathrm{C} \quad 8-\mathrm{H} 10$ & 2.13 & 0.67 & $0.034(*)$ \\
\hline 67. LP ( & 2)S & 1 & /235. BD*( & 1) $\mathrm{C} 11-\mathrm{O} 12$ & 0.52 & 0.85 & 0.019 \\
\hline 67. LP ( & 2) $\mathrm{S}$ & 1 & /237. BD*( & 1) $\mathrm{C} 11-\mathrm{H} 13$ & 2.52 & 0.67 & $0.037(*)$ \\
\hline 67. LP ( & 2) $\mathrm{S}$ & 1 & /238. BD*( & 1)Se 17 - C 18 & 4.28 & 0.34 & 0.034 \\
\hline 67. LP ( & 2) $\mathrm{S}$ & 1 & /239. BD*( & 1) $\mathrm{C} 18-\mathrm{H} 19$ & 0.51 & 0.69 & 0.017 \\
\hline 68. LP ( & 1) ( & 9 & /146. RY*( & 1) $\mathrm{C} 8$ & 11.96 & 1.49 & 0.119 \\
\hline 68. LP ( & 1) $\mathrm{C}$ & 9 & /222. BD*( & 1) $\mathrm{C} \quad 3-\mathrm{C} \quad 8$ & 1.66 & 1.12 & 0.039 \\
\hline 68. LP ( & 1) $\mathrm{C}$ & 9 & /234. BD*( & 1) $\mathrm{C} \quad 8-\mathrm{H} \quad 10$ & 1.10 & 1.11 & 0.032 \\
\hline 69. LP ( & 2) $\mathrm{C}$ & 9 & /147. RY*( & 2) $\mathrm{C} \quad 8$ & 2.14 & 2.23 & 0.063 \\
\hline 69. LP ( & 2) $\mathrm{C}$ & 9 & /150. RY*( & 5) $\mathrm{C} \quad 8$ & 0.85 & 1.96 & 0.037 \\
\hline 69. LP ( & 2) $\mathrm{C}$ & 9 & /166. RY*( & 1) $\mathrm{H} 10$ & 0.67 & 0.95 & 0.023 \\
\hline 69. LP ( & 2) ( & 9 & /222. BD*( & 1) $C \quad 3-C \quad 8$ & 20.23 & 0.69 & 0.107 \\
\hline 69. LP ( & 2) $\mathrm{C}$ & & /225. BD*( & 1) $\mathrm{C} \quad 4-\mathrm{H} 14$ & 0.52 & 0.74 & 0.018 \\
\hline 69. LP ( & 2) ( & 9 & /234. BD*( & 1) $\mathrm{C} 8-\mathrm{H} 10$ & 20.20 & 0.68 & 0.106 \\
\hline 70. LP ( & 1) ( & 12 & /167. RY* & 1) C 11 & 11.96 & 1.49 & 0.119 \\
\hline 70. LP ( & 1) $\mathrm{C}$ & 12 & /231. BD*( & 1) $\mathrm{C} 7$ - C 11 & 1.62 & 1.12 & 0.038 \\
\hline 70. LP ( & 1) $\mathrm{C}$ & 12 & /237. BD*( & 1) $\mathrm{C} 11-\mathrm{H} 13$ & 1.11 & 1.11 & 0.032 \\
\hline 71. LP ( & 2) $\mathrm{C}$ & 12 & /168. RY*( & 2) C 11 & 2.13 & 2.21 & 0.063 \\
\hline 71. LP ( & 2) $\mathrm{C}$ & 12 & /171. RY*( & 5) C 11 & 0.90 & 1.69 & 0.036 \\
\hline 71. LP ( & 2) $\mathrm{C}$ & 12 & /187. RY*( & 1) $\mathrm{H} 13$ & 0.67 & 0.94 & 0.023 \\
\hline 71. LP ( & 2) ( & 12 & /231. BD*( & 1) $\mathrm{C} \quad 7$ - C 11 & 20.38 & 0.69 & 0.107 \\
\hline 71. LP ( & 2) ( & 12 & /237. BD*( & 1) C 11 - H 13 & 20.20 & 0.68 & 0.106 \\
\hline 72. LP ( & 1) $\mathrm{S}$ & 17 & /240. BD*( & 1) $\mathrm{C} 18-\mathrm{H} 20$ & 0.69 & 1.16 & 0.025 \\
\hline 73. LP ( & 2)S & 17 & / 74. RY*( & 1)Se 1 & 1.62 & 0.85 & 0.033 \\
\hline 73. LP ( & 2) $\mathrm{S}$ & 17 & /216. BD*( & 1) $\mathrm{Se} 1-\mathrm{C} 2$ & 5.02 & 0.34 & 0.037 \\
\hline 73. LP ( & 2) $\mathrm{S}$ & 17 & /219. BD*( & 2) $\mathrm{C} \quad 2$ - $\mathrm{C} 3$ & 0.98 & 0.25 & 0.015 \\
\hline 73. LP ( & 2)S & 17 & /240. BD*( & 1) $\mathrm{C} 18-\mathrm{H} 20$ & 1.93 & 0.70 & 0.033 \\
\hline 73. LP ( & 2) $\mathrm{S}$ & 17 & /241. BD*( & 1) $\mathrm{C} 18-\mathrm{H} 21$ & 2.74 & 0.70 & 0.040 \\
\hline
\end{tabular}




\begin{tabular}{|c|c|c|c|c|c|c|c|}
\hline \multicolumn{8}{|c|}{ 47b $($ syn,anti) $\mathbf{E t}=\mathbf{- 5 2 9 6 . 9 8 8 4 5 9}$} \\
\hline 66. LP ( & 1) $\mathrm{Se}$ & 7 & / 84. RY*( & 1) $\mathrm{C} 2$ & 0.55 & $1.80 \quad 0$ & 0.028 \\
\hline 66. LP ( & 1) $\mathrm{Se}$ & 7 & /216. BD*( & 1) $\mathrm{C} \quad 1-\mathrm{C} 2$ & 1.92 & 1.25 & 0.044 \\
\hline 66. LP ( & 1) $\mathrm{Se}$ & 7 & /220. $\mathrm{BD} *($ & 1) $\mathrm{C} 2-\mathrm{C} 3$ & 0.54 & 1.26 & 0.023 \\
\hline 66. LP ( & 1) $\mathrm{Se}$ & 7 & /238. BD*( & 1) $\mathrm{C} 10-\mathrm{H} 14$ & 0.70 & 1.14 & 0.026 \\
\hline 67. LP ( & 2)Se & 7 & / 85. RY*( & 2) $\mathrm{C} \quad 2$ & 0.52 & 1.02 & 0.021 \\
\hline 67. LP ( & 2)Se & 7 & /146. RY*( & 1)Se 8 & 1.26 & $0.79 \quad c$ & 0.029 \\
\hline 67. LP ( & 2) $\mathrm{Se}$ & 7 & /216. BD*( & 1) $\mathrm{C} \quad 1-\mathrm{C} 2$ & 2.48 & 0.77 & 0.040 \\
\hline 67. LP ( & 2) $\mathrm{Se}$ & 7 & /220. BD*( & 1) $\mathrm{C} \quad 2-\mathrm{C} 3$ & 3.09 & 0.78 & 0.045 \\
\hline 67. LP ( & 2) $\mathrm{Se}$ & 7 & /221. BD*( & 2) $\mathrm{C} \quad 2-\mathrm{C} 3$ & 2.55 & 0.24 & 0.024 \\
\hline 67. LP & 2) $\mathrm{Se}$ & 7 & /232. BD*( & 1) $\mathrm{Se} \quad 8-\mathrm{C} 9$ & 3.96 & $0.33 \quad c$ & 0.033 \\
\hline 67. LP ( & 2) $\mathrm{Se}$ & 7 & /233. $\mathrm{BD} *($ & 1) $\mathrm{C} 9-\mathrm{H} 19$ & 0.64 & $0.68 \quad 0$ & 0.019 \\
\hline 67. LP ( & 2)Se & 7 & /238. BD*( & 1) $\mathrm{C} 10-\mathrm{H} 14$ & 2.16 & $0.66 \quad c$ & $0.034(*)$ \\
\hline 68. LP ( & 1) $\mathrm{Se}$ & 8 & /234. BD*( & 1) $\mathrm{C} 9-\mathrm{H} 20$ & 0.69 & $1.17 \mathrm{C}$ & 0.025 \\
\hline 69. LP ( & 2) $\mathrm{Se}$ & 8 & /134. RY*( & 1)Se 7 & 1.50 & $0.83 \mathrm{c}$ & 0.032 \\
\hline 69. LP ( & 2) $\mathrm{Se}$ & 8 & /221. BD*( & 2) $\mathrm{C} \quad 2-\mathrm{C} \quad 3$ & 1.07 & 0.24 & 0.015 \\
\hline 69. LP ( & 2)Se & 8 & /222. BD*( & 1) $\mathrm{C} \quad 2-\mathrm{Se} \quad 7$ & 5.07 & 0.34 & 0.037 \\
\hline 69. LP ( & 2) $\mathrm{Se}$ & 8 & /234. BD*( & 1) $\mathrm{C} 9-\mathrm{H} 20$ & 2.10 & 0.69 & 0.034 \\
\hline 69. LP ( & 2)Se & 8 & /235. BD*( & 1) $\mathrm{C} 9-\mathrm{H} 21$ & 2.72 & 0.69 & 0.039 \\
\hline 70. LP ( & 1) $\mathrm{O}$ & & /168. RY*( & 1) $\mathrm{C} 10$ & 11.96 & 1.49 & 0.119 \\
\hline 70. LP ( & 1) $\mathrm{O}$ & 11 & /224. BD*( & 1) $\mathrm{C} \quad 3-\mathrm{C} \quad 10$ & 1.63 & 1.12 & 0.039 \\
\hline 70. LP ( & 1) $\mathrm{O}$ & 11 & /238. BD*( & 1) $\mathrm{C} 10-\mathrm{H} 14$ & 1.10 & 1.12 & 0.032 \\
\hline 71. LP ( & 2) $\mathrm{O}$ & 11 & /169. RY*( & 2) $\mathrm{C} 10$ & 2.13 & 2.24 & 0.063 \\
\hline 71. LP ( & 2) $\mathrm{O}$ & 11 & /172. RY*( & 5) $\mathrm{C} 10$ & 0.84 & 1.96 & 0.037 \\
\hline 71. LP ( & 2) $\mathrm{O}$ & & /208. RY*( & 1) $\mathrm{H} 14$ & 0.67 & 0.95 & 0.023 \\
\hline 71. LP ( & 2) $\mathrm{O}$ & & /224. BD*( & ( 1) C 3 -C 10 & 20.35 & 0.69 & 0.107 \\
\hline 71. LP ( & 2) $\mathrm{O}$ & & /227. BD*( & 1) $\mathrm{C} 4-\mathrm{H} 16$ & 0.56 & 0.74 & 0.019 \\
\hline 71. LP ( & 2) 0 & & /238. BD* & ( 1) C $10-\mathrm{H} 14$ & 19.94 & 0.69 & 0.106 \\
\hline 72. LP ( & 1) $\mathrm{O}$ & 13 & /188. RY* & (1) C 12 & 12.29 & 1.48 & 0.120 \\
\hline 72. LP ( & 1) $\mathrm{O}$ & & /219. BD*( & 1) $\mathrm{C} 1$ - $\mathrm{C} 12$ & 2.01 & 1.12 & 0.043 \\
\hline 72. LP ( & 1) $\mathrm{O}$ & & /232. BD*( & 1) $\mathrm{Se} 8-\mathrm{C} 9$ & 0.55 & 0.79 & 0.019 \\
\hline 72. LP ( & 1) $\mathrm{O}$ & 13 & /241. BD*( & 1) $\mathrm{C} 12-\mathrm{H} 15$ & 0.99 & 1.09 & 0.030 \\
\hline 73. LP ( & 2) $\mathrm{O}$ & 13 & /189. RY*( & 2) $\mathrm{C} 12$ & 2.13 & 2.22 & 0.063 \\
\hline 73. LP ( & 2) $\mathrm{O}$ & 13 & /192. RY*( & 5) $\mathrm{C} 12$ & 1.15 & 1.83 & 0.042 \\
\hline 73. LP ( & 2) $\mathrm{O}$ & & /203. RY*( & 6) $\mathrm{O} 13$ & 0.51 & 2.35 & 0.032 \\
\hline 73. LP ( & 2) $\mathrm{O}$ & & /209. RY*( & 1) $\mathrm{H} 15$ & 0.70 & 0.90 & 0.023 \\
\hline 73. LP ( & 2) $\mathrm{O}$ & & 1219. BD* & ( 1) C 1 - C 12 & 19.96 & 0.69 & 0.107 \\
\hline 73. LP ( & 2) $\mathrm{O}$ & & /232. BD*( & 1) $\mathrm{Se} 8$-C 9 & 0.66 & 0.36 & 0.014 \\
\hline 73. LP ( & 2) $\mathrm{O}$ & 13 & /241. BD*( & ( 1) C $12-\mathrm{H} 15$ & 21.69 & 0.67 & 0.109 \\
\hline
\end{tabular}




\begin{tabular}{|c|c|c|c|c|c|}
\hline \multicolumn{6}{|c|}{ 47c $(s y n, s y n) \mathbf{E t}=\mathbf{- 5 2 9 6 . 9 8 3 8 6 2}$} \\
\hline 66. LP ( 1) O 8 & /134. RY*( & 1) $\mathrm{C} 7$ & 11.53 & 1.48 & 0.117 \\
\hline 66. LP ( 1) O 8 & /227. BD*( & 1) $\mathrm{C} \quad 4-\mathrm{C} \quad 7$ & 2.43 & 1.14 & 0.047 \\
\hline 66. LP ( 1) O 8 & /233. BD*( & 1) $\mathrm{C} 7-\mathrm{H} 13$ & 0.56 & 1.10 & 0.022 \\
\hline 66. LP ( 1) O 8 & /234. BD*( & 1)Se 9 -Se 10 & 1.07 & 0.71 & 0.025 \\
\hline 67. LP ( 2) O 8 & $/ 135 . \mathrm{RY} *($ & 2) $\mathrm{C} 7$ & 2.19 & 2.32 & 0.066 \\
\hline 67. LP ( 2) O 8 & /138. RY*( & 5) $\mathrm{C} 7$ & 0.82 & 1.83 & 0.036 \\
\hline 67. LP ( 2) O 8 & /198. RY*( & 1) $\mathrm{H} 13$ & 0.67 & 0.89 & 0.023 \\
\hline 67. LP ( 2) O 8 & /227. BD*( & 1) $\mathrm{C} \quad 4-\mathrm{C} \quad 7$ & 17.13 & 0.72 & 0.101 \\
\hline 67. LP ( 2) O 8 & /233. BD*( & 1) $\mathrm{C} \quad 7-\mathrm{H} 13$ & 21.43 & 0.68 & 0.110 \\
\hline 67. LP ( 2) O 8 & /234. BD*( & 1)Se 9 -Se 10 & 3.77 & 0.29 & 0.030 \\
\hline 68. LP ( 1)Se 9 & /114. RY*( & 1) $\mathrm{C} 5$ & 0.85 & 1.75 & 0.034 \\
\hline 68. LP ( 1)Se 9 & /228. BD*( & 1) $\mathrm{C} 5$ - $\mathrm{C} \quad 6$ & 2.44 & 1.25 & 0.050 \\
\hline 69. LP ( 2)Se 9 & /166. RY*( & 1)Se 10 & 1.16 & 0.78 & 0.028 \\
\hline 69. LP ( 2)Se 9 & /225. BD*( & 1) $\mathrm{C} \quad 4-\mathrm{C} 5$ & 1.57 & 0.76 & 0.032 \\
\hline 69. LP ( 2)Se 9 & /226. BD*( & 2) $\mathrm{C} \quad 4-\mathrm{C} 5$ & 10.10 & 0.23 & 0.046 \\
\hline 69. LP ( 2)Se 9 & /228. BD*( & 1) $\mathrm{C} 5$ - C 6 & 1.17 & 0.76 & 0.027 \\
\hline 69. LP ( 2)Se 9 & /235. BD*( & 1) $\mathrm{Se} 10$ - C 11 & 3.21 & 0.33 & 0.030 \\
\hline 69. LP ( 2)Se 9 & /236. BD*( & 1) $\mathrm{C} 11-\mathrm{H} 17$ & 0.82 & 0.68 & 0.022 \\
\hline 70. LP ( 1)Se 10 & /237. BD*( & 1) $\mathrm{C} 11-\mathrm{H} 18$ & 0.66 & 1.17 & 0.025 \\
\hline 71. LP ( 2)Se 10 & /154. RY*( & 1)Se 9 & 1.01 & 0.85 & 0.026 \\
\hline 71. LP ( 2)Se 10 & /226. BD*( & 2) $\mathrm{C} \quad 4$ - $\mathrm{C} 5$ & 0.51 & 0.23 & 0.010 \\
\hline 71. LP ( 2)Se 10 & /229. BD*( & 1) $\mathrm{C} \quad 5$-Se 9 & 5.32 & 0.34 & 0.038 \\
\hline 71. LP ( 2)Se 10 & /237. BD*( & 1) $\mathrm{C} 11-\mathrm{H} 18$ & 2.20 & 0.69 & 0.035 \\
\hline 71. LP ( 2)Se 10 & /238. BD*( & 1) $\mathrm{C} 11-\mathrm{H} 19$ & 2.56 & 0.69 & 0.038 \\
\hline 72. LP ( 1) O 20 & /188. RY*( & 1) $\mathrm{C} 12$ & 12.46 & 1.48 & 0.121 \\
\hline 72. LP ( 1) O 20 & 1230. $\mathrm{BD}^{*}($ & 1) $\mathrm{C} \quad 6$ - C 12 & 2.05 & 1.12 & 0.043 \\
\hline 72. LP ( 1) O 20 & /235. BD*( & 1)Se 10 - C 11 & 0.78 & 0.80 & 0.022 \\
\hline 72. LP ( 1) O 20 & /241. BD*( & 1) $\mathrm{C} 12$ - H 21 & 0.99 & 1.09 & 0.030 \\
\hline 73. LP ( 2) O 20 & /189. RY*( & 2) $\mathrm{C} 12$ & 2.13 & 2.20 & 0.063 \\
\hline 73. LP ( 2) O 20 & /192. RY*( & 5) C 12 & 1.05 & 1.76 & 0.040 \\
\hline 73. LP ( 2) O 20 & /215. RY*( & 1) $\mathrm{H} 21$ & 0.70 & 0.90 & 0.023 \\
\hline 73. LP ( 2) O 20 & /230. BD*( & 1) $\mathrm{C} \quad 6$-C 12 & 20.09 & 0.69 & 0.107 \\
\hline 73. LP ( 2) O 20 & /235. BD*( & 1)Se 10 - C 11 & 0.63 & 0.37 & 0.014 \\
\hline 73. LP ( 2) O 20 & /241. BD*( & 1) C 12 - H 21 & 21.81 & 0.67 & 0.109 \\
\hline
\end{tabular}




\begin{tabular}{|c|c|c|c|c|c|c|c|}
\hline \\
\hline \multicolumn{8}{|c|}{ 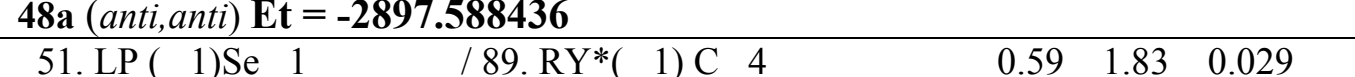 } \\
\hline 51. LP ( & 1) $\mathrm{Se}$ & 1 & /193. BD*( & 1) $\mathrm{C} \quad 4-\mathrm{C} 5$ & 1.07 & 1.24 & 0.033 \\
\hline 51. LP ( & 1) $\mathrm{Se}$ & 1 & $/ 194 . \mathrm{BD}^{*}($ & 1) $\mathrm{C} 4$ - $\mathrm{C} \quad 9$ & 1.03 & 1.24 & 0.032 \\
\hline 51. LP ( & 1) $\mathrm{Se}$ & 1 & $/ 195 . \mathrm{BD} *($ & 2) $\mathrm{C} \quad 4-\mathrm{C} \quad 9$ & 0.67 & 0.70 & 0.021 \\
\hline 51. LP ( & 1) $\mathrm{Se}$ & 1 & /208. BD*( & 1) $\mathrm{C} 10-\mathrm{H} 18$ & 0.54 & 1.14 & 0.022 \\
\hline 51. LP ( & 1) $\mathrm{Se}$ & 1 & $/ 209 . \mathrm{BD} *($ & 1) $\mathrm{C} 10-\mathrm{H} 19$ & 0.56 & 1.14 & 0.023 \\
\hline 52. LP ( & 2) $\mathrm{Se}$ & 1 & / 90. RY*( & 2) $\mathrm{C} \quad 4$ & 0.69 & 1.03 & 0.024 \\
\hline 52. LP ( & 2) $\mathrm{Se}$ & 1 & /189. BD*( & 1) $\mathrm{O} \quad 2$ - $\mathrm{C} 11$ & 0.57 & 0.83 & 0.020 \\
\hline 52. LP ( & 2) $\mathrm{Se}$ & 1 & $/ 191 . \mathrm{BD} *($ & 1) $\mathrm{O} \quad 3-\mathrm{C} 12$ & 0.57 & 0.83 & 0.020 \\
\hline 52. LP ( & 2) $\mathrm{Se}$ & 1 & /193. BD*( & 1) $\mathrm{C} 4-\mathrm{C} 5$ & 3.29 & 0.78 & 0.046 \\
\hline 52. LP ( & 2) $\mathrm{Se}$ & 1 & /194. BD*( & 1) $\mathrm{C} \quad 4-\mathrm{C} 9$ & 3.33 & 0.78 & 0.046 \\
\hline 52. LP ( & 2) $\mathrm{Se}$ & 1 & /208. BD*( & 1) $\mathrm{C} 10-\mathrm{H} 18$ & 2.85 & 0.68 & 0.040 \\
\hline 52. LP ( & 2) $\mathrm{Se}$ & 1 & /209. BD*( & 1) $\mathrm{C} 10-\mathrm{H} 19$ & 2.85 & 0.68 & 0.040 \\
\hline 52. LP ( & 2) $\mathrm{Se}$ & 1 & /210. BD*( & 1) $\mathrm{C} 11-\mathrm{H} 15$ & 2.94 & 0.65 & $0.039(*)$ \\
\hline 52. LP ( & 2) $\mathrm{Se}$ & 1 & $/ 211 . \mathrm{BD} *($ & 1) $\mathrm{C} 12$ - $\mathrm{H} 16$ & 2.92 & 0.65 & $0.039(*)$ \\
\hline 53. LP ( & 1) $\mathrm{C}$ & 2 & /159. RY* & 1) $\mathrm{C} 11$ & 12.00 & 1.49 & 0.119 \\
\hline 53. LP ( & 1) $\mathrm{C}$ & 2 & /198. BD*( & 1) $\mathrm{C} \quad 5$ - $\mathrm{C} 11$ & 1.64 & 1.12 & 0.039 \\
\hline 53. LP ( & 1) $\mathrm{C}$ & 2 & /210. BD*( & 1) $\mathrm{C} 11-\mathrm{H} 15$ & 1.11 & 1.11 & 0.032 \\
\hline 54. LP ( & 2) $\mathrm{C}$ & 2 & /160. RY*( & 2) $\mathrm{C} 11$ & 2.14 & 2.24 & 0.064 \\
\hline 54. LP ( & 2) $\mathrm{C}$ & 2 & /163. RY*( & 5) C 11 & 0.89 & 1.90 & 0.038 \\
\hline 54. LP ( & 2) $\mathrm{C}$ & 2 & /181. RY*( & 1) $\mathrm{H} 15$ & 0.67 & 0.94 & 0.023 \\
\hline 54. LP ( & 2) $\mathrm{C}$ & 2 & /198. BD* & 1) C 5-C 11 & 20.26 & 0.69 & 0.107 \\
\hline 54. LP ( & 2) $\mathrm{C}$ & 2 & $/ 200 . \mathrm{BD}^{*}($ & 1) $\mathrm{C} \quad 6-\mathrm{H} 13$ & 0.51 & 0.74 & 0.018 \\
\hline 54. LP ( & 2) $\mathrm{C}$ & 2 & /210. BD* & 1) $\mathrm{C} 11$ - H 15 & 20.13 & 0.68 & 0.106 \\
\hline 55. LP ( & 1) $\mathrm{C}$ & 3 & /169. RY* & 1) $\mathrm{C} 12$ & 12.00 & 1.49 & 0.119 \\
\hline 55. LP ( & 1) $\mathrm{C}$ & 3 & /206. BD*( & 1) $\mathrm{C} 9$ - $\mathrm{C} 12$ & 1.64 & 1.12 & 0.039 \\
\hline 55. LP ( & 1) $\mathrm{C}$ & 3 & /211. BD*( & 1) $\mathrm{C} 12$ - H 16 & 1.11 & 1.11 & 0.032 \\
\hline 56. LP ( & 2) $\mathrm{C}$ & 3 & /170. RY*( & 2) $\mathrm{C} 12$ & 2.14 & 2.24 & 0.064 \\
\hline 56. LP ( & 2) $\mathrm{C}$ & 3 & /173. RY*( & 5) $\mathrm{C} 12$ & 0.89 & 1.90 & 0.038 \\
\hline 56. LP ( & 2) $\mathrm{C}$ & 3 & /182. RY*( & 1) $\mathrm{H} 16$ & 0.67 & 0.94 & 0.023 \\
\hline 56. LP ( & 2) $\mathrm{C}$ & 3 & /205. BD*( & 1) $\mathrm{C} \quad 8-\mathrm{H} 14$ & 0.51 & 0.74 & 0.018 \\
\hline 56. LP ( & 2) $\mathrm{C}$ & 3 & /206. BD* & 1) $\mathrm{C}$ 9-C 12 & 20.26 & 0.69 & 0.107 \\
\hline 56. LP ( & 2) $\mathrm{C}$ & 3 & /211. BD* & 1) C 12 - H 16 & 20.13 & 0.68 & 0.106 \\
\hline
\end{tabular}




\begin{tabular}{|c|c|c|c|c|}
\hline \multicolumn{5}{|c|}{ 48b $($ syn,anti) $\mathbf{E t}=\mathbf{- 2 8 9 7 . 5 8 3 6 1 4}$} \\
\hline 51. LP ( 1)Se 7 & /67. RY*( 1) C 2 & 0.52 & 1.79 & 0.027 \\
\hline 51. LP ( 1)Se 7 & /187. BD*( 1) C 1 - C 2 & 1.85 & 1.21 & 0.042 \\
\hline 51. LP ( 1)Se 7 & $/ 191 . \mathrm{BD}^{*}(1) \mathrm{C} \quad 2-\mathrm{C} 3$ & 0.52 & 1.22 & 0.023 \\
\hline 51. LP ( 1)Se 7 & /192. BD*( 2) C $2-\mathrm{C} 3$ & 0.90 & 0.68 & 0.024 \\
\hline 51. LP ( 1)Se 7 & /204. BD*( 1) C $8-\mathrm{H} 18$ & 0.70 & 1.15 & 0.025 \\
\hline 51. LP ( 1)Se 7 & /208. BD*( 1) C $9-\mathrm{H} 15$ & 0.60 & 1.11 & 0.023 \\
\hline 52. LP ( 2)Se 7 & / 68. RY*( 2) C 2 & 0.62 & 1.10 & 0.024 \\
\hline 52. LP ( 2)Se 7 & /187. BD*( 1) C 1 - C 2 & 2.98 & 0.75 & 0.043 \\
\hline 52. LP ( 2)Se 7 & /191. BD*( 1) C 2 - C 3 & 3.60 & 0.76 & 0.047 \\
\hline 52. LP ( 2)Se 7 & $/ 192 . \mathrm{BD}^{*}(2) \mathrm{C} 2-\mathrm{C} 3$ & 1.46 & 0.23 & 0.018 \\
\hline 52. LP ( 2)Se 7 & /204. BD*( 1) C $8-\mathrm{H} 18$ & 2.50 & 0.70 & 0.038 \\
\hline 52. LP ( 2)Se 7 & /205. BD*( 1) C $8-\mathrm{H} 19$ & 2.79 & 0.68 & 0.040 \\
\hline 52. LP ( 2)Se 7 & /206. BD*( 1) C $9-\mathrm{O} 10$ & 0.55 & 0.82 & 0.019 \\
\hline 52. LP ( 2)Se 7 & /208. BD*( 1) C $9-\mathrm{H} 15$ & 2.57 & 0.65 & $0.037(*)$ \\
\hline 53. LP ( 1) O 10 & /139. RY*( 1) C 9 & 11.91 & 1.48 & 0.119 \\
\hline 53. LP ( 1) O 10 & /195. BD*( 1) C 3-C 9 & 1.59 & 1.12 & 0.038 \\
\hline 53. LP ( 1) O 10 & /208. BD*( 1) C $9-\mathrm{H} 15$ & 1.12 & 1.12 & 0.032 \\
\hline 54. LP( 2) O 10 & /140. RY*( 2) C 9 & 2.77 & 2.24 & 0.072 \\
\hline 54. LP ( 2) O 10 & /143. RY*( 5) C 9 & 1.00 & 1.66 & 0.037 \\
\hline 54. LP ( 2) O 10 & /181. RY*( 1) H 15 & 0.53 & 0.96 & 0.021 \\
\hline 54. LP ( 2) O 10 & /195. BD*( 1) C 3-C 9 & 20.13 & 0.69 & 0.106 \\
\hline 54. LP ( 2) O 10 & /198. BD*( 1) C 4 -H 13 & 0.54 & 0.74 & 0.018 \\
\hline 54. LP ( 2) O 10 & /208. BD*( 1) C 9-H 15 & 19.33 & 0.69 & 0.105 \\
\hline 55. LP ( 1) O 12 & /159. RY*( 1) C 11 & 11.89 & 1.48 & 0.118 \\
\hline 55. LP ( 1) O 12 & /190. BD*( 1) C $1-\mathrm{C} 11$ & 1.91 & 1.12 & 0.042 \\
\hline 55. LP ( 1) O 12 & /204. BD*( 1) C 8 - H 18 & 0.52 & 1.17 & 0.022 \\
\hline 55. LP ( 1) O 12 & /211. BD*( 1) C $11-\mathrm{H} 16$ & 1.01 & 1.10 & 0.030 \\
\hline 56. LP ( 2) O 12 & /160. RY*( 2) C 11 & 2.73 & 2.22 & 0.072 \\
\hline 56. LP ( 2) O 12 & /163. RY*( 5) C 11 & 1.24 & 1.63 & 0.041 \\
\hline 56. LP ( 2) O 12 & /182. RY*( 1) H 16 & 0.55 & 0.91 & 0.021 \\
\hline 56. LP ( 2) O 12 & /190. BD*( 1) C 1 -C 11 & 19.49 & 0.69 & 0.105 \\
\hline 56. LP ( 2) O 12 & /204. BD*( 1) C 8 - H 18 & 0.58 & 0.74 & 0.019 \\
\hline 56. LP ( 2) O 12 & /211. BD*( 1) C $11-$ H 16 & 21.18 & $\mathbf{0 . 6 7}$ & $\mathbf{0 . 1 0 8}$ \\
\hline
\end{tabular}




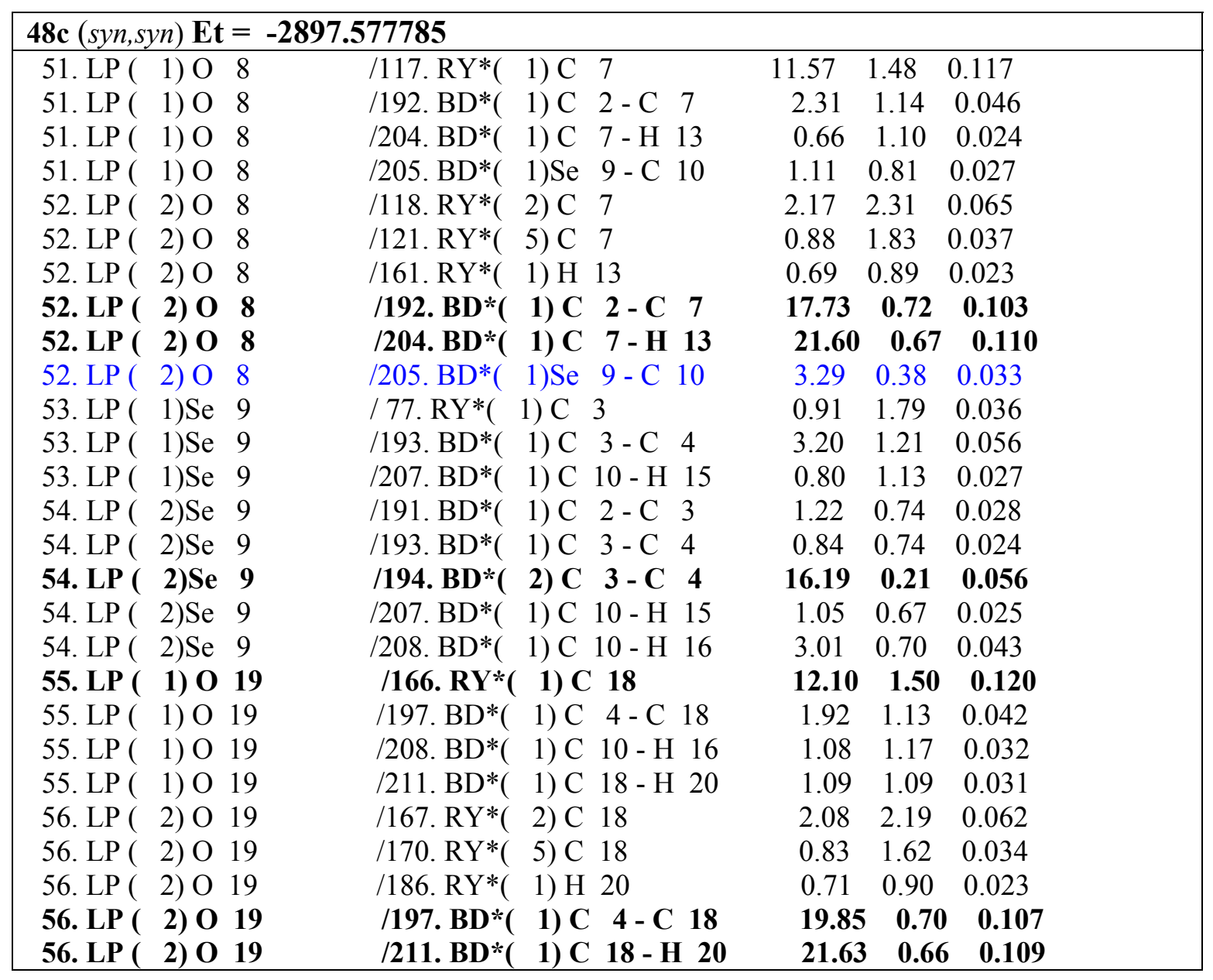




\begin{tabular}{|c|c|c|c|c|c|c|c|}
\hline \multicolumn{8}{|c|}{ 50a (syn, - SeMe in-plane) $\mathbf{E t}=\mathbf{- 2 7 8 4 . 2 6 7 0 1 5}$} \\
\hline 46. LP ( & 1)Se & 1 & / 72. RY*( & 1) $\mathrm{C} 3$ & 1.07 & 1.80 & 0.039 \\
\hline 46. LP ( & 1) $\mathrm{Se}$ & 1 & /164. BD*( & 1) $\mathrm{C} 3-\mathrm{C} \quad 4$ & 2.66 & 1.25 & 0.052 \\
\hline 46. LP ( & 1) $\mathrm{Se}$ & 1 & /179. BD*( & 1) $\mathrm{C} 9-\mathrm{H} 15$ & 0.73 & 1.14 & 0.026 \\
\hline 46. LP ( & 1)Se & 1 & $/ 180 . \mathrm{BD} *($ & 1) $\mathrm{C} 9-\mathrm{H} 16$ & 0.72 & 1.14 & 0.026 \\
\hline 47. LP ( & 2)Se & 1 & / 74. RY*( & 3) $\mathrm{C} 3$ & 0.61 & 1.36 & 0.027 \\
\hline 47. LP ( & 2)Se & 1 & /166. BD*( & 2) $\mathrm{C} \quad 3-\mathrm{C} \quad 8$ & 17.59 & 0.23 & 0.061 \\
\hline 47. LP ( & 2) $\mathrm{Se}$ & 1 & /179. BD*( & 1) $\mathrm{C} 9-\mathrm{H} 15$ & 2.60 & 0.66 & 0.039 \\
\hline 47. LP ( & 2) $\mathrm{Se}$ & 1 & $/ 180 . \mathrm{BD} *($ & 1) $\mathrm{C} 9-\mathrm{H} 16$ & 2.60 & 0.66 & 0.039 \\
\hline 48. LP ( & 1) 0 & 2 & /142. RY*( & 1) $\mathrm{C} 10$ & 11.27 & 1.48 & 0.116 \\
\hline 48. LP ( & 1) $\mathrm{O}$ & 2 & /161. BD*( & 1)Se 1 - C 9 & 1.75 & 0.80 & 0.034 \\
\hline 48. LP ( & 1) $\mathrm{O}$ & 2 & /177. BD*( & 1) $\mathrm{C} \quad 8$ - $\mathrm{C} \quad 10$ & 2.72 & 1.15 & 0.050 \\
\hline 49. LP ( & 2) $\mathrm{O}$ & 2 & /143. RY*( & 2) $\mathrm{C} 10$ & 2.17 & 2.36 & 0.066 \\
\hline 49. LP ( & 2) $\mathrm{O}$ & 2 & /146. RY*( & 5) C 10 & 0.75 & 1.83 & 0.034 \\
\hline 49. LP ( & 2) $\mathrm{O}$ & 2 & /154. RY*( & 1) $\mathrm{H} 13$ & 0.68 & 0.89 & 0.023 \\
\hline 49. LP ( & 2) $\mathrm{O}$ & 2 & /161. BD*( & 1)Se 1 - C 9 & 5.57 & 0.38 & 0.042 \\
\hline 49. LP ( & 2) $\mathrm{O}$ & 2 & /177. BD*( & 1) $\mathrm{C} 8$-C 10 & 15.96 & 0.74 & 0.099 \\
\hline 49. LP ( & 2) $\mathrm{O}$ & 2 & /181. BD*( & 1) $\mathrm{C} 10$ - H 13 & 21.23 & 0.68 & 0.109 \\
\hline
\end{tabular}

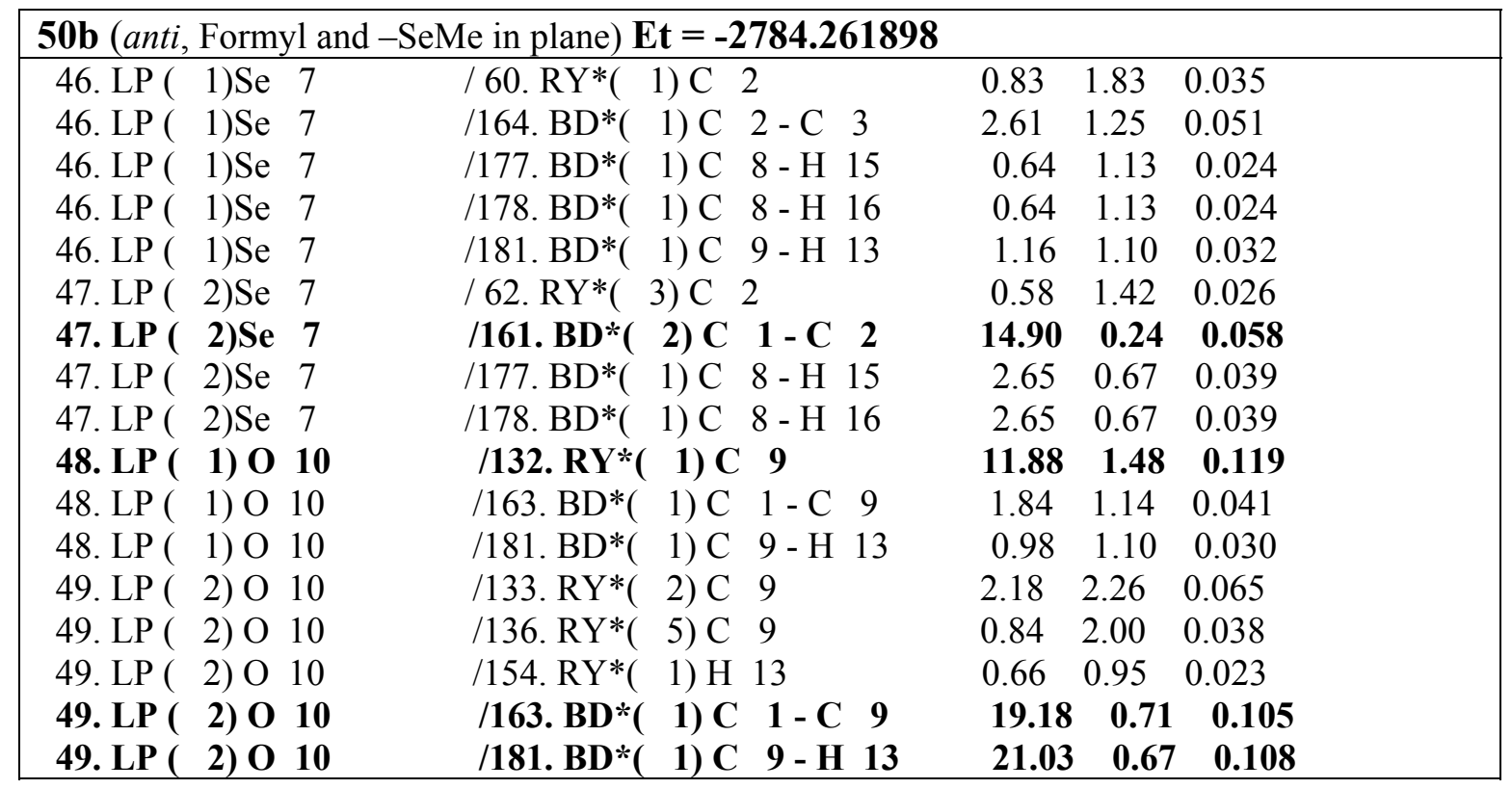




\begin{tabular}{|c|c|c|c|c|c|c|c|c|c|}
\hline \multicolumn{10}{|c|}{ 51a $($ syn, - SeSe in-plane with formyl) $\mathbf{E t}=\mathbf{- 5 1 8 3 . 6 7 8 6 3 3}$} \\
\hline 61. LP ( & 1) $\mathrm{O}$ & 8 & /127. RY*( & 1) $\mathrm{C}$ & 7 & & 10.85 & 1.49 & 0.114 \\
\hline 61. LP ( & 1) $\mathrm{O}$ & 8 & /197. BD*( & 1) $\mathrm{C}$ & $3-\mathrm{C}$ & 7 & 3.28 & 1.16 & 0.055 \\
\hline 61. LP ( & 1) $\mathrm{O}$ & 8 & /207. BD*( & 1) $\mathrm{Se}$ & 9 -Se 1 & 10 & 2.52 & 0.70 & 0.038 \\
\hline 62. LP ( & 2) $\mathrm{O}$ & 8 & /128. RY*( & 2) $\mathrm{C}$ & 7 & & 2.16 & 2.43 & 0.067 \\
\hline 62. LP ( & 2) $\mathrm{O}$ & 8 & /131. RY*( & 5) $\mathrm{C}$ & & & 0.57 & 1.80 & 0.030 \\
\hline 62. LP ( & 2) $\mathrm{O}$ & 8 & /182. RY*( & 1) $\mathrm{H}$ & 13 & & 0.63 & 0.90 & 0.022 \\
\hline 62. LP & 2) $\mathrm{O}$ & 8 & /197. BD*( & 1) $\mathrm{C}$ & $3-C$ & 7 & 13.77 & 0.76 & 0.094 \\
\hline 62. LP ( & 2) $\mathrm{O}$ & 8 & /199. BD*( & 1) $\mathrm{C}$ & $4-\mathrm{Se}$ & 9 & 0.51 & 0.40 & 0.013 \\
\hline 62. LP & 2) $\mathrm{O}$ & 8 & /206. BD*( & 1) $\mathrm{C}$ & $7-\mathbf{H}$ & 13 & 20.21 & 0.70 & 0.109 \\
\hline 62. LP & 2) $\mathrm{O}$ & 8 & /207. BD*( & 1) $\mathrm{Sc}$ & 9 -Se & 10 & 10.06 & 0.30 & 0.050 \\
\hline 63. LP ( & 1) $\mathrm{Se}$ & 9 & / 97. RY*( & 1) $\mathrm{C}$ & & & 0.88 & 1.79 & 0.035 \\
\hline 63. LP ( & 1) $\mathrm{Se}$ & 9 & /198. BD*( & 1) $\mathrm{C}$ & $4-C$ & & 2.21 & 1.30 & 0.048 \\
\hline 64. LP ( & 2)Se & 9 & /159. RY*( & 1) $\mathrm{Se}$ & 10 & & 1.22 & 0.79 & 0.029 \\
\hline 64. LP ( & 2) $\mathrm{Se}$ & 9 & /196. BD*( & 2) $\mathrm{C}$ & $3-C$ & 4 & 14.77 & 0.24 & 0.058 \\
\hline 64. LP ( & 2) $\mathrm{Se}$ & 9 & /208. BD*( & 1) $\mathrm{Se}$ & $10-\mathrm{C}$ & 11 & 3.92 & 0.33 & 0.033 \\
\hline 64. LP ( & 2) $\mathrm{Se}$ & 9 & /209. BD*( & 1) $\mathrm{C}$ & $11-\mathrm{H}$ & 17 & 0.58 & 0.68 & 0.018 \\
\hline 65. LP ( & 1) $\mathrm{Se}$ & 10 & /202. BD* & 1) $\mathrm{C}$ & $5-\mathrm{H}$ & 12 & 0.62 & 1.17 & 0.024 \\
\hline 66. LP ( & 2) $\mathrm{Se}$ & 10 & /147. RY* & 1) $\mathrm{S}$ & 9 & & 0.52 & 0.97 & 0.020 \\
\hline 66. LP ( & 2)Se & 10 & /199. BD* & 1) ( & $4-\mathrm{Se}$ & & 5.30 & 0.34 & 0.038 \\
\hline 66. LP ( & 2) $\mathrm{Se}$ & 10 & /202. BD* & 1) $\mathrm{C}$ & $5-\mathrm{H}$ & 12 & 3.51 & 0.70 & 0.045 \\
\hline 66. LP ( & 2) $\mathrm{Se}$ & 10 & $/ 210 . \mathrm{BD}^{*}$ & 1) $C$ & $11-\mathrm{H}$ & 18 & 2.59 & 0.68 & 0.038 \\
\hline 66. LP ( & 2) $\mathrm{Se}$ & 10 & $/ 211 . \mathrm{BD} *($ & 1) $\mathrm{C}$ & $11-\mathrm{H}$ & 19 & 2.23 & 0.69 & 0.035 \\
\hline
\end{tabular}

\begin{tabular}{|c|c|c|c|c|c|c|c|c|}
\hline \multicolumn{9}{|c|}{ 51b (anti, - SeSe in-plane with formyl) $\mathbf{E t}=\mathbf{- 5 1 8 3 . 6 7 1 0 3 1}$} \\
\hline 61. LP ( & 1) $\mathrm{C}$ & 8 & /127. RY*( & 1) $\mathrm{C}$ & 7 & 11.93 & 1.48 & 0.119 \\
\hline 61. LP ( & 1) $\mathrm{C}$ & 8 & /200. BD*( & 1) $\mathrm{C}$ & $4-\mathrm{C} 7$ & 1.89 & 1.14 & 0.042 \\
\hline 61. LP ( & 1) $\mathrm{C}$ & 8 & /206. BD*( & 1) $\mathrm{C}$ & $7-\mathrm{H} 13$ & 0.98 & 1.10 & 0.030 \\
\hline 62. LP ( & 2) $\mathrm{C}$ & 8 & /128. RY*( & 2) $\mathrm{C}$ & 7 & 2.17 & 2.25 & 0.064 \\
\hline 62. LP & 2) $\mathrm{C}$ & 8 & $/ 131 . \mathrm{RY} *($ & 5) $\mathrm{C}$ & 7 & 0.84 & 2.03 & 0.038 \\
\hline 62. LP & 2) $\mathrm{C}$ & 8 & /182. RY*( & 1) $\mathrm{H}$ & 13 & 0.66 & 0.95 & 0.023 \\
\hline 62. LP ( & 2) ( & 8 & /200. BD*( & 1) $\mathrm{C}$ & 4-C 7 & 19.10 & 0.71 & 0.105 \\
\hline 62. LP ( & 2) ( & 8 & /206. BD*( & 1) $\mathrm{C}$ & $7-$ H 13 & 21.37 & 0.67 & 0.108 \\
\hline 63. LP ( & 1) $\mathrm{S}$ & 9 & /107. RY*( & 1) $\mathrm{C}$ & 5 & 0.61 & 1.83 & 0.030 \\
\hline 63. LP ( & 1) $\mathrm{S}$ & 9 & $/ 201 . \mathrm{BD} *($ & 1) $\mathrm{C}$ & $5-C 6$ & 2.15 & 1.29 & 0.047 \\
\hline 63. LP ( & 1) $\mathrm{S}$ & 9 & /206. BD*( & 1) $\mathrm{C}$ & $7-\mathrm{H} 13$ & 1.20 & 1.13 & $0.033(*)$ \\
\hline 64. LP ( & 2) $\mathrm{S}$ & 9 & /159. RY*( & 1) $\mathrm{Se}$ & & 1.42 & 0.79 & 0.031 \\
\hline 64. LP ( & 2)S & 9 & /199. BD*( & 2) $\mathrm{C}$ & 4 - C 5 & 12.32 & 0.26 & 0.055 \\
\hline 64. LP ( & 2) $\mathrm{S}$ & 9 & /208. BD*( & 1)Se & $10-\mathrm{C} 11$ & 4.54 & 0.32 & 0.035 \\
\hline 64. LP ( & 2) $\mathrm{S}$ & 9 & /209. BD*( & 1) $\mathrm{C}$ & $11-\mathrm{H} 17$ & 0.61 & 0.68 & 0.019 \\
\hline 65. LP & 1) $\mathrm{S}$ & 10 & /203. BD* & 1) $\mathrm{C}$ & $6-\mathrm{H} 12$ & 0.58 & 1.19 & 0.024 \\
\hline 66. LP ( & 2)S & 10 & /147. RY* & 1) $\mathrm{Se}$ & 9 & 1.02 & 0.96 & 0.029 \\
\hline 66. LP ( & 2)S & 10 & /202. BD* & 1) $\mathrm{C}$ & 5 -Se 9 & 6.03 & 0.34 & 0.040 \\
\hline 66. LP ( & 2) $\mathrm{S}$ & 10 & /203. BD* & 1) $\mathrm{C}$ & $6-\mathrm{H} 12$ & 3.13 & 0.71 & 0.043 \\
\hline 66. LP ( & 2) $\mathrm{S}$ & 10 & $/ 210 . \mathrm{BD}^{*}$ & 1) $\mathrm{C}$ & $11-\mathrm{H} 18$ & 2.44 & 0.69 & 0.037 \\
\hline 66. LP ( & 2) $\mathrm{S}$ & 10 & /211. BD* & 1) $\mathrm{C}$ & $11-\mathrm{H} 19$ & 2.26 & 0.69 & 0.036 \\
\hline
\end{tabular}




\section{Tables for crystallographic data of 25}

Table 1. Crystal data and structure refinement for $\mathbf{2 5}$.

Identification code

Empirical formula

Formula weight

Temperature

Wavelength

Crystal system

Space group

Unit cell dimensions

Volume

$\mathrm{Z}$

Density (calculated)

Absorption coefficient

$\mathrm{F}(000)$

Crystal size

Theta range for data collection

Index ranges

Reflections collected

Independent reflections

Completeness to theta $=28.34^{\circ}$

Absorption correction

Max. and min. transmission

Refinement method

Data / restraints / parameters

Goodness-of-fit on $\mathrm{F}^{2}$

Final $R$ indices $[\mathrm{I}>2 \operatorname{sigma}(\mathrm{I})]$

$\mathrm{R}$ indices (all data)

Absolute structure parameter

Largest diff. peak and hole procho $2 \mathrm{~m}$

C32 H42 O8 Se2

712.58

293(2) K

$0.71073 \AA$

Orthorhombic

P2(1)2(1)2(1)

$$
\begin{array}{ll}
\mathrm{a}=10.052(4) \AA & \mathrm{a}=90^{\circ} . \\
\mathrm{b}=13.982(6) \AA & \mathrm{b}=90^{\circ} . \\
\mathrm{c}=22.653(10) \AA & \mathrm{g}=90^{\circ} .
\end{array}
$$

\section{4(2) $\AA^{3}$}

4

$1.487 \mathrm{Mg} / \mathrm{m}^{3}$

$2.371 \mathrm{~mm}^{-1}$

1464

$0.45 \times 0.5 \times 0.55 \mathrm{~mm}^{3}$

1.71 to $28.34^{\circ}$.

$-13<=\mathrm{h}<=13,-18<=\mathrm{k}<=17,-29<=\mathrm{l}<=30$

22850

$7731[\mathrm{R}($ int $)=0.1159]$

$98.0 \%$

Sadabs

1.000000 and 0.3797

Full-matrix least-squares on $\mathrm{F}^{2}$

7731 / 0 / 385

0.852

$\mathrm{R} 1=0.0566, \mathrm{wR} 2=0.1169$

$\mathrm{R} 1=0.1400, \mathrm{wR} 2=0.1382$

$-0.001(15)$

1.383 and -1.053 e. $\AA^{-3}$ 
Table 2. Atomic coordinates ( x 10 $)$ and equivalent isotropic displacement parameters $\left(\AA^{2} \times 10^{3}\right)$ for 25. $U(e q)$ is defined as one third of the trace of the orthogonalized Uij tensor.

\begin{tabular}{|c|c|c|c|c|}
\hline & $\mathrm{x}$ & $\mathrm{y}$ & $\mathrm{z}$ & $\mathrm{U}(\mathrm{eq})$ \\
\hline $\operatorname{Se}(1 \mathrm{~A})$ & 10638(1) & 10832(1) & $3905(1)$ & $44(1)$ \\
\hline $\mathrm{Se}(1 \mathrm{~B})$ & $9137(1)$ & $10715(1)$ & $3139(1)$ & $45(1)$ \\
\hline $\mathrm{O}(1 \mathrm{~A})$ & $9817(6)$ & $8177(3)$ & $4911(2)$ & $70(2)$ \\
\hline $\mathrm{O}(2 \mathrm{~A})$ & $11623(5)$ & $9072(3)$ & $4856(2)$ & $61(1)$ \\
\hline $\mathrm{O}(3 \mathrm{~A})$ & $7241(6)$ & $12342(3)$ & $4173(2)$ & $72(2)$ \\
\hline $\mathrm{O}(4 \mathrm{~A})$ & $8565(6)$ & $13134(3)$ & $4796(2)$ & $66(2)$ \\
\hline $\mathrm{O}(1 \mathrm{~B})$ & $6134(7)$ & $9584(4)$ & $4275(2)$ & $85(2)$ \\
\hline $\mathrm{O}(2 \mathrm{~B})$ & $5398(6)$ & $10070(5)$ & $3426(2)$ & $96(2)$ \\
\hline $\mathrm{O}(3 \mathrm{~B})$ & $11170(6)$ & $8242(4)$ & $2307(2)$ & $64(2)$ \\
\hline $\mathrm{O}(4 \mathrm{~B})$ & $11686(6)$ & $8662(6)$ & $3227(3)$ & $96(2)$ \\
\hline $\mathrm{C}(1 \mathrm{~A})$ & $9478(6)$ & $10709(5)$ & $4562(2)$ & $37(1)$ \\
\hline $\mathrm{C}(2 \mathrm{~A})$ & $9541(6)$ & $9855(4)$ & $4895(3)$ & $36(2)$ \\
\hline $\mathrm{C}(3 \mathrm{~A})$ & $8849(6)$ & $9793(5)$ & $5408(3)$ & $44(2)$ \\
\hline $\mathrm{C}(4 \mathrm{~A})$ & $8101(6)$ & $10531(5)$ & $5639(3)$ & $42(2)$ \\
\hline $\mathrm{C}(5 \mathrm{~A})$ & $8016(7)$ & $11352(5)$ & $5293(3)$ & $42(2)$ \\
\hline $\mathrm{C}(6 \mathrm{~A})$ & $8718(6)$ & $11451(4)$ & $4775(3)$ & $38(2)$ \\
\hline $\mathrm{C}(7 \mathrm{~A})$ & $10322(6)$ & $9019(4)$ & $4676(3)$ & $42(2)$ \\
\hline $\mathrm{C}(8 \mathrm{~A})$ & 10859(10) & $7515(6)$ & $4842(4)$ & $90(3)$ \\
\hline $\mathrm{C}(9 \mathrm{~A})$ & $12112(10)$ & $8111(7)$ & $4827(4)$ & $92(3)$ \\
\hline $\mathrm{C}(10 \mathrm{~A})$ & $8505(8)$ & $12342(5)$ & $4430(3)$ & $49(2)$ \\
\hline $\mathrm{C}(11 \mathrm{~A})$ & $6695(11)$ & $13241(8)$ & $4272(5)$ & 111(4) \\
\hline $\mathrm{C}(12 \mathrm{~A})$ & 7634(10) & $13785(6)$ & $4595(5)$ & $88(3)$ \\
\hline $\mathrm{C}(13 \mathrm{~A})$ & $7395(7)$ & $10510(5)$ & $6221(3)$ & $54(2)$ \\
\hline $\mathrm{C}(14 \mathrm{~A})$ & 7304(9) & $9488(6)$ & $6467(4)$ & $91(3)$ \\
\hline $\mathrm{C}(15 \mathrm{~A})$ & $8190(12)$ & $11103(9)$ & $6660(4)$ & $133(5)$ \\
\hline $\mathrm{C}(16 \mathrm{~A})$ & $6050(9)$ & $10912(9)$ & $6189(4)$ & $126(4)$ \\
\hline$C(1 B)$ & $8636(6)$ & $9394(4)$ & $3208(3)$ & $40(2)$ \\
\hline $\mathrm{C}(2 \mathrm{~B})$ & $7418(6)$ & $9187(5)$ & $3440(3)$ & $42(2)$ \\
\hline$C(3 B)$ & $6955(7)$ & $8250(5)$ & $3419(3)$ & $51(2)$ \\
\hline$C(4 B)$ & $7681(7)$ & $7528(4)$ & $3179(3)$ & $43(2)$ \\
\hline $\mathrm{C}(5 \mathrm{~B})$ & $8938(7)$ & $7741(5)$ & $2973(3)$ & $48(2)$ \\
\hline
\end{tabular}




$\begin{array}{lccrr}\mathrm{C}(6 \mathrm{~B}) & 9433(7) & 8674(4) & 2978(3) & 37(2) \\ \mathrm{C}(7 \mathrm{~B}) & 6540(7) & 9908(5) & 3736(3) & 46(2) \\ \mathrm{C}(8 \mathrm{~B}) & 4988(10) & 10117(7) & 4402(5) & 96(4) \\ \mathrm{C}(9 \mathrm{~B}) & 4491(9) & 10425(9) & 3847(5) & 113(4) \\ \mathrm{C}(10 \mathrm{~B}) & 10802(8) & 8834(5) & 2766(3) & 58(2) \\ \mathrm{C}(11 \mathrm{~B}) & 12515(10) & 8033(8) & 2393(6) & 106(4) \\ \mathrm{C}(12 \mathrm{~B}) & 12829(10) & 8247(8) & 2971(5) & 96(3) \\ \mathrm{C}(13 \mathrm{~B}) & 7185(8) & 6514(5) & 3142(4) & 61(2) \\ \mathrm{C}(14 \mathrm{~B}) & 5991(11) & 6344(7) & 3483(5) & 137(5) \\ \mathrm{C}(15 \mathrm{~B}) & 7039(11) & 6268(7) & 2501(4) & 108(4) \\ \mathrm{C}(16 \mathrm{~B}) & 8237(10) & 5836(6) & 3391(5) & 105(3)\end{array}$


Table 3. Bond lengths $[\AA]$ and angles $\left[{ }^{\circ}\right]$ for $\mathbf{2 5}$.

\begin{tabular}{ll}
\hline $\mathrm{Se}(1 \mathrm{~A})-\mathrm{C}(1 \mathrm{~A})$ & $1.899(6)$ \\
$\mathrm{Se}(1 \mathrm{~A})-\mathrm{Se}(1 \mathrm{~B})$ & $2.3052(12)$ \\
$\mathrm{Se}(1 \mathrm{~B})-\mathrm{C}(1 \mathrm{~B})$ & $1.920(6)$ \\
$\mathrm{O}(1 \mathrm{~A})-\mathrm{C}(7 \mathrm{~A})$ & $1.388(7)$ \\
$\mathrm{O}(1 \mathrm{~A})-\mathrm{C}(8 \mathrm{~A})$ & $1.405(9)$ \\
$\mathrm{O}(2 \mathrm{~A})-\mathrm{C}(7 \mathrm{~A})$ & $1.371(7)$ \\
$\mathrm{O}(2 \mathrm{~A})-\mathrm{C}(9 \mathrm{~A})$ & $1.433(9)$ \\
$\mathrm{O}(3 \mathrm{~A})-\mathrm{C}(11 \mathrm{~A})$ & $1.389(10)$ \\
$\mathrm{O}(3 \mathrm{~A})-\mathrm{C}(10 \mathrm{~A})$ & $1.398(9)$ \\
$\mathrm{O}(4 \mathrm{~A})-\mathrm{C}(12 \mathrm{~A})$ & $1.382(10)$ \\
$\mathrm{O}(4 \mathrm{~A})-\mathrm{C}(10 \mathrm{~A})$ & $1.385(8)$ \\
$\mathrm{O}(1 \mathrm{~B})-\mathrm{C}(7 \mathrm{~B})$ & $1.364(8)$ \\
$\mathrm{O}(1 \mathrm{~B})-\mathrm{C}(8 \mathrm{~B})$ & $1.403(10)$ \\
$\mathrm{O}(2 \mathrm{~B})-\mathrm{C}(7 \mathrm{~B})$ & $1.366(8)$ \\
$\mathrm{O}(2 \mathrm{~B})-\mathrm{C}(9 \mathrm{~B})$ & $1.411(10)$ \\
$\mathrm{O}(3 \mathrm{~B})-\mathrm{C}(10 \mathrm{~B})$ & $1.380(8)$ \\
$\mathrm{O}(3 \mathrm{~B})-\mathrm{C}(11 \mathrm{~B})$ & $1.396(11)$ \\
$\mathrm{O}(4 \mathrm{~B})-\mathrm{C}(10 \mathrm{~B})$ & $1.393(9)$ \\
$\mathrm{O}(4 \mathrm{~B})-\mathrm{C}(12 \mathrm{~B})$ & $1.412(11)$ \\
$\mathrm{C}(1 \mathrm{~A})-\mathrm{C}(6 \mathrm{~A})$ & $1.376(8)$ \\
$\mathrm{C}(1 \mathrm{~A})-\mathrm{C}(2 \mathrm{~A})$ & $1.414(8)$ \\
$\mathrm{C}(2 \mathrm{~A})-\mathrm{C}(3 \mathrm{~A})$ & $1.356(8)$ \\
$\mathrm{C}(2 \mathrm{~A})-\mathrm{C}(7 \mathrm{~A})$ & $1.493(8)$ \\
$\mathrm{C}(3 \mathrm{~A})-\mathrm{C}(4 \mathrm{~A})$ & $1.380(8)$ \\
$\mathrm{C}(4 \mathrm{~A})-\mathrm{C}(5 \mathrm{~A})$ & $1.392(9)$ \\
$\mathrm{C}(4 \mathrm{~A})-\mathrm{C}(13 \mathrm{~A})$ & $1.498(9)$ \\
$\mathrm{C}(5 \mathrm{~A})-\mathrm{C}(6 \mathrm{~A})$ & $1.375(9)$ \\
$\mathrm{C}(6 \mathrm{~A})-\mathrm{C}(10 \mathrm{~A})$ & $1.487(9)$ \\
$\mathrm{C}(8 \mathrm{~A})-\mathrm{C}(9 \mathrm{~A})$ & $1.510(12)$ \\
$\mathrm{C}(11 \mathrm{~A})-\mathrm{C}(12 \mathrm{~A})$ & $1.416(13)$ \\
$\mathrm{C}(13 \mathrm{~A})-\mathrm{C}(16 \mathrm{~A})$ & $1.466(10)$ \\
$\mathrm{C}(13 \mathrm{~A})-\mathrm{C}(15 \mathrm{~A})$ & $1.523(11)$ \\
$\mathrm{C}(13 \mathrm{~A})-\mathrm{C}(14 \mathrm{~A})$ & $1.536(10)$ \\
$\mathrm{C}(1 \mathrm{~B})-\mathrm{C}(2 \mathrm{~B})$ & $1.363(8)$ \\
&
\end{tabular}




\begin{tabular}{|c|c|}
\hline$C(1 B)-C(6 B)$ & $1.389(9)$ \\
\hline$C(2 B)-C(3 B)$ & $1.391(9)$ \\
\hline$C(2 B)-C(7 B)$ & $1.499(8)$ \\
\hline $\mathrm{C}(3 \mathrm{~B})-\mathrm{C}(4 \mathrm{~B})$ & $1.360(9)$ \\
\hline $\mathrm{C}(4 \mathrm{~B})-\mathrm{C}(5 \mathrm{~B})$ & $1.379(9)$ \\
\hline $\mathrm{C}(4 \mathrm{~B})-\mathrm{C}(13 \mathrm{~B})$ & $1.506(9)$ \\
\hline $\mathrm{C}(5 \mathrm{~B})-\mathrm{C}(6 \mathrm{~B})$ & $1.396(9)$ \\
\hline $\mathrm{C}(6 \mathrm{~B})-\mathrm{C}(10 \mathrm{~B})$ & $1.474(9)$ \\
\hline $\mathrm{C}(8 \mathrm{~B})-\mathrm{C}(9 \mathrm{~B})$ & $1.420(12)$ \\
\hline $\mathrm{C}(11 \mathrm{~B})-\mathrm{C}(12 \mathrm{~B})$ & $1.380(12)$ \\
\hline$C(13 B)-C(14 B)$ & $1.447(11)$ \\
\hline $\mathrm{C}(13 \mathrm{~B})-\mathrm{C}(15 \mathrm{~B})$ & $1.500(11)$ \\
\hline$C(13 B)-C(16 B)$ & $1.527(11)$ \\
\hline $\mathrm{C}(1 \mathrm{~A})-\mathrm{Se}(1 \mathrm{~A})-\mathrm{Se}(1 \mathrm{~B})$ & $100.45(17)$ \\
\hline $\mathrm{C}(1 \mathrm{~B})-\mathrm{Se}(1 \mathrm{~B})-\mathrm{Se}(1 \mathrm{~A})$ & $100.33(19)$ \\
\hline $\mathrm{C}(7 \mathrm{~A})-\mathrm{O}(1 \mathrm{~A})-\mathrm{C}(8 \mathrm{~A})$ & 104.1(6) \\
\hline $\mathrm{C}(7 \mathrm{~A})-\mathrm{O}(2 \mathrm{~A})-\mathrm{C}(9 \mathrm{~A})$ & $105.3(6)$ \\
\hline$C(11 A)-O(3 A)-C(10 A)$ & $107.0(7)$ \\
\hline$C(12 A)-O(4 A)-C(10 A)$ & $107.4(6)$ \\
\hline $\mathrm{C}(7 \mathrm{~B})-\mathrm{O}(1 \mathrm{~B})-\mathrm{C}(8 \mathrm{~B})$ & $104.7(6)$ \\
\hline $\mathrm{C}(7 \mathrm{~B})-\mathrm{O}(2 \mathrm{~B})-\mathrm{C}(9 \mathrm{~B})$ & $104.7(6)$ \\
\hline $\mathrm{C}(10 \mathrm{~B})-\mathrm{O}(3 \mathrm{~B})-\mathrm{C}(11 \mathrm{~B})$ & $106.4(7)$ \\
\hline $\mathrm{C}(10 \mathrm{~B})-\mathrm{O}(4 \mathrm{~B})-\mathrm{C}(12 \mathrm{~B})$ & $106.4(7)$ \\
\hline$C(6 A)-C(1 A)-C(2 A)$ & $118.3(5)$ \\
\hline$C(6 \mathrm{~A})-\mathrm{C}(1 \mathrm{~A})-\mathrm{Se}(1 \mathrm{~A})$ & $123.2(5)$ \\
\hline$C(2 A)-C(1 A)-S e(1 A)$ & $117.9(5)$ \\
\hline$C(3 A)-C(2 A)-C(1 A)$ & $119.2(6)$ \\
\hline$C(3 A)-C(2 A)-C(7 A)$ & $120.3(6)$ \\
\hline$C(1 \mathrm{~A})-\mathrm{C}(2 \mathrm{~A})-\mathrm{C}(7 \mathrm{~A})$ & $120.5(6)$ \\
\hline$C(2 A)-C(3 A)-C(4 A)$ & $123.8(6)$ \\
\hline$C(3 A)-C(4 A)-C(5 A)$ & $115.9(6)$ \\
\hline$C(3 A)-C(4 A)-C(13 A)$ & $125.2(6)$ \\
\hline$C(5 A)-C(4 A)-C(13 A)$ & $118.9(6)$ \\
\hline$C(6 A)-C(5 A)-C(4 A)$ & $122.0(6)$ \\
\hline$C(5 A)-C(6 A)-C(1 A)$ & $120.6(6)$ \\
\hline
\end{tabular}




$\begin{array}{ll}\mathrm{C}(5 \mathrm{~A})-\mathrm{C}(6 \mathrm{~A})-\mathrm{C}(10 \mathrm{~A}) & 117.3(6) \\ \mathrm{C}(1 \mathrm{~A})-\mathrm{C}(6 \mathrm{~A})-\mathrm{C}(10 \mathrm{~A}) & 121.8(6) \\ \mathrm{O}(2 \mathrm{~A})-\mathrm{C}(7 \mathrm{~A})-\mathrm{O}(1 \mathrm{~A}) & 106.3(6) \\ \mathrm{O}(2 \mathrm{~A})-\mathrm{C}(7 \mathrm{~A})-\mathrm{C}(2 \mathrm{~A}) & 111.2(5) \\ \mathrm{O}(1 \mathrm{~A})-\mathrm{C}(7 \mathrm{~A})-\mathrm{C}(2 \mathrm{~A}) & 110.2(5) \\ \mathrm{O}(1 \mathrm{~A})-\mathrm{C}(8 \mathrm{~A})-\mathrm{C}(9 \mathrm{~A}) & 105.2(6) \\ \mathrm{O}(2 \mathrm{~A})-\mathrm{C}(9 \mathrm{~A})-\mathrm{C}(8 \mathrm{~A}) & 103.3(7) \\ \mathrm{O}(4 \mathrm{~A})-\mathrm{C}(10 \mathrm{~A})-\mathrm{O}(3 \mathrm{~A}) & 106.8(6) \\ \mathrm{O}(4 \mathrm{~A})-\mathrm{C}(10 \mathrm{~A})-\mathrm{C}(6 \mathrm{~A}) & 110.5(6) \\ \mathrm{O}(3 \mathrm{~A})-\mathrm{C}(10 \mathrm{~A})-\mathrm{C}(6 \mathrm{~A}) & 110.5(6) \\ \mathrm{O}(3 \mathrm{~A})-\mathrm{C}(11 \mathrm{~A})-\mathrm{C}(12 \mathrm{~A}) & 107.8(8) \\ \mathrm{O}(4 \mathrm{~A})-\mathrm{C}(12 \mathrm{~A})-\mathrm{C}(11 \mathrm{~A}) & 105.6(7) \\ \mathrm{C}(16 \mathrm{~A})-\mathrm{C}(13 \mathrm{~A})-\mathrm{C}(4 \mathrm{~A}) & 112.6(6) \\ \mathrm{C}(16 \mathrm{~A})-\mathrm{C}(13 \mathrm{~A})-\mathrm{C}(15 \mathrm{~A}) & 107.9(8) \\ \mathrm{C}(4 \mathrm{~A})-\mathrm{C}(13 \mathrm{~A})-\mathrm{C}(15 \mathrm{~A}) & 108.4(7) \\ \mathrm{C}(16 \mathrm{~A})-\mathrm{C}(13 \mathrm{~A})-\mathrm{C}(14 \mathrm{~A}) & 108.7(7) \\ \mathrm{C}(4 \mathrm{~A})-\mathrm{C}(13 \mathrm{~A})-\mathrm{C}(14 \mathrm{~A}) & 111.5(6) \\ \mathrm{C}(15 \mathrm{~A})-\mathrm{C}(13 \mathrm{~A})-\mathrm{C}(14 \mathrm{~A}) & 107.6(8) \\ \mathrm{C}(2 \mathrm{~B})-\mathrm{C}(1 \mathrm{~B})-\mathrm{C}(6 \mathrm{~B}) & 120.6(6) \\ \mathrm{C}(2 \mathrm{~B})-\mathrm{C}(1 \mathrm{~B})-\mathrm{Se}(1 \mathrm{~B}) & 118.1(5) \\ \mathrm{C}(6 \mathrm{~B})-\mathrm{C}(1 \mathrm{~B})-\mathrm{Se}(1 \mathrm{~B}) & 121.0(4) \\ \mathrm{C}(1 \mathrm{~B})-\mathrm{C}(2 \mathrm{~B})-\mathrm{C}(3 \mathrm{~B}) & 119.2(6) \\ \mathrm{C}(1 \mathrm{~B})-\mathrm{C}(2 \mathrm{~B})-\mathrm{C}(7 \mathrm{~B}) & 123.9(6) \\ \mathrm{C}(3 \mathrm{~B})-\mathrm{C}(2 \mathrm{~B})-\mathrm{C}(7 \mathrm{~B}) & 116.8(6) \\ \mathrm{C}(4 \mathrm{~B})-\mathrm{C}(3 \mathrm{~B})-\mathrm{C}(2 \mathrm{~B}) & 122.2(6) \\ \mathrm{C}(3 \mathrm{~B})-\mathrm{C}(4 \mathrm{~B})-\mathrm{C}(5 \mathrm{~B}) & 117.8(6) \\ \mathrm{C}(3 \mathrm{~B})-\mathrm{C}(4 \mathrm{~B})-\mathrm{C}(13 \mathrm{~B}) & 122.9(6) \\ \mathrm{C}(5 \mathrm{~B})-\mathrm{C}(4 \mathrm{~B})-\mathrm{C}(13 \mathrm{~B}) & 119.2(6) \\ \mathrm{C}(4 \mathrm{~B})-\mathrm{C}(5 \mathrm{~B})-\mathrm{C}(6 \mathrm{~B}) & 121.7(6) \\ \mathrm{C}(1 \mathrm{~B})-\mathrm{C}(6 \mathrm{~B})-\mathrm{C}(5 \mathrm{~B}) & 118.3(6) \\ \mathrm{C}(1 \mathrm{~B})-\mathrm{C}(6 \mathrm{~B})-\mathrm{C}(10 \mathrm{~B}) & 123.4(6) \\ \mathrm{C}(5 \mathrm{~B})-\mathrm{C}(6 \mathrm{~B})-\mathrm{C}(10 \mathrm{~B}) & 118.2(6) \\ \mathrm{O}(1 \mathrm{~B})-\mathrm{C}(7 \mathrm{~B})-\mathrm{O}(2 \mathrm{~B}) & 105.3(6) \\ \mathrm{O}(1 \mathrm{~B})-\mathrm{C}(7 \mathrm{~B})-\mathrm{C}(2 \mathrm{~B}) & 110.7(6) \\ \mathrm{O}(2 \mathrm{~B})-\mathrm{C}(7 \mathrm{~B})-\mathrm{C}(2 \mathrm{~B}) & 112.1(6) \\ \mathrm{O}(1 \mathrm{~B})-\mathrm{C}(8 \mathrm{~B})-\mathrm{C}(9 \mathrm{~B}) & 105.5(7)\end{array}$




$\begin{array}{ll}\mathrm{O}(2 \mathrm{~B})-\mathrm{C}(9 \mathrm{~B})-\mathrm{C}(8 \mathrm{~B}) & 105.4(7) \\ \mathrm{O}(3 \mathrm{~B})-\mathrm{C}(10 \mathrm{~B})-\mathrm{O}(4 \mathrm{~B}) & 106.8(6) \\ \mathrm{O}(3 \mathrm{~B})-\mathrm{C}(10 \mathrm{~B})-\mathrm{C}(6 \mathrm{~B}) & 113.9(6) \\ \mathrm{O}(4 \mathrm{~B})-\mathrm{C}(10 \mathrm{~B})-\mathrm{C}(6 \mathrm{~B}) & 109.0(6) \\ \mathrm{C}(12 \mathrm{~B})-\mathrm{C}(11 \mathrm{~B})-\mathrm{O}(3 \mathrm{~B}) & 107.9(8) \\ \mathrm{C}(11 \mathrm{~B})-\mathrm{C}(12 \mathrm{~B})-\mathrm{O}(4 \mathrm{~B}) & 107.1(8) \\ \mathrm{C}(14 \mathrm{~B})-\mathrm{C}(13 \mathrm{~B})-\mathrm{C}(15 \mathrm{~B}) & 113.4(9) \\ \mathrm{C}(14 \mathrm{~B})-\mathrm{C}(13 \mathrm{~B})-\mathrm{C}(4 \mathrm{~B}) & 113.6(6) \\ \mathrm{C}(15 \mathrm{~B})-\mathrm{C}(13 \mathrm{~B})-\mathrm{C}(4 \mathrm{~B}) & 107.5(7) \\ \mathrm{C}(14 \mathrm{~B})-\mathrm{C}(13 \mathrm{~B})-\mathrm{C}(16 \mathrm{~B}) & 106.1(8) \\ \mathrm{C}(15 \mathrm{~B})-\mathrm{C}(13 \mathrm{~B})-\mathrm{C}(16 \mathrm{~B}) & 106.4(7) \\ \mathrm{C}(4 \mathrm{~B})-\mathrm{C}(13 \mathrm{~B})-\mathrm{C}(16 \mathrm{~B}) & 109.6(7)\end{array}$

Symmetry transformations used to generate equivalent atoms: 
Table 4. Anisotropic displacement parameters $\left(\AA^{2} \times 10^{3}\right)$ for 25. The anisotropic displacement factor exponent takes the form: $-2 p^{2}\left[h^{2} a^{* 2} U^{11}+\ldots+2 h k^{*} b^{*} U^{12}\right]$

\begin{tabular}{lcccccc}
\hline & $\mathrm{U} 11$ & $\mathrm{U}^{22}$ & $\mathrm{U}^{33}$ & $\mathrm{U}^{23}$ & $\mathrm{U} 13$ & $\mathrm{U} 12$ \\
\hline $\mathrm{Se}(1 \mathrm{~A})$ & $43(1)$ & $44(1)$ & $45(1)$ & $-3(1)$ & $9(1)$ & $-2(1)$ \\
$\mathrm{Se}(1 \mathrm{~B})$ & $61(1)$ & $33(1)$ & $43(1)$ & $4(1)$ & $1(1)$ & $1(1)$ \\
$\mathrm{O}(1 \mathrm{~A})$ & $88(4)$ & $24(3)$ & $99(4)$ & $-2(3)$ & $35(3)$ & $-3(3)$ \\
$\mathrm{O}(2 \mathrm{~A})$ & $55(3)$ & $38(3)$ & $90(4)$ & $-9(3)$ & $-1(3)$ & $17(3)$ \\
$\mathrm{O}(3 \mathrm{~A})$ & $100(5)$ & $37(3)$ & $78(4)$ & $12(3)$ & $-34(4)$ & $10(3)$ \\
$\mathrm{O}(4 \mathrm{~A})$ & $89(4)$ & $30(3)$ & $79(4)$ & $-8(3)$ & $-5(3)$ & $-2(3)$ \\
$\mathrm{O}(1 \mathrm{~B})$ & $130(6)$ & $73(4)$ & $51(3)$ & $3(3)$ & $33(4)$ & $48(4)$ \\
$\mathrm{O}(2 \mathrm{~B})$ & $74(4)$ & $146(6)$ & $68(4)$ & $-29(4)$ & $-12(3)$ & $62(4)$ \\
$\mathrm{O}(3 \mathrm{~B})$ & $56(4)$ & $78(4)$ & $58(3)$ & $-15(3)$ & $18(3)$ & $3(3)$ \\
$\mathrm{O}(4 \mathrm{~B})$ & $53(4)$ & $155(6)$ & $82(5)$ & $-41(4)$ & $-7(4)$ & $6(4)$ \\
$\mathrm{C}(1 \mathrm{~A})$ & $35(3)$ & $38(3)$ & $37(3)$ & $-3(3)$ & $-9(3)$ & $0(4)$ \\
$\mathrm{C}(2 \mathrm{~A})$ & $34(4)$ & $30(3)$ & $44(4)$ & $-1(3)$ & $5(3)$ & $2(3)$ \\
$\mathrm{C}(3 \mathrm{~A})$ & $43(4)$ & $42(4)$ & $47(4)$ & $7(3)$ & $-1(3)$ & $6(3)$ \\
$\mathrm{C}(4 \mathrm{~A})$ & $38(4)$ & $43(4)$ & $45(4)$ & $-1(3)$ & $1(3)$ & $6(3)$ \\
$\mathrm{C}(5 \mathrm{~A})$ & $49(4)$ & $42(4)$ & $37(4)$ & $1(3)$ & $13(4)$ & $8(3)$ \\
$\mathrm{C}(6 \mathrm{~A})$ & $40(4)$ & $30(4)$ & $43(4)$ & $1(3)$ & $8(3)$ & $4(3)$ \\
$\mathrm{C}(7 \mathrm{~A})$ & $48(4)$ & $31(4)$ & $47(4)$ & $2(3)$ & $3(3)$ & $-1(3)$ \\
$\mathrm{C}(8 \mathrm{~A})$ & $118(9)$ & $45(5)$ & $106(7)$ & $9(5)$ & $32(7)$ & $29(6)$ \\
$\mathrm{C}(9 \mathrm{~A})$ & $109(9)$ & $73(7)$ & $93(7)$ & $6(6)$ & $20(7)$ & $56(7)$ \\
$\mathrm{C}(10 \mathrm{~A})$ & $67(5)$ & $30(4)$ & $50(4)$ & $3(3)$ & $10(4)$ & $5(3)$ \\
$\mathrm{C}(11 \mathrm{~A})$ & $117(10)$ & $103(9)$ & $111(9)$ & $-23(7)$ & $-11(7)$ & $73(8)$ \\
$\mathrm{C}(12 \mathrm{~A})$ & $106(8)$ & $37(5)$ & $122(9)$ & $-13(5)$ & $10(7)$ & $22(5)$ \\
$\mathrm{C}(13 \mathrm{~A})$ & $61(5)$ & $52(5)$ & $47(4)$ & $13(4)$ & $16(4)$ & $14(4)$ \\
$\mathrm{C}(14 \mathrm{~A})$ & $115(8)$ & $84(7)$ & $74(6)$ & $21(5)$ & $50(6)$ & $17(6)$ \\
$\mathrm{C}(15 \mathrm{~A})$ & $186(12)$ & $163(12)$ & $50(6)$ & $-39(7)$ & $29(7)$ & $-57(10)$ \\
$\mathrm{C}(16 \mathrm{~A})$ & $97(7)$ & $173(11)$ & $108(8)$ & $72(8)$ & $59(6)$ & $64(8)$ \\
$\mathrm{C}(1 \mathrm{~B})$ & $43(4)$ & $37(4)$ & $39(4)$ & $2(3)$ & $2(3)$ & $-3(3)$ \\
$\mathrm{C}(2 \mathrm{~B})$ & $50(4)$ & $38(4)$ & $38(4)$ & $-12(3)$ & $-1(3)$ & $1(4)$ \\
$\mathrm{C}(3 \mathrm{~B})$ & $50(5)$ & $44(4)$ & $58(5)$ & $-1(4)$ & $23(4)$ & $-4(4)$ \\
$\mathrm{C}(4 \mathrm{~B})$ & $50(4)$ & $35(4)$ & $44(4)$ & $-1(3)$ & $6(4)$ & $-2(3)$ \\
$\mathrm{C}(5 \mathrm{~B})$ & $50(5)$ & $36(4)$ & $56(5)$ & $4(3)$ & $5(4)$ & $7(3)$ \\
& & & & & &
\end{tabular}




$\begin{array}{lcccccc}\mathrm{C}(6 \mathrm{~B}) & 45(4) & 29(3) & 37(4) & -2(3) & 1(3) & 1(3) \\ \mathrm{C}(7 \mathrm{~B}) & 46(4) & 34(4) & 57(5) & -6(3) & 11(4) & 4(3) \\ \mathrm{C}(8 \mathrm{~B}) & 106(8) & 70(6) & 111(8) & -6(6) & 66(7) & 17(6) \\ \mathrm{C}(9 \mathrm{~B}) & 65(6) & 157(11) & 116(9) & -42(8) & -14(7) & 52(7) \\ \mathrm{C}(10 \mathrm{~B}) & 62(5) & 41(4) & 70(5) & 2(4) & 15(5) & 21(4) \\ \mathrm{C}(11 \mathrm{~B}) & 68(7) & 111(9) & 140(10) & -79(8) & -2(7) & 14(6) \\ \mathrm{C}(12 \mathrm{~B}) & 77(7) & 109(9) & 101(9) & -1(7) & -18(7) & 26(6) \\ \mathrm{C}(13 \mathrm{~B}) & 66(5) & 30(4) & 86(6) & -10(4) & 19(5) & -7(4) \\ \mathrm{C}(14 \mathrm{~B}) & 138(10) & 58(6) & 217(13) & -45(7) & 99(10) & -42(7) \\ \mathrm{C}(15 \mathrm{~B}) & 160(11) & 60(6) & 104(8) & -29(6) & -25(8) & -27(7) \\ \mathrm{C}(16 \mathrm{~B}) & 136(9) & 42(5) & 137(9) & 21(6) & 4(7) & -4(6)\end{array}$


Table 5. Hydrogen coordinates $\left(\times 10^{4}\right)$ and isotropic displacement parameters $\left(\AA^{2} \times 10^{3}\right)$ for 25.

\begin{tabular}{|c|c|c|c|c|}
\hline & $\mathrm{x}$ & $\mathrm{y}$ & Z & $\mathrm{U}(\mathrm{eq})$ \\
\hline $\mathrm{H}(3 \mathrm{AA})$ & 8880 & 9220 & 5616 & 53 \\
\hline $\mathrm{H}(5 \mathrm{AA})$ & 7468 & 11850 & 5416 & 51 \\
\hline $\mathrm{H}(7 \mathrm{AA})$ & 10283 & 8995 & 4244 & 51 \\
\hline $\mathrm{H}(8 \mathrm{AA})$ & 10755 & 7158 & 4478 & 108 \\
\hline $\mathrm{H}(8 \mathrm{AB})$ & 10879 & 7070 & 5170 & 108 \\
\hline $\mathrm{H}(9 \mathrm{AA})$ & 12681 & 7967 & 5161 & 110 \\
\hline $\mathrm{H}(9 \mathrm{AB})$ & 12604 & 8003 & 4464 & 110 \\
\hline $\mathrm{H}(10 \mathrm{~A})$ & 9185 & 12394 & 4122 & 58 \\
\hline $\mathrm{H}(11 \mathrm{~A})$ & 5873 & 13184 & 4493 & 133 \\
\hline $\mathrm{H}(11 \mathrm{~B})$ & 6501 & 13552 & 3899 & 133 \\
\hline $\mathrm{H}(12 \mathrm{~A})$ & 8050 & 14260 & 4344 & 106 \\
\hline $\mathrm{H}(12 \mathrm{~B})$ & 7208 & 14107 & 4924 & 106 \\
\hline $\mathrm{H}(14 \mathrm{~A})$ & 6817 & 9093 & 6196 & 136 \\
\hline $\mathrm{H}(14 \mathrm{~B})$ & 6856 & 9498 & 6841 & 136 \\
\hline $\mathrm{H}(14 \mathrm{C})$ & 8184 & 9233 & 6517 & 136 \\
\hline $\mathrm{H}(15 \mathrm{~A})$ & 8274 & 11746 & 6516 & 199 \\
\hline $\mathrm{H}(15 \mathrm{~B})$ & 9059 & 10829 & 6709 & 199 \\
\hline $\mathrm{H}(15 \mathrm{C})$ & 7739 & 11110 & 7034 & 199 \\
\hline $\mathrm{H}(16 \mathrm{~A})$ & 6104 & 11584 & 6105 & 189 \\
\hline $\mathrm{H}(16 \mathrm{~B})$ & 5605 & 10817 & 6559 & 189 \\
\hline $\mathrm{H}(16 \mathrm{C})$ & 5561 & 10598 & 5880 & 189 \\
\hline $\mathrm{H}(3 \mathrm{BA})$ & 6121 & 8113 & 3575 & 61 \\
\hline $\mathrm{H}(5 \mathrm{BA})$ & 9470 & 7251 & 2828 & 57 \\
\hline $\mathrm{H}(7 \mathrm{BA})$ & 7027 & 10510 & 3785 & 55 \\
\hline $\mathrm{H}(8 \mathrm{BA})$ & 5204 & 10662 & 4650 & 115 \\
\hline $\mathrm{H}(8 \mathrm{BB})$ & 4335 & 9726 & 4605 & 115 \\
\hline $\mathrm{H}(9 \mathrm{BA})$ & 3607 & 10170 & 3778 & 135 \\
\hline $\mathrm{H}(9 \mathrm{BB})$ & 4449 & 11118 & 3830 & 135 \\
\hline $\mathrm{H}(10 \mathrm{~B})$ & 10891 & 9501 & 2639 & 69 \\
\hline $\mathrm{H}(11 \mathrm{C})$ & 12681 & 7362 & 2314 & 128 \\
\hline $\mathrm{H}(11 \mathrm{D})$ & 13057 & 8411 & 2126 & 128 \\
\hline
\end{tabular}




$\begin{array}{lrlll}\mathrm{H}(12 \mathrm{C}) & 13569 & 8692 & 2985 & 115 \\ \mathrm{H}(12 \mathrm{D}) & 13078 & 7671 & 3182 & 115 \\ \mathrm{H}(14 \mathrm{D}) & 5275 & 6720 & 3325 & 206 \\ \mathrm{H}(14 \mathrm{E}) & 6143 & 6522 & 3887 & 206 \\ \mathrm{H}(14 \mathrm{~F}) & 5761 & 5678 & 3463 & 206 \\ \mathrm{H}(15 \mathrm{D}) & 6311 & 6623 & 2336 & 162 \\ \mathrm{H}(15 \mathrm{E}) & 6868 & 5596 & 2461 & 162 \\ \mathrm{H}(15 \mathrm{~F}) & 7844 & 6428 & 2296 & 162 \\ \mathrm{H}(16 \mathrm{D}) & 7873 & 5202 & 3418 & 157 \\ \mathrm{H}(16 \mathrm{E}) & 8502 & 6049 & 3776 & 157 \\ \mathrm{H}(16 \mathrm{~F}) & 8996 & 5829 & 3134 & 157\end{array}$

Symmetry transformations used to generate equivalent atoms: 
Tables for crystallographic data of 26

Table 1. Crystal data and structure refinement for $\mathbf{2 6}$.

Identification code

Empirical formula

Formula weight

Temperature

Wavelength

Unit cell dimensions

Volume

Z, Calculated density

Absorption coefficient

$\mathrm{F}(000)$

Theta range for data collection

Limiting indices

Reflections collected / unique

Completeness to theta $=27.50$

Absorption correction

Refinement method

Data / restraints / parameters

Goodness-of-fit on $\mathrm{F}^{2}$

Final R indices [I $>2 \operatorname{sigma}(\mathrm{I})]$

$\mathrm{R}$ indices (all data)

Largest diff. peak and hole bischo

C24 H26 O4 Se2

536.37

296(2) K

$0.71073 \mathrm{~A}$

$\mathrm{a}=13.1015(19) \AA$ alpha $=90 \mathrm{deg}$.

$\mathrm{b}=11.423(2) \AA \quad$ beta $=91.448(12) \mathrm{deg}$.

$\mathrm{c}=32.125(5) \AA$ gamma $=90 \mathrm{deg}$.

4806.1(14) $\AA^{3}$

$8,1.483 \mathrm{Mg} / \mathrm{m}^{3}$

$3.103 \mathrm{~mm}^{-1}$

2160

2.19 to $27.50 \mathrm{deg}$.

$0<=\mathrm{h}<=17,-14<=\mathrm{k}<=0,-41<=1<=41$

$11213 / 10764[\mathrm{R}($ int $)=0.0595]$

$97.5 \%$

None

Full-matrix least-squares on $\mathrm{F}^{2}$

10764 / 48 / 618

1.044

$\mathrm{R} 1=0.0859, \mathrm{wR} 2=0.1772$

$\mathrm{R} 1=0.2073, \mathrm{wR} 2=0.2231$

0.597 and -0.718 e. $\AA^{-3}$ 
Table 2. Atomic coordinates ( $\left.\times 10^{4}\right)$ and equivalent isotropic displacement parameters $\left(\AA^{2} \mathrm{x}\right.$ $10^{3}$ ) for 26 . $U(e q)$ is defined as one third of the trace of the orthogonalized $U^{i j}$ tensor.

\begin{tabular}{|c|c|c|c|c|}
\hline & $\mathrm{x}$ & $\mathrm{y}$ & $\mathrm{z}$ & $\mathrm{U}(\mathrm{eq})$ \\
\hline $\operatorname{Se}(1 \mathrm{~A})$ & $6655(1)$ & $5844(1)$ & $387(1)$ & $53(1)$ \\
\hline $\mathrm{Se}(1 \mathrm{~B})$ & $6968(1)$ & $7483(1)$ & $810(1)$ & $57(1)$ \\
\hline $\operatorname{Se}(1 C)$ & $8322(1)$ & 12851(1) & $828(1)$ & $60(1)$ \\
\hline $\mathrm{Se}(1 \mathrm{D})$ & $8413(1)$ & $11578(1)$ & $262(1)$ & $56(1)$ \\
\hline $\mathrm{O}(1 \mathrm{~A})$ & $5558(6)$ & $3509(8)$ & $1369(3)$ & $85(3)$ \\
\hline $\mathrm{O}(2 \mathrm{~A})$ & $3979(6)$ & $7558(8)$ & $-203(3)$ & $82(3)$ \\
\hline $\mathrm{O}(1 \mathrm{~B})$ & $9214(7)$ & $5122(9)$ & $1412(3)$ & $90(3)$ \\
\hline $\mathrm{O}(2 \mathrm{~B})$ & $4251(6)$ & $7555(9)$ & $1568(3)$ & $89(3)$ \\
\hline $\mathrm{O}(1 \mathrm{C})$ & $11148(7)$ & $12187(9)$ & $1547(3)$ & $105(3)$ \\
\hline $\mathrm{O}(2 \mathrm{C})$ & $6061(7)$ & $10183(10)$ & $1203(3)$ & $110(4)$ \\
\hline $\mathrm{O}(1 \mathrm{D})$ & $9705(6)$ & $8631(8)$ & $981(3)$ & $82(3)$ \\
\hline $\mathrm{O}(2 \mathrm{D})$ & $10946(6)$ & $13669(8)$ & $-229(3)$ & $76(2)$ \\
\hline$C(1 \mathrm{~A})$ & $5245(7)$ & $5661(8)$ & $541(3)$ & $38(2)$ \\
\hline$C(2 A)$ & $5007(7)$ & 4926(9) & $865(3)$ & $48(3)$ \\
\hline$C(3 A)$ & $5753(9)$ & $4156(10)$ & $1085(4)$ & $56(3)$ \\
\hline$C(4 A)$ & $4004(7)$ & $4882(9)$ & $1025(3)$ & $48(3)$ \\
\hline$C(5 A)$ & $3228(7)$ & $5575(9)$ & $837(3)$ & $46(3)$ \\
\hline$C(6 A)$ & $2140(7)$ & $5576(9)$ & $1007(4)$ & $53(3)$ \\
\hline$C(7 A)$ & 2098(9) & 4951(16) & $1422(4)$ & $117(6)$ \\
\hline $\mathrm{C}(8 \mathrm{~A})$ & 1753(9) & $6813(10)$ & $1033(5)$ & $98(5)$ \\
\hline $\mathrm{C}(9 \mathrm{~A})$ & $1468(8)$ & 4913(12) & $696(5)$ & $85(4)$ \\
\hline$C(10 A)$ & $3479(7)$ & $6243(10)$ & $501(3)$ & $52(3)$ \\
\hline$C(11 A)$ & $4462(7)$ & $6294(8)$ & $338(3)$ & $41(2)$ \\
\hline $\mathrm{C}(12 \mathrm{~A})$ & $4612(8)$ & $6995(10)$ & $-31(3)$ & $56(3)$ \\
\hline$C(1 B)$ & $6842(7)$ & $6706(9)$ & $1342(3)$ & $48(3)$ \\
\hline$C(2 B)$ & $7615(8)$ & $6000(10)$ & $1505(4)$ & $60(3)$ \\
\hline$C(3 B)$ & $8605(9)$ & $5814(13)$ & $1300(4)$ & $69(4)$ \\
\hline$C(4 B)$ & $7465(9)$ & $5404(12)$ & $1864(4)$ & $75(4)$ \\
\hline$C(5 B)$ & $6549(10)$ & $5507(13)$ & $2087(4)$ & $75(4)$ \\
\hline$C(6 B)$ & $6363(10)$ & $4794(13)$ & $2501(4)$ & $115(6)$ \\
\hline$C(7 B)$ & $5276(13)$ & $4850(20)$ & $2660(9)$ & $122(11)$ \\
\hline $\mathrm{C}(8 \mathrm{~B})$ & $6630(20)$ & $3526(17)$ & 2399(9) & $123(11)$ \\
\hline$C(9 B)$ & 7106(19) & $5250(30)$ & $2836(8)$ & $150(14)$ \\
\hline$C(7 E)$ & $5448(17)$ & $3980(20)$ & $2430(9)$ & $110(11)$ \\
\hline $\mathrm{C}(8 \mathrm{E})$ & $7277(17)$ & $4080(30)$ & $2660(10)$ & $135(14)$ \\
\hline$C(9 E)$ & $6100(30)$ & $5720(20)$ & $2824(9)$ & $157(17)$ \\
\hline $\mathrm{C}(10 \mathrm{~B})$ & $5824(9)$ & $6226(12)$ & $1917(4)$ & $71(4)$ \\
\hline$C(11 B)$ & $5923(8)$ & $6846(10)$ & $1558(3)$ & $55(3)$ \\
\hline
\end{tabular}




$\begin{array}{lcccc}\mathrm{C}(12 B) & 5055(9) & 7562(11) & 1402(4) & 65(3) \\ \mathrm{C}(1 \mathrm{C}) & 8477(8) & 11695(9) & 1282(3) & 51(3) \\ \mathrm{C}(2 \mathrm{C}) & 9426(8) & 11631(11) & 1488(3) & 55(3) \\ \mathrm{C}(3 \mathrm{C}) & 10333(9) & 12325(10) & 1391(4) & 62(3) \\ \mathrm{C}(4 \mathrm{C}) & 9534(9) & 10792(11) & 1792(4) & 65(3) \\ \mathrm{C}(5 \mathrm{C}) & 8753(9) & 10032(11) & 1914(4) & 62(3) \\ \mathrm{C}(6 \mathrm{C}) & 8950(11) & 9147(14) & 2257(4) & 84(4) \\ \mathrm{C}(7 \mathrm{C}) & 9610(30) & 8130(20) & 2087(7) & 161(10) \\ \mathrm{C}(8 \mathrm{C}) & 9511(16) & 9714(18) & 2624(4) & 115(6) \\ \mathrm{C}(9 \mathrm{C}) & 7925(16) & 8640(20) & 2409(7) & 152(9) \\ \mathrm{C}(10 \mathrm{C}) & 7850(8) & 10157(11) & 1685(4) & 65(3) \\ \mathrm{C}(11 \mathrm{C}) & 7688(8) & 10983(11) & 1374(3) & 56(3) \\ \mathrm{C}(12 \mathrm{C}) & 6675(8) & 10959(13) & 1147(4) & 70(4) \\ \mathrm{C}(1 \mathrm{D}) & 9845(6) & 11259(9) & 343(3) & 42(2) \\ \mathrm{C}(2 \mathrm{D}) & 10161(7) & 10323(10) & 580(3) & 49(3) \\ \mathrm{C}(3 \mathrm{D}) & 9441(9) & 9415(11) & 761(4) & 59(3) \\ \mathrm{C}(4 \mathrm{D}) & 11211(7) & 10123(10) & 675(3) & 49(3) \\ \mathrm{C}(5 \mathrm{D}) & 11948(6) & 10858(9) & 508(3) & 41(2) \\ \mathrm{C}(6 \mathrm{D}) & 13088(7) & 10703(10) & 625(3) & 53(3) \\ \mathrm{C}(7 \mathrm{D}) & 13305(9) & 9633(13) & 882(6) & 95(5) \\ \mathrm{C}(8 \mathrm{D}) & 13458(9) & 11797(11) & 846(4) & 70(4) \\ \mathrm{C}(9 \mathrm{D}) & 13678(8) & 10576(15) & 220(4) & 77(4) \\ \mathrm{C}(10 \mathrm{D}) & 11617(7) & 11777(9) & 272(3) & 46(3) \\ \mathrm{C}(11 \mathrm{D}) & 10589(7) & 11991(9) & 177(3) & 46(3) \\ \mathrm{C}(12 \mathrm{D}) & 10336(9) & 13014(11) & -85(4) & 63(3) \\ \end{array}$


Table 3. Selected bond lengths $[\AA]$ and angles $[\mathrm{deg}]$ for 26.

\begin{tabular}{|c|c|}
\hline $\operatorname{Se}(1 \mathrm{~A})-\mathrm{C}(1 \mathrm{~A})$ & $1.937(9)$ \\
\hline $\operatorname{Se}(1 \mathrm{~A})-\operatorname{Se}(1 \mathrm{~B})$ & $2.3425(17)$ \\
\hline $\operatorname{Se}(1 \mathrm{~B})-\mathrm{C}(1 \mathrm{~B})$ & $1.937(10)$ \\
\hline $\operatorname{Se}(1 C)-C(1 C)$ & $1.975(11)$ \\
\hline $\operatorname{Se}(1 C)-\operatorname{Se}(1 D)$ & $2.3322(17)$ \\
\hline $\operatorname{Se}(1 \mathrm{D})-\mathrm{C}(1 \mathrm{D})$ & $1.923(9)$ \\
\hline $\mathrm{O}(1 \mathrm{~A})-\mathrm{C}(3 \mathrm{~A})$ & $1.206(12)$ \\
\hline $\mathrm{O}(2 \mathrm{~A})-\mathrm{C}(12 \mathrm{~A})$ & $1.175(12)$ \\
\hline $\mathrm{O}(1 \mathrm{~B})-\mathrm{C}(3 \mathrm{~B})$ & $1.173(14)$ \\
\hline $\mathrm{O}(2 \mathrm{~B})-\mathrm{C}(12 \mathrm{~B})$ & $1.193(12)$ \\
\hline $\mathrm{O}(1 \mathrm{C})-\mathrm{C}(3 \mathrm{C})$ & $1.179(13)$ \\
\hline $\mathrm{O}(2 \mathrm{C})-\mathrm{C}(12 \mathrm{C})$ & $1.214(14)$ \\
\hline $\mathrm{O}(1 \mathrm{D})-\mathrm{C}(3 \mathrm{D})$ & $1.187(13)$ \\
\hline $\mathrm{O}(2 \mathrm{D})-\mathrm{C}(12 \mathrm{D})$ & $1.197(12)$ \\
\hline$C(1 \mathrm{~A})-\mathrm{C}(2 \mathrm{~A})$ & $1.377(13)$ \\
\hline$C(1 \mathrm{~A})-\mathrm{C}(11 \mathrm{~A})$ & $1.403(13)$ \\
\hline $\mathrm{C}(2 \mathrm{~A})-\mathrm{C}(4 \mathrm{~A})$ & $1.424(12)$ \\
\hline $\mathrm{C}(2 \mathrm{~A})-\mathrm{C}(3 \mathrm{~A})$ & $1.482(14)$ \\
\hline$C(4 A)-C(5 A)$ & $1.412(13)$ \\
\hline$C(5 A)-C(10 A)$ & $1.368(14)$ \\
\hline$C(5 A)-C(6 A)$ & $1.540(12)$ \\
\hline$C(6 A)-C(8 A)$ & $1.504(15)$ \\
\hline$C(6 A)-C(7 A)$ & $1.516(16)$ \\
\hline$C(6 A)-C(9 A)$ & $1.518(15)$ \\
\hline$C(10 A)-C(11 A)$ & $1.404(12)$ \\
\hline $\mathrm{C}(11 \mathrm{~A})-\mathrm{C}(12 \mathrm{~A})$ & $1.449(14)$ \\
\hline$C(1 B)-C(2 B)$ & $1.388(14)$ \\
\hline$C(1 B)-C(11 B)$ & $1.414(13)$ \\
\hline$C(2 B)-C(4 B)$ & $1.358(16)$ \\
\hline $\mathrm{C}(2 \mathrm{~B})-\mathrm{C}(3 \mathrm{~B})$ & $1.485(15)$ \\
\hline$C(4 B)-C(5 B)$ & $1.417(16)$ \\
\hline $\mathrm{C}(5 \mathrm{~B})-\mathrm{C}(10 \mathrm{~B})$ & $1.360(17)$ \\
\hline$C(5 B)-C(6 B)$ & $1.582(15)$ \\
\hline$C(6 B)-C(8 E)$ & $1.525(11)$ \\
\hline$C(6 B)-C(9 B)$ & $1.526(11)$ \\
\hline $\mathrm{C}(6 \mathrm{~B})-\mathrm{C}(7 \mathrm{~B})$ & $1.526(11)$ \\
\hline$C(6 B)-C(7 E)$ & $1.527(11)$ \\
\hline$C(6 B)-C(9 E)$ & $1.528(11)$ \\
\hline$C(6 B)-C(8 B)$ & $1.528(11)$ \\
\hline $\mathrm{C}(10 \mathrm{~B})-\mathrm{C}(11 \mathrm{~B})$ & $1.364(15)$ \\
\hline $\mathrm{C}(11 \mathrm{~B})-\mathrm{C}(12 \mathrm{~B})$ & $1.478(15)$ \\
\hline$C(1 C)-C(11 C)$ & $1.355(14)$ \\
\hline$C(1 C)-C(2 C)$ & $1.396(14)$ \\
\hline
\end{tabular}




\begin{tabular}{|c|c|}
\hline$C(2 C)-C(4 C)$ & $1.371(15)$ \\
\hline$C(2 C)-C(3 C)$ & $1.468(14)$ \\
\hline$C(4 C)-C(5 C)$ & $1.405(15)$ \\
\hline$C(5 C)-C(10 C)$ & $1.386(15)$ \\
\hline$C(5 C)-C(6 C)$ & $1.513(17)$ \\
\hline$C(6 C)-C(8 C)$ & $1.517(18)$ \\
\hline$C(6 C)-C(9 C)$ & $1.552(19)$ \\
\hline$C(6 C)-C(7 C)$ & $1.56(2)$ \\
\hline$C(10 C)-C(11 C)$ & $1.386(15)$ \\
\hline$C(11 C)-C(12 C)$ & $1.498(15)$ \\
\hline$C(1 D)-C(2 D)$ & $1.372(14)$ \\
\hline$C(1 D)-C(11 D)$ & $1.399(13)$ \\
\hline$C(2 D)-C(4 D)$ & $1.419(12)$ \\
\hline$C(2 D)-C(3 D)$ & $1.528(15)$ \\
\hline$C(4 D)-C(5 D)$ & $1.396(13)$ \\
\hline$C(5 D)-C(10 D)$ & $1.361(13)$ \\
\hline $\mathrm{C}(5 \mathrm{D})-\mathrm{C}(6 \mathrm{D})$ & $1.540(12)$ \\
\hline$C(6 D)-C(7 D)$ & $1.499(15)$ \\
\hline $\mathrm{C}(6 \mathrm{D})-\mathrm{C}(8 \mathrm{D})$ & $1.510(15)$ \\
\hline$C(6 D)-C(9 D)$ & $1.537(15)$ \\
\hline $\mathrm{C}(10 \mathrm{D})-\mathrm{C}(11 \mathrm{D})$ & $1.395(12)$ \\
\hline $\mathrm{C}(11 \mathrm{D})-\mathrm{C}(12 \mathrm{D})$ & $1.473(15)$ \\
\hline$C(1 \mathrm{~A})-\mathrm{Se}(1 \mathrm{~A})-\mathrm{Se}(1 \mathrm{~B})$ & $95.3(3)$ \\
\hline $\mathrm{C}(1 \mathrm{~B})-\mathrm{Se}(1 \mathrm{~B})-\mathrm{Se}(1 \mathrm{~A})$ & $97.4(3)$ \\
\hline $\mathrm{C}(1 \mathrm{C})-\mathrm{Se}(1 \mathrm{C})-\mathrm{Se}(1 \mathrm{D})$ & $98.8(3)$ \\
\hline$C(1 D)-S e(1 D)-S e(1 C)$ & $94.7(3)$ \\
\hline$C(2 \mathrm{~A})-\mathrm{C}(1 \mathrm{~A})-\mathrm{C}(11 \mathrm{~A})$ & $119.3(8)$ \\
\hline$C(2 \mathrm{~A})-\mathrm{C}(1 \mathrm{~A})-\mathrm{Se}(1 \mathrm{~A})$ & $119.6(7)$ \\
\hline $\mathrm{C}(11 \mathrm{~A})-\mathrm{C}(1 \mathrm{~A})-\mathrm{Se}(1 \mathrm{~A})$ & $121.1(7)$ \\
\hline$C(1 \mathrm{~A})-\mathrm{C}(2 \mathrm{~A})-\mathrm{C}(4 \mathrm{~A})$ & $121.5(9)$ \\
\hline$C(1 \mathrm{~A})-\mathrm{C}(2 \mathrm{~A})-\mathrm{C}(3 \mathrm{~A})$ & $124.3(9)$ \\
\hline $\mathrm{C}(4 \mathrm{~A})-\mathrm{C}(2 \mathrm{~A})-\mathrm{C}(3 \mathrm{~A})$ & $114.2(10)$ \\
\hline $\mathrm{O}(1 \mathrm{~A})-\mathrm{C}(3 \mathrm{~A})-\mathrm{C}(2 \mathrm{~A})$ & $125.1(11)$ \\
\hline$C(5 A)-C(4 A)-C(2 A)$ & 119.1(10) \\
\hline$C(10 A)-C(5 A)-C(4 A)$ & $117.7(9)$ \\
\hline$C(10 A)-C(5 A)-C(6 A)$ & $121.5(9)$ \\
\hline$C(4 A)-C(5 A)-C(6 A)$ & $120.8(9)$ \\
\hline$C(8 A)-C(6 A)-C(7 A)$ & $112.0(12)$ \\
\hline $\mathrm{C}(8 \mathrm{~A})-\mathrm{C}(6 \mathrm{~A})-\mathrm{C}(9 \mathrm{~A})$ & $108.3(10)$ \\
\hline$C(7 A)-C(6 A)-C(9 A)$ & $108.1(10)$ \\
\hline$C(8 A)-C(6 A)-C(5 A)$ & $109.7(8)$ \\
\hline$C(7 A)-C(6 A)-C(5 A)$ & $111.5(9)$ \\
\hline$C(9 A)-C(6 A)-C(5 A)$ & $107.0(9)$ \\
\hline$C(5 A)-C(10 A)-C(11 A)$ & $123.9(10)$ \\
\hline$C(1 \mathrm{~A})-\mathrm{C}(11 \mathrm{~A})-\mathrm{C}(10 \mathrm{~A})$ & $118.1(9)$ \\
\hline
\end{tabular}




\begin{tabular}{|c|c|}
\hline$C(1 A)-C(11 A)-C(12 A)$ & $123.6(8)$ \\
\hline $\mathrm{C}(10 \mathrm{~A})-\mathrm{C}(11 \mathrm{~A})-\mathrm{C}(12 \mathrm{~A})$ & $118.3(9)$ \\
\hline $\mathrm{O}(2 \mathrm{~A})-\mathrm{C}(12 \mathrm{~A})-\mathrm{C}(11 \mathrm{~A})$ & $125.2(10)$ \\
\hline$C(2 B)-C(1 B)-C(11 B)$ & $120.2(10)$ \\
\hline$C(2 B)-C(1 B)-S e(1 B)$ & $121.5(8)$ \\
\hline$C(11 B)-C(1 B)-S e(1 B)$ & $118.3(8)$ \\
\hline $\mathrm{C}(4 \mathrm{~B})-\mathrm{C}(2 \mathrm{~B})-\mathrm{C}(1 \mathrm{~B})$ & $119.5(11)$ \\
\hline$C(4 B)-C(2 B)-C(3 B)$ & $116.9(12)$ \\
\hline$C(1 B)-C(2 B)-C(3 B)$ & $123.5(11)$ \\
\hline $\mathrm{O}(1 \mathrm{~B})-\mathrm{C}(3 \mathrm{~B})-\mathrm{C}(2 \mathrm{~B})$ & $123.7(14)$ \\
\hline$C(2 B)-C(4 B)-C(5 B)$ & $122.0(12)$ \\
\hline$C(10 B)-C(5 B)-C(4 B)$ & $116.1(12)$ \\
\hline$C(10 B)-C(5 B)-C(6 B)$ & $121.8(12)$ \\
\hline$C(4 B)-C(5 B)-C(6 B)$ & $122.1(12)$ \\
\hline$C(8 E)-C(6 B)-C(9 B)$ & $57.7(11)$ \\
\hline$C(8 E)-C(6 B)-C(7 B)$ & $129.8(16)$ \\
\hline$C(9 B)-C(6 B)-C(7 B)$ & $109.5(3)$ \\
\hline$C(8 E)-C(6 B)-C(7 E)$ & $109.5(3)$ \\
\hline$C(9 B)-C(6 B)-C(7 E)$ & $143.0(15)$ \\
\hline$C(7 B)-C(6 B)-C(7 E)$ & $48.6(11)$ \\
\hline $\mathrm{C}(8 \mathrm{E})-\mathrm{C}(6 \mathrm{~B})-\mathrm{C}(9 \mathrm{E})$ & $109.4(3)$ \\
\hline$C(9 B)-C(6 B)-C(9 E)$ & $55.6(11)$ \\
\hline$C(7 B)-C(6 B)-C(9 E)$ & $61.0(11)$ \\
\hline$C(7 E)-C(6 B)-C(9 E)$ & $109.3(3)$ \\
\hline $\mathrm{C}(8 \mathrm{E})-\mathrm{C}(6 \mathrm{~B})-\mathrm{C}(8 \mathrm{~B})$ & $51.9(11)$ \\
\hline$C(9 B)-C(6 B)-C(8 B)$ & $109.3(3)$ \\
\hline$C(7 B)-C(6 B)-C(8 B)$ & $109.3(3)$ \\
\hline$C(7 E)-C(6 B)-C(8 B)$ & $65.0(10)$ \\
\hline $\mathrm{C}(9 \mathrm{E})-\mathrm{C}(6 \mathrm{~B})-\mathrm{C}(8 \mathrm{~B})$ & $149.2(15)$ \\
\hline $\mathrm{C}(8 \mathrm{E})-\mathrm{C}(6 \mathrm{~B})-\mathrm{C}(5 \mathrm{~B})$ & $114.8(15)$ \\
\hline$C(9 B)-C(6 B)-C(5 B)$ & $107.8(14)$ \\
\hline$C(7 B)-C(6 B)-C(5 B)$ & $115.1(14)$ \\
\hline$C(7 E)-C(6 B)-C(5 B)$ & $108.8(14)$ \\
\hline$C(9 E)-C(6 B)-C(5 B)$ & $104.9(15)$ \\
\hline $\mathrm{C}(8 \mathrm{~B})-\mathrm{C}(6 \mathrm{~B})-\mathrm{C}(5 \mathrm{~B})$ & $105.5(14)$ \\
\hline$C(5 B)-C(10 B)-C(11 B)$ & $124.9(12)$ \\
\hline$C(10 B)-C(11 B)-C(1 B)$ & $117.2(11)$ \\
\hline$C(10 B)-C(11 B)-C(12 B)$ & $119.0(10)$ \\
\hline $\mathrm{C}(1 \mathrm{~B})-\mathrm{C}(11 \mathrm{~B})-\mathrm{C}(12 \mathrm{~B})$ & $123.6(10)$ \\
\hline $\mathrm{O}(2 \mathrm{~B})-\mathrm{C}(12 \mathrm{~B})-\mathrm{C}(11 \mathrm{~B})$ & $121.6(13)$ \\
\hline$C(11 C)-C(1 C)-C(2 C)$ & $122.7(10)$ \\
\hline$C(11 C)-C(1 C)-S e(1 C)$ & $119.9(8)$ \\
\hline$C(2 C)-C(1 C)-S e(1 C)$ & $117.4(8)$ \\
\hline$C(4 C)-C(2 C)-C(1 C)$ & $116.7(10)$ \\
\hline$C(4 C)-C(2 C)-C(3 C)$ & $117.3(11)$ \\
\hline$C(1 C)-C(2 C)-C(3 C)$ & $125.9(11)$ \\
\hline
\end{tabular}




\begin{tabular}{|c|c|}
\hline $\mathrm{O}(1 \mathrm{C})-\mathrm{C}(3 \mathrm{C})-\mathrm{C}(2 \mathrm{C})$ & $124.6(12)$ \\
\hline$C(2 C)-C(4 C)-C(5 C)$ & $124.6(11)$ \\
\hline$C(10 C)-C(5 C)-C(4 C)$ & $113.9(11)$ \\
\hline$C(10 C)-C(5 C)-C(6 C)$ & $125.7(11)$ \\
\hline$C(4 C)-C(5 C)-C(6 C)$ & $120.3(11)$ \\
\hline$C(5 C)-C(6 C)-C(8 C)$ & $110.5(12)$ \\
\hline$C(5 C)-C(6 C)-C(9 C)$ & $110.2(13)$ \\
\hline$C(8 C)-C(6 C)-C(9 C)$ & $108.7(14)$ \\
\hline$C(5 C)-C(6 C)-C(7 C)$ & $109.1(13)$ \\
\hline$C(8 C)-C(6 C)-C(7 C)$ & $109.1(18)$ \\
\hline$C(9 C)-C(6 C)-C(7 C)$ & $109(2)$ \\
\hline$C(5 C)-C(10 C)-C(11 C)$ & $124.5(11)$ \\
\hline$C(1 C)-C(11 C)-C(10 C)$ & $117.6(10)$ \\
\hline$C(1 C)-C(11 C)-C(12 C)$ & $125.2(11)$ \\
\hline$C(10 C)-C(11 C)-C(12 C)$ & $117.1(11)$ \\
\hline $\mathrm{O}(2 \mathrm{C})-\mathrm{C}(12 \mathrm{C})-\mathrm{C}(11 \mathrm{C})$ & $121.5(14)$ \\
\hline$C(2 D)-C(1 D)-C(11 D)$ & $118.2(8)$ \\
\hline$C(2 D)-C(1 D)-S e(1 D)$ & $120.2(7)$ \\
\hline$C(11 D)-C(1 D)-S e(1 D)$ & $121.5(8)$ \\
\hline $\mathrm{C}(1 \mathrm{D})-\mathrm{C}(2 \mathrm{D})-\mathrm{C}(4 \mathrm{D})$ & $121.5(9)$ \\
\hline$C(1 D)-C(2 D)-C(3 D)$ & $124.1(9)$ \\
\hline$C(4 D)-C(2 D)-C(3 D)$ & $114.4(10)$ \\
\hline $\mathrm{O}(1 \mathrm{D})-\mathrm{C}(3 \mathrm{D})-\mathrm{C}(2 \mathrm{D})$ & $124.4(11)$ \\
\hline$C(5 D)-C(4 D)-C(2 D)$ & $119.8(10)$ \\
\hline$C(10 D)-C(5 D)-C(4 D)$ & $117.7(9)$ \\
\hline$C(10 D)-C(5 D)-C(6 D)$ & $121.1(9)$ \\
\hline$C(4 D)-C(5 D)-C(6 D)$ & $120.9(9)$ \\
\hline$C(7 D)-C(6 D)-C(8 D)$ & $111.2(11)$ \\
\hline$C(7 D)-C(6 D)-C(9 D)$ & $107.3(12)$ \\
\hline $\mathrm{C}(8 \mathrm{D})-\mathrm{C}(6 \mathrm{D})-\mathrm{C}(9 \mathrm{D})$ & $108.3(10)$ \\
\hline$C(7 D)-C(6 D)-C(5 D)$ & 113.4(9) \\
\hline $\mathrm{C}(8 \mathrm{D})-\mathrm{C}(6 \mathrm{D})-\mathrm{C}(5 \mathrm{D})$ & $108.5(9)$ \\
\hline$C(9 D)-C(6 D)-C(5 D)$ & 108.1(9) \\
\hline$C(5 D)-C(10 D)-C(11 D)$ & $123.3(10)$ \\
\hline$C(10 D)-C(11 D)-C(1 D)$ & $119.4(10)$ \\
\hline$C(10 D)-C(11 D)-C(12 D)$ & $117.8(9)$ \\
\hline$C(1 D)-C(11 D)-C(12 D)$ & $122.8(9)$ \\
\hline $\mathrm{O}(2 \mathrm{D})-\mathrm{C}(12 \mathrm{D})-\mathrm{C}(11 \mathrm{D})$ & $125.0(11)$ \\
\hline
\end{tabular}

Symmetry transformations used to generate equivalent atoms: 
Table 4. Anisotropic displacement parameters $\left(\AA^{2} \times 10^{3}\right)$ for 26. The anisotropic displacement factor exponent takes the form: $-2 \mathrm{p}^{2}\left[\mathrm{~h}^{2} \mathrm{a}^{* 2} \mathrm{U}^{11}+\ldots+2 \mathrm{~h} \mathrm{k} \mathrm{a}^{*} \mathrm{~b}^{*} \mathrm{U}^{12}\right]$

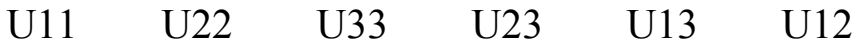

\begin{tabular}{|c|c|c|c|c|c|c|}
\hline$(1 \mathrm{~A})$ & $34(1)$ & $67(1)$ & $58(1)$ & $-7(1)$ & $14(1)$ & $0(1)$ \\
\hline $\operatorname{Se}(1 \mathrm{~B})$ & $56(1)$ & $60(1)$ & $55(1)$ & $5(1)$ & $6(1)$ & $14(1)$ \\
\hline$e(1 C)$ & $53(1)$ & $55(1)$ & $73(1)$ & 1(1) & $9(1)$ & $12(1)$ \\
\hline (1D) & $32(1)$ & $76(1)$ & $60(1)$ & $-1(1)$ & $0(1)$ & $4(1)$ \\
\hline$(1 \mathrm{~A})$ & $62(5)$ & $95(7)$ & $98(7)$ & $23(6)$ & $4(5)$ & $17(5)$ \\
\hline$(2 \mathrm{~A})$ & $51(5)$ & $118(8)$ & $78(6)$ & $50(6)$ & $8(4)$ & $15(5)$ \\
\hline$(1 \mathrm{~B})$ & $66(6)$ & $99(7)$ & $103(7)$ & $10(6)$ & $10(5)$ & $27(5)$ \\
\hline$(2 B)$ & $64(5)$ & $121(8)$ & $84(6)$ & $6(6)$ & $13(5)$ & $25(6)$ \\
\hline (1C) & $62(6)$ & $105(8)$ & $146(9)$ & $30(7)$ & $-14(6)$ & $-24(6)$ \\
\hline$(2 C)$ & $63(6)$ & 118(9) & $148(10)$ & $16(7)$ & $-26(6)$ & $-32(6)$ \\
\hline (1D) & $46(5)$ & $62(6)$ & 139(9) & $27(6)$ & $10(5)$ & $-7(4)$ \\
\hline$(2 \mathrm{D})$ & $53(5)$ & $85(6)$ & $89(6)$ & $26(5)$ & $8(4)$ & $-7(5)$ \\
\hline$(1 \mathrm{~A})$ & $38(5)$ & $27(5)$ & $49(6)$ & $-16(5)$ & $1(4)$ & $-3(4)$ \\
\hline$(2 \mathrm{~A})$ & $41(6)$ & $46(6)$ & $58(7)$ & $-6(6)$ & $2(5)$ & $4(5)$ \\
\hline$C(3 \mathrm{~A})$ & $55(7)$ & $50(7)$ & $64(8)$ & $1(6)$ & $3(6)$ & $7(6)$ \\
\hline$(4 \mathrm{~A})$ & $34(5)$ & $41(6)$ & $68(7)$ & $-6(6)$ & $20(5)$ & $-9(5)$ \\
\hline$(5 \mathrm{~A})$ & $30(5)$ & $43(6)$ & $65(7)$ & $4(6)$ & $9(5)$ & $-2(5)$ \\
\hline$C(6 \mathrm{~A})$ & $35(5)$ & $50(7)$ & $76(8)$ & $11(6)$ & $13(5)$ & $-4(5)$ \\
\hline$(7 A)$ & $56(8)$ & 193(18) & $103(12)$ & $39(13)$ & $43(8)$ & $10(10)$ \\
\hline$C(8 \mathrm{~A})$ & $61(8)$ & $56(8)$ & $180(16)$ & $-5(9)$ & $60(9)$ & $6(7)$ \\
\hline$(9 \mathrm{~A})$ & $32(6)$ & $85(10)$ & $140(13)$ & $-21(9)$ & $18(7)$ & $-14(6)$ \\
\hline$C(10 A)$ & $33(5)$ & & $68(7)$ & $-6(6)$ & $5(5)$ & $5(5)$ \\
\hline$C(11 \mathrm{~A})$ & $31(5)$ & $42(6)$ & $50(6)$ & $-5(5)$ & $13(4)$ & $0(4)$ \\
\hline$C(12 \mathrm{~A})$ & $43(6)$ & $62(8)$ & $63(7)$ & $5(6)$ & $7(6)$ & $-1(6)$ \\
\hline$(1 B)$ & $43(6)$ & $57(7)$ & $45(6)$ & $-1(6)$ & $4(5)$ & $11(5)$ \\
\hline$C(2 B)$ & $44(6)$ & $59(8)$ & $75(8)$ & $6(7)$ & $4(6)$ & $7(6)$ \\
\hline$C(3 B)$ & $51(7)$ & $95(10)$ & $60(8)$ & $-11(8)$ & $0(6)$ & $9(7)$ \\
\hline$C(4 B)$ & $52(7)$ & $93(10)$ & $81(9)$ & $21(8)$ & $-12(7)$ & $-1(7)$ \\
\hline $\mathrm{C}(5 \mathrm{~B})$ & $77(9)$ & $93(11)$ & $57(8)$ & $13(8)$ & $8(7)$ & $-13(8)$ \\
\hline $\mathrm{C}(6 \mathrm{~B})$ & $109(12)$ & $137(15)$ & $98(1$ & $38(12)$ & -1 & -10 \\
\hline $\mathrm{C}(7 \mathrm{~B})$ & $122(14)$ & $126(14)$ & 118 & $19(9)$ & $15(9)$ & $-9(9)$ \\
\hline $\mathrm{C}(8 \mathrm{~B})$ & $131(14)$ & $122(14)$ & $117(14)$ & $12(9)$ & $5(9)$ & $-5(9)$ \\
\hline $\mathrm{C}(9 \mathrm{~B})$ & $151(16)$ & $156(16)$ & $143(16)$ & $9(10)$ & $0(10)$ & $-3(10$ \\
\hline$C(7 E)$ & $109(13)$ & 111(14) & 111(14) & $16(9)$ & $8(9)$ & $-11(9)$ \\
\hline $\mathrm{C}(8 \mathrm{E})$ & $130(16)$ & $139(16)$ & $135(16)$ & $15(10)$ & $1(10)$ & $1(10)$ \\
\hline$C(9 E)$ & 159(19) & $160(19)$ & 151(19) & $6(10)$ & $1(10)$ & $0(10)$ \\
\hline $\mathrm{C}(10 \mathrm{~B})$ & $55(7)$ & $106(11)$ & $52(7)$ & $11(7)$ & $7(6)$ & $-10(7)$ \\
\hline$C(11 B)$ & $47(6)$ & $70(8)$ & $48(6)$ & $-4(6)$ & $7(5)$ & $-5(6)$ \\
\hline
\end{tabular}




$\begin{array}{lllllll}\mathrm{C}(12 \mathrm{~B}) & 54(7) & 67(8) & 75(9) & -4(8) & 15(6) & 12(6) \\ \mathrm{C}(1 \mathrm{C}) & 57(7) & 44(6) & 51(6) & -9(5) & 7(5) & -7(5) \\ \mathrm{C}(2 \mathrm{C}) & 49(6) & 67(8) & 49(7) & -13(6) & 11(5) & -11(6) \\ \mathrm{C}(3 \mathrm{C}) & 57(7) & 53(8) & 75(8) & 11(7) & 4(6) & -12(6) \\ \mathrm{C}(4 \mathrm{C}) & 61(8) & 74(9) & 59(8) & 0(7) & -6(6) & -2(7) \\ \mathrm{C}(5 \mathrm{C}) & 70(8) & 63(8) & 54(7) & -6(6) & 12(6) & -6(7) \\ \mathrm{C}(6 \mathrm{C}) & 99(10) & 91(11) & 62(9) & 0(8) & -7(8) & -38(9) \\ \mathrm{C}(7 \mathrm{C}) & 250(30) & 121(17) & 112(19) & 12(15) & -4(17) & 60(20) \\ \mathrm{C}(8 \mathrm{C}) & 155(16) & 139(17) & 49(8) & 36(10) & -9(10) & -50(15) \\ \mathrm{C}(9 \mathrm{C}) & 162(19) & 160(20) & 134(16) & 64(19) & -2(16) & -84(17) \\ \mathrm{C}(10 \mathrm{C}) & 40(6) & 66(8) & 91(10) & -11(8) & 14(6) & -15(6) \\ \mathrm{C}(11 \mathrm{C}) & 45(6) & 66(8) & 58(7) & 10(6) & 8(5) & -6(6) \\ \mathrm{C}(12 \mathrm{C}) & 38(6) & 74(9) & 96(10) & -16(9) & -3(6) & 3(6) \\ \mathrm{C}(1 \mathrm{D}) & 29(5) & 59(7) & 38(5) & -6(5) & 3(4) & 5(5) \\ \mathrm{C}(2 \mathrm{D}) & 28(5) & 60(7) & 58(7) & -8(6) & 10(5) & -4(5) \\ \mathrm{C}(3 \mathrm{D}) & 42(6) & 62(8) & 75(9) & -8(7) & 18(6) & 1(6) \\ \mathrm{C}(4 \mathrm{D}) & 33(5) & 52(7) & 62(7) & 7(6) & 1(5) & 1(5) \\ \mathrm{C}(5 \mathrm{D}) & 30(5) & 43(6) & 50(6) & -15(5) & 6(4) & 4(5) \\ \mathrm{C}(6 \mathrm{D}) & 33(5) & 59(7) & 66(7) & 10(6) & -4(5) & 2(5) \\ \mathrm{C}(7 \mathrm{D}) & 37(7) & 85(11) & 161(17) & 51(11) & -22(8) & -3(7) \\ \mathrm{C}(8 \mathrm{D}) & 52(7) & 81(10) & 75(9) & -17(9) & -7(7) & -9(7) \\ \mathrm{C}(9 \mathrm{D}) & 31(6) & 113(12) & 89(10) & -22(10) & 16(6) & -3(7) \\ \mathrm{C}(10 \mathrm{D}) & 37(5) & 51(7) & 49(6) & -1(5) & 0(5) & 2(5) \\ \mathrm{C}(11 \mathrm{D}) & 28(5) & 59(7) & 51(6) & -11(6) & 5(4) & 4(5) \\ \mathrm{C}(12 \mathrm{D}) & 50(7) & 62(8) & 76(9) & 21(7) & -3(6) & -2(6)\end{array}$


Table 5. Hydrogen coordinates ( $\left.\times 10^{4}\right)$ and isotropic displacement parameters $\left(\AA^{2} \times 10^{3}\right)$ for 26.

\begin{tabular}{|c|c|c|c|c|}
\hline & $\mathrm{x}$ & $\mathrm{y}$ & $\mathrm{z}$ & $\mathrm{U}(\mathrm{eq})$ \\
\hline $\mathrm{H}(3 \mathrm{AA})$ & 6425 & 4173 & 998 & $70(30)$ \\
\hline $\mathrm{H}(4 \mathrm{AA})$ & 3862 & 4404 & 1250 & $30(20)$ \\
\hline $\mathrm{H}(7 \mathrm{AA})$ & 1398 & 4850 & 1497 & 175 \\
\hline $\mathrm{H}(7 \mathrm{AB})$ & 2449 & 5407 & 1632 & 175 \\
\hline $\mathrm{H}(7 \mathrm{AC})$ & 2419 & 4198 & 1401 & 175 \\
\hline $\mathrm{H}(8 \mathrm{AA})$ & 1059 & 6807 & 1121 & 147 \\
\hline $\mathrm{H}(8 \mathrm{AB})$ & 1785 & 7176 & 764 & 147 \\
\hline $\mathrm{H}(8 \mathrm{AC})$ & 2168 & 7245 & 1229 & 147 \\
\hline $\mathrm{H}(9 \mathrm{AA})$ & 765 & 5007 & 766 & 128 \\
\hline $\mathrm{H}(9 \mathrm{AB})$ & 1644 & 4097 & 703 & 128 \\
\hline $\mathrm{H}(9 \mathrm{AC})$ & 1571 & 5215 & 421 & 128 \\
\hline $\mathrm{H}(10 \mathrm{~A})$ & 2967 & 6690 & 373 & $80(40)$ \\
\hline $\mathrm{H}(12 \mathrm{~A})$ & 5262 & 7001 & -141 & $70(30)$ \\
\hline $\mathrm{H}(3 \mathrm{BA})$ & 8750 & 6271 & 1069 & $110(60)$ \\
\hline $\mathrm{H}(4 \mathrm{BA})$ & 7979 & 4914 & 1967 & $30(20)$ \\
\hline $\mathrm{H}(7 \mathrm{BA})$ & 5150 & 5614 & 2771 & 183 \\
\hline $\mathrm{H}(7 \mathrm{BB})$ & 5195 & 4273 & 2874 & 183 \\
\hline $\mathrm{H}(7 \mathrm{BC})$ & 4801 & 4695 & 2434 & 183 \\
\hline $\mathrm{H}(8 \mathrm{BA})$ & 6642 & 3070 & 2650 & 184 \\
\hline $\mathrm{H}(8 \mathrm{BB})$ & 7290 & 3498 & 2276 & 184 \\
\hline $\mathrm{H}(8 \mathrm{BC})$ & 6128 & 3213 & 2207 & 184 \\
\hline $\mathrm{H}(9 \mathrm{BA})$ & 7734 & 5478 & 2710 & 226 \\
\hline $\mathrm{H}(9 \mathrm{BB})$ & 7239 & 4652 & 3038 & 226 \\
\hline $\mathrm{H}(9 \mathrm{BC})$ & 6813 & 5921 & 2969 & 226 \\
\hline $\mathrm{H}(7 \mathrm{EA})$ & 5396 & 3460 & 2662 & 165 \\
\hline $\mathrm{H}(7 \mathrm{~EB})$ & 5537 & 3541 & 2179 & 165 \\
\hline $\mathrm{H}(7 \mathrm{EC})$ & 4836 & 4443 & 2403 & 165 \\
\hline $\mathrm{H}(8 \mathrm{EA})$ & 7174 & 3857 & 2944 & 202 \\
\hline $\mathrm{H}(8 \mathrm{~EB})$ & 7885 & 4546 & 2645 & 202 \\
\hline $\mathrm{H}(8 \mathrm{EC})$ & 7347 & 3393 & 2492 & 202 \\
\hline $\mathrm{H}(9 \mathrm{EA})$ & 6114 & 5374 & 3096 & 235 \\
\hline $\mathrm{H}(9 \mathrm{~EB})$ & 5428 & 6024 & 2762 & 235 \\
\hline $\mathrm{H}(9 \mathrm{EC})$ & 6587 & 6346 & 2815 & 235 \\
\hline $\mathrm{H}(10 \mathrm{~B})$ & 5214 & 6300 & 2057 & $120(50)$ \\
\hline $\mathrm{H}(12 \mathrm{~B})$ & 5137 & 8029 & 1168 & $100(50)$ \\
\hline $\mathrm{H}(3 \mathrm{CA})$ & 10259 & 12914 & 1193 & $70(40)$ \\
\hline $\mathrm{H}(4 \mathrm{CA})$ & 10169 & 10721 & 1925 & $60(30)$ \\
\hline $\mathrm{H}(7 \mathrm{CA})$ & 10301 & 8399 & 2056 & $250(180)$ \\
\hline $\mathrm{H}(7 \mathrm{CB})$ & 9338 & 7884 & 1821 & $100(60)$ \\
\hline
\end{tabular}




\begin{tabular}{lcccc}
$\mathrm{H}(7 \mathrm{CC})$ & 9606 & 7486 & 2278 & $600(400)$ \\
$\mathrm{H}(8 \mathrm{CA})$ & 10185 & 9932 & 2543 & $100(50)$ \\
$\mathrm{H}(8 \mathrm{CB})$ & 9556 & 9170 & 2851 & $100(40)$ \\
$\mathrm{H}(8 \mathrm{CC})$ & 9146 & 10399 & 2709 & $220(120)$ \\
$\mathrm{H}(9 \mathrm{CA})$ & 7482 & 8482 & 2174 & $1700(1400)$ \\
$\mathrm{H}(9 \mathrm{CB})$ & 7607 & 9198 & 2588 & $100(70)$ \\
$\mathrm{H}(9 \mathrm{CC})$ & 8054 & 7928 & 2560 & $90(50)$ \\
$\mathrm{H}(10 \mathrm{C})$ & 7315 & 9652 & 1744 & $30(20)$ \\
$\mathrm{H}(12 \mathrm{C})$ & 6514 & 11552 & 958 & $50(30)$ \\
$\mathrm{H}(3 \mathrm{DA})$ & 8748 & 9482 & 695 & $400(200)$ \\
$\mathrm{H}(4 \mathrm{DA})$ & 11407 & 9503 & 847 & $70(40)$ \\
$\mathrm{H}(7 \mathrm{DA})$ & 12881 & 9000 & 784 & $260(120)$ \\
$\mathrm{H}(7 \mathrm{DB})$ & 13162 & 9793 & 1168 & $210(120)$ \\
$\mathrm{H}(7 \mathrm{DC})$ & 14010 & 9420 & 859 & $130(60)$ \\
$\mathrm{H}(8 \mathrm{DA})$ & 14151 & 11687 & 942 & $80(40)$ \\
$\mathrm{H}(8 \mathrm{DB})$ & 13034 & 11951 & 1079 & $70(40)$ \\
$\mathrm{H}(8 \mathrm{DC})$ & 13423 & 12447 & 657 & $80(40)$ \\
$\mathrm{H}(9 \mathrm{DA})$ & 13473 & 9868 & 81 & $110(60)$ \\
$\mathrm{H}(9 \mathrm{DB})$ & 14397 & 10546 & 284 & $70(30)$ \\
$\mathrm{H}(9 \mathrm{DC})$ & 13534 & 11234 & 42 & $210(100)$ \\
$\mathrm{H}(10 \mathrm{D})$ & 12101 & 12289 & 168 & $50(30)$ \\
$\mathrm{H}(12 \mathrm{D})$ & 9649 & 13163 & -142 & $410(190)$ \\
& & & & \\
\hline
\end{tabular}




\section{Tables for crystallographic data of 27}

Table 1. Crystal data and structure refinement for 27.

Identification code

Empirical formula

Formula weight

Temperature

Wavelength

Crystal system

Space group

Unit cell dimensions

Volume

Z

Density (calculated)

Absorption coefficient

$\mathrm{F}(000)$

Crystal size

Theta range for data collection

Index ranges

Reflections collected

Independent reflections

Completeness to theta $=28.31^{\circ}$

Absorption correction

Max. and min. transmission

Refinement method

Data / restraints / parameters

Goodness-of-fit on $\mathrm{F}^{2}$

Final $\mathrm{R}$ indices [I $>2 \operatorname{sigma}(\mathrm{I})]$

$\mathrm{R}$ indices (all data)

Extinction coefficient

Largest diff. peak and hole chosephm

C18 H18 O2 Se

345.28

93(2) K

$0.71073 \AA$

Monoclinic

$\mathrm{P} 2(1) / \mathrm{c}$

$\mathrm{a}=9.624(3) \AA \quad \mathrm{a}=90^{\circ}$.

$\mathrm{b}=13.494(4) \AA$

$\mathrm{b}=106.284(5)^{\circ}$.

$\mathrm{c}=12.323(4) \AA$ $\mathrm{g}=90^{\circ}$.
$1536.2(8) \AA^{3}$

4

$1.493 \mathrm{Mg} / \mathrm{m}^{3}$

$2.446 \mathrm{~mm}^{-1}$

704

$0.5 \times 0.65 \times 0.75 \mathrm{~mm}^{3}$

2.20 to $28.31^{\circ}$.

$-12<=\mathrm{h}<=12,-16<=\mathrm{k}<=17,-16<=1<=16$

11382

$3761[\mathrm{R}($ int $)=0.0448]$

$98.2 \%$

Sadabs

1.0000 and 0.6420

Full-matrix least-squares on $\mathrm{F}^{2}$

$3761 / 0 / 212$

0.982

$\mathrm{R} 1=0.0261, \mathrm{wR} 2=0.0561$

$\mathrm{R} 1=0.0391, \mathrm{wR} 2=0.0589$

$0.0061(7)$

0.376 and -0.303 e. $\AA^{-3}$ 
Table 2. Atomic coordinates $\left(\times 10^{4}\right)$ and equivalent isotropic displacement parameters $\left(\AA^{2} \times 10^{3}\right)$ for 27. $\mathrm{U}(\mathrm{eq})$ is defined as one third of the trace of the orthogonalized $\mathrm{Uij}^{\mathrm{ij}}$ tensor.

\begin{tabular}{|c|c|c|c|c|}
\hline & $\mathrm{x}$ & $\mathrm{y}$ & $\mathrm{z}$ & $\mathrm{U}(\mathrm{eq})$ \\
\hline $\mathrm{Se}$ & $4480(1)$ & $1054(1)$ & $6353(1)$ & $17(1)$ \\
\hline $\mathrm{O}(2)$ & $5420(2)$ & $3089(1)$ & $9279(1)$ & $31(1)$ \\
\hline $\mathrm{O}(6)$ & $8579(2)$ & $-7(1)$ & $5923(1)$ & $28(1)$ \\
\hline $\mathrm{C}(1)$ & $6371(2)$ & $1388(1)$ & $7321(1)$ & $15(1)$ \\
\hline $\mathrm{C}(2)$ & $6537(2)$ & 2064(1) & $8211(1)$ & $16(1)$ \\
\hline$C(21)$ & $5306(2)$ & $2593(2)$ & $8443(2)$ & $22(1)$ \\
\hline $\mathrm{C}(3)$ & $7909(2)$ & $2276(1)$ & $8905(1)$ & $14(1)$ \\
\hline $\mathrm{C}(4)$ & $9151(2)$ & 1841(1) & $8765(1)$ & $14(1)$ \\
\hline$C(41)$ & $10652(2)$ & $2047(1)$ & $9557(2)$ & $18(1)$ \\
\hline$C(42)$ & $10612(2)$ & $2847(1)$ & $10424(2)$ & $22(1)$ \\
\hline$C(43)$ & $11673(2)$ & $2376(2)$ & $8873(2)$ & $26(1)$ \\
\hline$C(44)$ & $11246(2)$ & $1093(2)$ & $10203(2)$ & $31(1)$ \\
\hline $\mathrm{C}(5)$ & $8965(2)$ & $1198(1)$ & $7852(2)$ & $16(1)$ \\
\hline$C(6)$ & $7611(2)$ & $964(1)$ & $7139(2)$ & $17(1)$ \\
\hline$C(61)$ & $7540(2)$ & $258(1)$ & $6197(2)$ & $25(1)$ \\
\hline$C(7)$ & $3857(2)$ & $177(1)$ & $7350(1)$ & $15(1)$ \\
\hline $\mathrm{C}(8)$ & $2403(2)$ & $-84(1)$ & $7034(2)$ & $17(1)$ \\
\hline $\mathrm{C}(9)$ & $1896(2)$ & $-754(1)$ & $7681(2)$ & $21(1)$ \\
\hline$C(10)$ & $2835(2)$ & $-1168(1)$ & $8639(2)$ & $26(1)$ \\
\hline $\mathrm{C}(11)$ & $4265(2)$ & $-909(1)$ & $8946(2)$ & $27(1)$ \\
\hline$C(12)$ & 4793(2) & $-234(1)$ & $8309(2)$ & $21(1)$ \\
\hline
\end{tabular}


Table 3. Bond lengths $[\AA]$ and angles $\left[{ }^{\circ}\right]$ for 27.

\begin{tabular}{lc}
\hline $\mathrm{Se}-\mathrm{C}(7)$ & $1.9188(17)$ \\
$\mathrm{Se}-\mathrm{C}(1)$ & $1.9290(18)$ \\
$\mathrm{O}(2)-\mathrm{C}(21)$ & $1.208(2)$ \\
$\mathrm{O}(6)-\mathrm{C}(61)$ & $1.197(2)$ \\
$\mathrm{C}(1)-\mathrm{C}(6)$ & $1.397(2)$ \\
$\mathrm{C}(1)-\mathrm{C}(2)$ & $1.401(2)$ \\
$\mathrm{C}(2)-\mathrm{C}(3)$ & $1.387(2)$ \\
$\mathrm{C}(2)-\mathrm{C}(21)$ & $1.478(2)$ \\
$\mathrm{C}(3)-\mathrm{C}(4)$ & $1.386(2)$ \\
$\mathrm{C}(4)-\mathrm{C}(5)$ & $1.392(2)$ \\
$\mathrm{C}(4)-\mathrm{C}(41)$ & $1.525(2)$ \\
$\mathrm{C}(41)-\mathrm{C}(42)$ & $1.527(2)$ \\
$\mathrm{C}(41)-\mathrm{C}(43)$ & $1.529(2)$ \\
$\mathrm{C}(41)-\mathrm{C}(44)$ & $1.537(3)$ \\
$\mathrm{C}(5)-\mathrm{C}(6)$ & $1.389(2)$ \\
$\mathrm{C}(6)-\mathrm{C}(61)$ & $1.488(2)$ \\
$\mathrm{C}(7)-\mathrm{C}(12)$ & $1.386(2)$ \\
$\mathrm{C}(7)-\mathrm{C}(8)$ & $1.388(2)$ \\
$\mathrm{C}(8)-\mathrm{C}(9)$ & $1.382(2)$ \\
$\mathrm{C}(9)-\mathrm{C}(10)$ & $1.387(3)$ \\
$\mathrm{C}(10)-\mathrm{C}(11)$ & $1.366(3)$ \\
$\mathrm{C}(11)-\mathrm{C}(12)$ & $1.388(3)$ \\
$\mathrm{C}(7)-\mathrm{Se}-\mathrm{C}(1)$ & $99.10(8)$ \\
$\mathrm{C}(6)-\mathrm{C}(1)-\mathrm{C}(2)$ & $118.50(16)$ \\
$\mathrm{C}(6)-\mathrm{C}(1)-\mathrm{Se}$ & $120.40(13)$ \\
$\mathrm{C}(2)-\mathrm{C}(1)-\mathrm{Se}$ & $121.10(13)$ \\
$\mathrm{C}(3)-\mathrm{C}(2)-\mathrm{C}(1)$ & $119.83(15)$ \\
$\mathrm{C}(3)-\mathrm{C}(2)-\mathrm{C}(21)$ & $117.17(16)$ \\
$\mathrm{C}(1)-\mathrm{C}(2)-\mathrm{C}(21)$ & $122.98(16)$ \\
$\mathrm{O}(2)-\mathrm{C}(21)-\mathrm{C}(2)$ & $123.16(18)$ \\
$\mathrm{C}(4)-\mathrm{C}(3)-\mathrm{C}(2)$ & $122.73(16)$ \\
$\mathrm{C}(3)-\mathrm{C}(4)-\mathrm{C}(5)$ & $116.44(16)$ \\
$\mathrm{C}(3)-\mathrm{C}(4)-\mathrm{C}(41)$ & $122.53(15)$ \\
&
\end{tabular}




$\begin{array}{ll}\mathrm{C}(5)-\mathrm{C}(4)-\mathrm{C}(41) & 121.03(15) \\ \mathrm{C}(4)-\mathrm{C}(41)-\mathrm{C}(42) & 111.85(14) \\ \mathrm{C}(4)-\mathrm{C}(41)-\mathrm{C}(43) & 109.84(14) \\ \mathrm{C}(42)-\mathrm{C}(41)-\mathrm{C}(43) & 109.09(15) \\ \mathrm{C}(4)-\mathrm{C}(41)-\mathrm{C}(44) & 109.06(15) \\ \mathrm{C}(42)-\mathrm{C}(41)-\mathrm{C}(44) & 108.00(16) \\ \mathrm{C}(43)-\mathrm{C}(41)-\mathrm{C}(44) & 108.93(16) \\ \mathrm{C}(6)-\mathrm{C}(5)-\mathrm{C}(4) & 122.56(16) \\ \mathrm{C}(5)-\mathrm{C}(6)-\mathrm{C}(1) & 119.88(16) \\ \mathrm{C}(5)-\mathrm{C}(6)-\mathrm{C}(61) & 117.93(16) \\ \mathrm{C}(1)-\mathrm{C}(6)-\mathrm{C}(61) & 122.19(16) \\ \mathrm{O}(6)-\mathrm{C}(61)-\mathrm{C}(6) & 123.56(18) \\ \mathrm{C}(12)-\mathrm{C}(7)-\mathrm{C}(8) & 120.07(16) \\ \mathrm{C}(12)-\mathrm{C}(7)-\mathrm{Se} & 123.23(14) \\ \mathrm{C}(8)-\mathrm{C}(7)-\mathrm{Se} & 116.60(13) \\ \mathrm{C}(9)-\mathrm{C}(8)-\mathrm{C}(7) & 119.67(17) \\ \mathrm{C}(8)-\mathrm{C}(9)-\mathrm{C}(10) & 120.22(18) \\ \mathrm{C}(11)-\mathrm{C}(10)-\mathrm{C}(9) & 119.88(18) \\ \mathrm{C}(10)-\mathrm{C}(11)-\mathrm{C}(12) & 120.74(19) \\ \mathrm{C}(7)-\mathrm{C}(12)-\mathrm{C}(11) & 119.43(18)\end{array}$

Symmetry transformations used to generate equivalent atoms: 
Table 4. Anisotropic displacement parameters $\left(\AA^{2} \times 10^{3}\right)$ for 27. The anisotropic displacement factor exponent takes the form: $-2 p^{2}\left[h^{2} a^{* 2} U^{11}+\ldots+2 h k^{*} b^{*} U^{12}\right]$

\begin{tabular}{lcccccc}
\hline & $\mathrm{U} 11$ & $\mathrm{U}^{22}$ & $\mathrm{U} 33$ & $\mathrm{U}^{23}$ & $\mathrm{U} 13$ & $\mathrm{U} 12$ \\
\hline $\mathrm{Se}$ & $11(1)$ & $23(1)$ & $15(1)$ & $4(1)$ & $0(1)$ & $-2(1)$ \\
$\mathrm{O}(2)$ & $23(1)$ & $40(1)$ & $31(1)$ & $-10(1)$ & $12(1)$ & $4(1)$ \\
$\mathrm{O}(6)$ & $25(1)$ & $31(1)$ & $28(1)$ & $-10(1)$ & $8(1)$ & $4(1)$ \\
$\mathrm{C}(1)$ & $10(1)$ & $19(1)$ & $15(1)$ & $3(1)$ & $1(1)$ & $-2(1)$ \\
$\mathrm{C}(2)$ & $14(1)$ & $17(1)$ & $17(1)$ & $4(1)$ & $6(1)$ & $0(1)$ \\
$\mathrm{C}(21)$ & $15(1)$ & $27(1)$ & $26(1)$ & $0(1)$ & $8(1)$ & $1(1)$ \\
$\mathrm{C}(3)$ & $17(1)$ & $13(1)$ & $14(1)$ & $1(1)$ & $5(1)$ & $-2(1)$ \\
$\mathrm{C}(4)$ & $14(1)$ & $13(1)$ & $16(1)$ & $2(1)$ & $4(1)$ & $-2(1)$ \\
$\mathrm{C}(41)$ & $13(1)$ & $18(1)$ & $20(1)$ & $-2(1)$ & $2(1)$ & $-2(1)$ \\
$\mathrm{C}(42)$ & $16(1)$ & $27(1)$ & $22(1)$ & $-8(1)$ & $3(1)$ & $-7(1)$ \\
$\mathrm{C}(43)$ & $16(1)$ & $33(1)$ & $30(1)$ & $-9(1)$ & $8(1)$ & $-8(1)$ \\
$\mathrm{C}(44)$ & $23(1)$ & $25(1)$ & $34(1)$ & $1(1)$ & $-10(1)$ & $-1(1)$ \\
$\mathrm{C}(5)$ & $13(1)$ & $17(1)$ & $19(1)$ & $0(1)$ & $5(1)$ & $1(1)$ \\
$\mathrm{C}(6)$ & $17(1)$ & $15(1)$ & $17(1)$ & $-1(1)$ & $4(1)$ & $-1(1)$ \\
$\mathrm{C}(61)$ & $19(1)$ & $27(1)$ & $24(1)$ & $-9(1)$ & $0(1)$ & $1(1)$ \\
$\mathrm{C}(7)$ & $18(1)$ & $15(1)$ & $14(1)$ & $0(1)$ & $6(1)$ & $0(1)$ \\
$\mathrm{C}(8)$ & $15(1)$ & $16(1)$ & $19(1)$ & $-2(1)$ & $2(1)$ & $2(1)$ \\
$\mathrm{C}(9)$ & $19(1)$ & $20(1)$ & $28(1)$ & $-5(1)$ & $10(1)$ & $-4(1)$ \\
$\mathrm{C}(10)$ & $35(1)$ & $22(1)$ & $23(1)$ & $2(1)$ & $14(1)$ & $-6(1)$ \\
$\mathrm{C}(11)$ & $33(1)$ & $25(1)$ & $19(1)$ & $6(1)$ & $1(1)$ & $-2(1)$ \\
$\mathrm{C}(12)$ & $17(1)$ & $25(1)$ & $19(1)$ & $2(1)$ & $0(1)$ & $-2(1)$ \\
& & & & & & \\
\hline
\end{tabular}


Table 5. Hydrogen coordinates ( $\left.\times 10^{4}\right)$ and isotropic displacement parameters $\left(\AA^{2} \times 10^{3}\right)$ for 27.

\begin{tabular}{|c|c|c|c|c|}
\hline & $\mathrm{x}$ & $\mathrm{y}$ & $\mathrm{z}$ & $\mathrm{U}(\mathrm{eq})$ \\
\hline $\mathrm{H}(21 \mathrm{~A})$ & 4381 & 2540 & 7908 & $26(6)$ \\
\hline $\mathrm{H}(3 \mathrm{~A})$ & 8001 & 2739 & 9502 & $13(5)$ \\
\hline $\mathrm{H}(42 \mathrm{~A})$ & 10000 & 2625 & 10893 & $24(5)$ \\
\hline $\mathrm{H}(42 \mathrm{~B})$ & 10214 & 3460 & 10031 & $28(6)$ \\
\hline $\mathrm{H}(42 \mathrm{C})$ & 11595 & 2970 & 10905 & 19(5) \\
\hline $\mathrm{H}(43 \mathrm{~A})$ & 11300 & 2982 & 8456 & $42(7)$ \\
\hline $\mathrm{H}(43 \mathrm{~B})$ & 11739 & 1852 & 8339 & $27(5)$ \\
\hline $\mathrm{H}(43 \mathrm{C})$ & 12637 & 2504 & 9387 & $32(6)$ \\
\hline $\mathrm{H}(44 \mathrm{~A})$ & 10596 & 877 & 10643 & $38(6)$ \\
\hline $\mathrm{H}(44 \mathrm{~B})$ & 12211 & 1221 & 10713 & $52(8)$ \\
\hline $\mathrm{H}(44 \mathrm{C})$ & 11309 & 573 & 9663 & $31(6)$ \\
\hline $\mathrm{H}(5 \mathrm{~A})$ & 9797 & 907 & 7712 & $22(5)$ \\
\hline $\mathrm{H}(61 \mathrm{~A})$ & 6620 & 5 & 5791 & $46(7)$ \\
\hline $\mathrm{H}(8 \mathrm{~A})$ & 1760 & 197 & 6376 & $21(5)$ \\
\hline $\mathrm{H}(9 \mathrm{~A})$ & 901 & -931 & 7469 & $18(5)$ \\
\hline $\mathrm{H}(10 \mathrm{~A})$ & 2485 & -1631 & 9080 & $30(6)$ \\
\hline $\mathrm{H}(11 \mathrm{~A})$ & 4905 & -1194 & 9602 & $25(6)$ \\
\hline $\mathrm{H}(12 \mathrm{~A})$ & 5788 & -56 & 8529 & $28(6)$ \\
\hline
\end{tabular}

Symmetry transformations used to generate equivalent atoms: 


\section{Tables for crystallographic data of 32}

Table 1. Crystal data and structure refinement for 32.

Identification code

Empirical formula

Formula weight

Temperature

Wavelength

Crystal system, space group

Unit cell dimensions

Volume

Z, Calculated density

Absorption coefficient

$\mathrm{F}(000)$

Crystal size

Theta range for data collection

Limiting indices

Reflections collected / unique

Completeness to theta $=26.73$

Absorption correction

Max. and min. transmission

Refinement method

Data / restraints / parameters

Goodness-of-fit on $\mathrm{F}^{2}$

Final $\mathrm{R}$ indices $[\mathrm{I}>2 \operatorname{sigma}(\mathrm{I})]$

$\mathrm{R}$ indices (all data)

Largest diff. peak and hole
0371

C26 H28 N2 O3 Se

495.46

293(2) K

$0.71073 \mathrm{~A}$

Triclinic, $\mathrm{p}-1$

$\mathrm{a}=9.7598(7) \AA \quad$ alpha $=88.969(11) \mathrm{deg}$.

$\mathrm{b}=10.5955(9) \AA \quad$ beta $=81.747(10) \mathrm{deg}$.

$\mathrm{c}=12.6066(12) \AA$ gamma $=70.167(9) \mathrm{deg}$.

1212.96(18) $\AA^{3}$

2, $1.357 \mathrm{Mg} / \mathrm{m}^{3}$

$1.577 \mathrm{~mm}^{-1}$

512

$0.33 \times 0.27 \times 0.13 \mathrm{~mm}$

2.66 to $26.73 \mathrm{deg}$.

$-12<=\mathrm{h}<=12,-13<=\mathrm{k}<=13,-15<=\mathrm{l}<=15$

$19069 / 4810[\mathrm{R}($ int $)=0.1385]$

$93.1 \%$

Analytical

0.81273 and 0.66189

Full-matrix least-squares on $\mathrm{F}^{2}$

4810 / 0 / 293

0.804

$\mathrm{R} 1=0.0379, \mathrm{wR} 2=0.0693$

$\mathrm{R} 1=0.0787, \mathrm{wR} 2=0.0778$

0.754 and -0.485 e. $\AA^{-3}$ 
Table 2. Atomic coordinates $\left(\times 10^{4}\right)$ and equivalent isotropic displacement parameters $\left(\AA^{2} \times 10^{3}\right)$ for 32. $U(e q)$ is defined as one third of the trace of the orthogonalized $U^{i j}$ tensor.

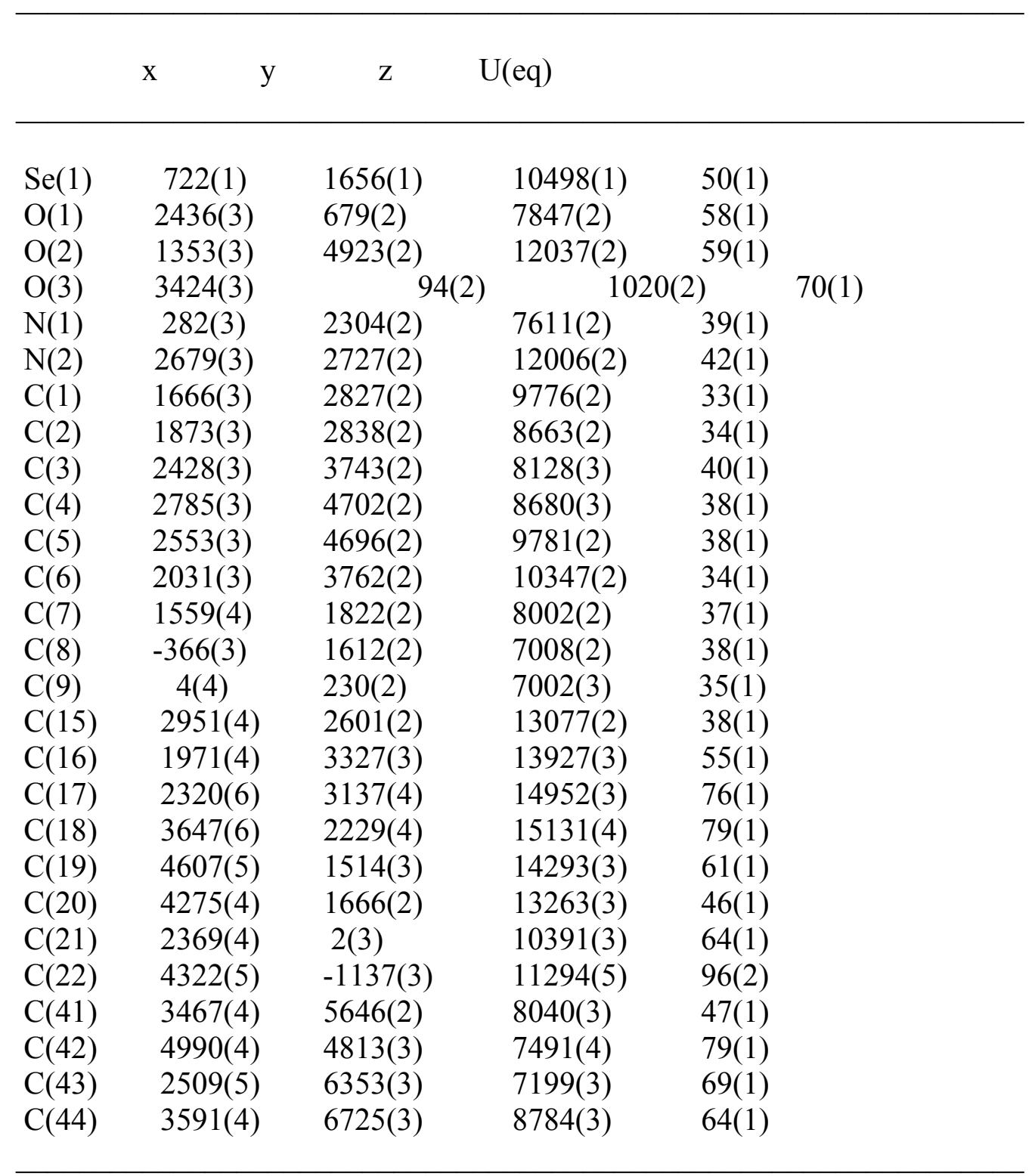


Table 3. Bond lengths [A] and angles [deg] for 32 .

\begin{tabular}{|c|c|}
\hline $\mathrm{Se}(1)-\mathrm{C}(21)$ & $1.925(3)$ \\
\hline $\operatorname{Se}(1)-C(1)$ & $1.927(2)$ \\
\hline $\mathrm{O}(1)-\mathrm{C}(7)$ & $1.222(3)$ \\
\hline $\mathrm{O}(2)-\mathrm{C}(14)$ & $1.223(3)$ \\
\hline $\mathrm{O}(3)-\mathrm{C}(22)$ & $1.373(4)$ \\
\hline $\mathrm{O}(3)-\mathrm{C}(21)$ & $1.415(4)$ \\
\hline $\mathrm{N}(1)-\mathrm{C}(7)$ & $1.339(4)$ \\
\hline $\mathrm{N}(1)-\mathrm{C}(8)$ & $1.410(4)$ \\
\hline $\mathrm{N}(2)-\mathrm{C}(14)$ & $1.343(3)$ \\
\hline $\mathrm{N}(2)-\mathrm{C}(15)$ & $1.409(4)$ \\
\hline $\mathrm{C}(1)-\mathrm{C}(6)$ & $1.401(4)$ \\
\hline $\mathrm{C}(1)-\mathrm{C}(2)$ & $1.389(4)$ \\
\hline$C(2)-C(3)$ & $1.375(4)$ \\
\hline$C(2)-C(7)$ & $1.509(4)$ \\
\hline$C(3)-C(4)$ & $1.405(4)$ \\
\hline$C(4)-C(5)$ & $1.373(4)$ \\
\hline$C(4)-C(41)$ & $1.537(4)$ \\
\hline$C(5)-C(6)$ & $1.401(3)$ \\
\hline $\mathrm{C}(6)-\mathrm{C}(14)$ & $1.491(4)$ \\
\hline$C(8)-C(13)$ & $1.374(4)$ \\
\hline $\mathrm{C}(8)-\mathrm{C}(9)$ & $1.384(3)$ \\
\hline C(9)-C(10) & $1.377(4)$ \\
\hline $\mathrm{C}(10)-\mathrm{C}(11)$ & $1.345(5)$ \\
\hline $\mathrm{C}(11)-\mathrm{C}(12)$ & $1.379(4)$ \\
\hline$C(12)-C(13)$ & $1.372(5)$ \\
\hline$C(15)-C(16)$ & $1.374(5)$ \\
\hline$C(15)-C(20)$ & $1.385(4)$ \\
\hline$C(16)-C(17)$ & $1.378(5)$ \\
\hline $\mathrm{C}(17)-\mathrm{C}(18)$ & $1.373(6)$ \\
\hline $\mathrm{C}(18)-\mathrm{C}(19)$ & $1.354(6)$ \\
\hline$C(19)-C(20)$ & $1.376(5)$ \\
\hline $\mathrm{C}(41)-\mathrm{C}(43)$ & $1.529(5)$ \\
\hline$C(41)-C(42)$ & $1.518(5)$ \\
\hline$C(41)-C(44)$ & $1.538(5)$ \\
\hline $\mathrm{C}(21)-\mathrm{SE}(1)-\mathrm{C}(1)$ & $99.98(14)$ \\
\hline $\mathrm{C}(22)-\mathrm{O}(3)-\mathrm{C}(21)$ & $112.9(3)$ \\
\hline $\mathrm{C}(7)-\mathrm{N}(1)-\mathrm{C}(8)$ & $128.3(2)$ \\
\hline$C(14)-N(2)-C(15)$ & $127.1(2)$ \\
\hline $\mathrm{C}(6)-\mathrm{C}(1)-\mathrm{C}(2)$ & $118.6(2)$ \\
\hline $\mathrm{C}(6)-\mathrm{C}(1)-\mathrm{SE}(1)$ & $121.5(2)$ \\
\hline $\mathrm{C}(2)-\mathrm{C}(1)-\mathrm{SE}(1)$ & $119.62(19)$ \\
\hline
\end{tabular}




$\begin{array}{lc}\mathrm{C}(3)-\mathrm{C}(2)-\mathrm{C}(1) & 120.9(2) \\ \mathrm{C}(3)-\mathrm{C}(2)-\mathrm{C}(7) & 117.9(3) \\ \mathrm{C}(1)-\mathrm{C}(2)-\mathrm{C}(7) & 121.2(2) \\ \mathrm{C}(2)-\mathrm{C}(3)-\mathrm{C}(4) & 121.6(3) \\ \mathrm{C}(5)-\mathrm{C}(4)-\mathrm{C}(3) & 116.9(2) \\ \mathrm{C}(5)-\mathrm{C}(4)-\mathrm{C}(41) & 123.7(3) \\ \mathrm{C}(3)-\mathrm{C}(4)-\mathrm{C}(41) & 119.3(3) \\ \mathrm{C}(4)-\mathrm{C}(5)-\mathrm{C}(6) & 122.7(2) \\ \mathrm{C}(5)-\mathrm{C}(6)-\mathrm{C}(1) & 119.1(3) \\ \mathrm{C}(5)-\mathrm{C}(6)-\mathrm{C}(14) & 115.0(2) \\ \mathrm{C}(1)-\mathrm{C}(6)-\mathrm{C}(14) & 125.9(2) \\ \mathrm{O}(1)-\mathrm{C}(7)-\mathrm{N}(1) & 125.3(3) \\ \mathrm{O}(1)-\mathrm{C}(7)-\mathrm{C}(2) & 120.9(3) \\ \mathrm{N}(1)-\mathrm{C}(7)-\mathrm{C}(2) & 113.9(2) \\ \mathrm{C}(13)-\mathrm{C}(8)-\mathrm{C}(9) & 119.1(3) \\ \mathrm{C}(13)-\mathrm{C}(8)-\mathrm{N}(1) & 118.1(2) \\ \mathrm{C}(9)-\mathrm{C}(8)-\mathrm{N}(1) & 122.8(3) \\ \mathrm{C}(8)-\mathrm{C}(9)-\mathrm{C}(10) & 119.4(3) \\ \mathrm{C}(11)-\mathrm{C}(10)-\mathrm{C}(9) & 121.8(3) \\ \mathrm{C}(10)-\mathrm{C}(11)-\mathrm{C}(12) & 118.9(3) \\ \mathrm{C}(11)-\mathrm{C}(12)-\mathrm{C}(13) & 120.6(3) \\ \mathrm{C}(8)-\mathrm{C}(13)-\mathrm{C}(12) & 120.2(3) \\ \mathrm{O}(2)-\mathrm{C}(14)-\mathrm{N}(2) & 121.9(3) \\ \mathrm{O}(2)-\mathrm{C}(14)-\mathrm{C}(6) & 121.5(2) \\ \mathrm{N}(2)-\mathrm{C}(14)-\mathrm{C}(6) & 116.3(2) \\ \mathrm{C}(16)-\mathrm{C}(15)-\mathrm{C}(20) & 119.5(3) \\ \mathrm{C}(16)-\mathrm{C}(15)-\mathrm{N}(2) & 123.7(3) \\ \mathrm{C}(20)-\mathrm{C}(15)-\mathrm{N}(2) & 116.8(3) \\ \mathrm{C}(17)-\mathrm{C}(16)-\mathrm{C}(15) & 120.0(4) \\ \mathrm{C}(18)-\mathrm{C}(17)-\mathrm{C}(16) & 120.3(4) \\ \mathrm{C}(19)-\mathrm{C}(18)-\mathrm{C}(17) & 119.5(4) \\ \mathrm{C}(18)-\mathrm{C}(19)-\mathrm{C}(20) & 121.4(4) \\ \mathrm{C}(19)-\mathrm{C}(20)-\mathrm{C}(15) & 119.2(3) \\ \mathrm{O}(3)-\mathrm{C}(21)-\mathrm{SE}(1) & 110.5(2) \\ \mathrm{C}(43)-\mathrm{C}(41)-\mathrm{C}(42) & 109.8(3) \\ \mathrm{C}(43)-\mathrm{C}(41)-\mathrm{C}(44) & 108.2(2) \\ \mathrm{C}(42)-\mathrm{C}(41)-\mathrm{C}(44) & 109.5(3) \\ \mathrm{C}(43)-\mathrm{C}(41)-\mathrm{C}(4) & 110.2(3) \\ \mathrm{C}(42)-\mathrm{C}(41)-\mathrm{C}(4) & 108.3(2) \\ \mathrm{C}(44)-\mathrm{C}(41)-\mathrm{C}(4) & 110.8(3) \\ & \end{array}$

Symmetry transformations used to generate equivalent atoms: 
Table 4. Anisotropic displacement parameters $\left(\AA^{2} \times 10^{3}\right)$ for 32. The anisotropic displacement factor exponent takes the form: $-2 \mathrm{p}^{2}\left[\mathrm{~h}^{2} \mathrm{a}^{* 2} \mathrm{U}^{11}+\ldots+2 \mathrm{hk} \mathrm{a}^{*} \mathrm{~b}^{*} \mathrm{U}^{12}\right]$

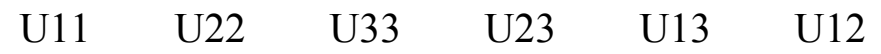

\begin{tabular}{lllllll}
\hline & & & & \\
$\mathrm{Se}(1)$ & $55(1)$ & $48(1)$ & $54(1)$ & $5(1)$ & $-9(1)$ & $-28(1)$ \\
$\mathrm{O}(1)$ & $50(2)$ & $40(1)$ & $79(2)$ & $-19(1)$ & $-28(1)$ & $0(1)$ \\
$\mathrm{O}(2)$ & $83(2)$ & $29(1)$ & $50(1)$ & $-8(1)$ & $-22(1)$ & $5(1)$ \\
$\mathrm{O}(3)$ & $89(2)$ & $42(1)$ & $85(2)$ & $4(1)$ & $-39(2)$ & $-19(1)$ \\
$\mathrm{N}(1)$ & $42(2)$ & $27(1)$ & $45(2)$ & $-5(1)$ & $-16(1)$ & $-6(1)$ \\
$\mathrm{N}(2)$ & $56(2)$ & $27(1)$ & $36(2)$ & $-2(1)$ & $-15(1)$ & $-4(1)$ \\
$\mathrm{C}(1)$ & $30(2)$ & $26(1)$ & $41(2)$ & $0(1)$ & $-10(1)$ & $-7(1)$ \\
$\mathrm{C}(2)$ & $31(2)$ & $30(1)$ & $41(2)$ & $-3(1)$ & $-14(1)$ & $-7(1)$ \\
$\mathrm{C}(3)$ & $46(2)$ & $39(1)$ & $39(2)$ & $2(1)$ & $-13(2)$ & $-16(1)$ \\
$\mathrm{C}(4)$ & $35(2)$ & $36(1)$ & $45(2)$ & $4(1)$ & $-12(2)$ & $-12(1)$ \\
$\mathrm{C}(5)$ & $43(2)$ & $26(1)$ & $48(2)$ & $0(1)$ & $-16(2)$ & $-14(1)$ \\
$\mathrm{C}(6)$ & $34(2)$ & $27(1)$ & $38(2)$ & $-2(1)$ & $-12(1)$ & $-3(1)$ \\
$\mathrm{C}(7)$ & $38(2)$ & $35(1)$ & $38(2)$ & $-1(1)$ & $-11(2)$ & $-11(1)$ \\
$\mathrm{C}(8)$ & $39(2)$ & $41(1)$ & $36(2)$ & $-4(1)$ & $-9(2)$ & $-16(1)$ \\
$\mathrm{C}(9)$ & $60(2)$ & $36(1)$ & $60(2)$ & $3(1)$ & $-21(2)$ & $-22(1)$ \\
$\mathrm{C}(10)$ & $70(3)$ & $54(2)$ & $77(3)$ & $-4(2)$ & $-22(2)$ & $-35(2)$ \\
$\mathrm{C}(11)$ & $72(3)$ & $81(2)$ & $73(3)$ & $-12(2)$ & $-23(2)$ & $-46(2)$ \\
$\mathrm{C}(12)$ & $53(3)$ & $81(2)$ & $70(3)$ & $1(2)$ & $-32(2)$ & $-21(2)$ \\
$\mathrm{C}(13)$ & $49(2)$ & $48(2)$ & $64(2)$ & $-2(2)$ & $-23(2)$ & $-12(2)$ \\
$\mathrm{C}(14)$ & $37(2)$ & $27(1)$ & $42(2)$ & $-4(1)$ & $-12(1)$ & $-8(1)$ \\
$\mathrm{C}(15)$ & $50(2)$ & $34(1)$ & $35(2)$ & $2(1)$ & $-10(2)$ & $-22(1)$ \\
$\mathrm{C}(16)$ & $64(3)$ & $51(2)$ & $43(2)$ & $-2(2)$ & $-8(2)$ & $-12(2)$ \\
$\mathrm{C}(17)$ & $105(4)$ & $78(2)$ & $38(2)$ & $-6(2)$ & $-9(2)$ & $-23(2)$ \\
$\mathrm{C}(18)$ & $117(4)$ & $82(3)$ & $48(3)$ & $8(2)$ & $-37(3)$ & $-35(3)$ \\
$\mathrm{C}(19)$ & $71(3)$ & $60(2)$ & $64(3)$ & $18(2)$ & $-33(2)$ & $-27(2)$ \\
$\mathrm{C}(20)$ & $54(2)$ & $37(1)$ & $50(2)$ & $11(1)$ & $-16(2)$ & $-19(1)$ \\
$\mathrm{C}(21)$ & $87(3)$ & $36(2)$ & $73(3)$ & $4(2)$ & $-28(2)$ & $-20(2)$ \\
$\mathrm{C}(22)$ & $83(4)$ & $54(2)$ & $137(5)$ & $-1(2)$ & $-45(3)$ & $7(2)$ \\
$\mathrm{C}(41)$ & $50(2)$ & $39(1)$ & $56(2)$ & $3(1)$ & $-4(2)$ & $-21(1)$ \\
$\mathrm{C}(42)$ & $57(3)$ & $63(2)$ & $111(4)$ & $-5(2)$ & $19(3)$ & $-25(2)$ \\
$\mathrm{C}(43)$ & $96(3)$ & $57(2)$ & $58(2)$ & $17(2)$ & $-10(2)$ & $-30(2)$ \\
$\mathrm{C}(44)$ & $78(3)$ & $56(2)$ & $71(3)$ & $-3(2)$ & $-2(2)$ & $-42(2)$ \\
& & & & & & \\
\hline & & & & & & \\
\end{tabular}


Table 5. Hydrogen coordinates ( $\left.\times 10^{4}\right)$ and isotropic displacement parameters $\left(\AA^{2} \times 10^{3}\right)$ for 32 .

$\begin{array}{ccccc}\text { Atom } & \mathrm{x} & \mathrm{y} & \mathrm{z} & \mathrm{U}(\text { iso })\left[\AA^{2}\right] \\ ---- & --- & -- & -- & ----- \\ \text { H1 } & 1.02047 & 0.68575 & 0.22561 & 0.0457 \\ \mathrm{H} 2 & 0.69805 & 0.79959 & -0.16101 & 0.0477 \\ \mathrm{H} 3 & 0.74305 & 0.62802 & 0.26175 & 0.0491 \\ \mathrm{H} 5 & 0.72506 & 0.46599 & -0.01656 & 0.0470 \\ \mathrm{H} 9 & 0.92627 & 1.02896 & 0.26194 & 0.0624 \\ \mathrm{H} 10 & 1.04770 & 1.12970 & 0.35673 & 0.0805 \\ \text { H11 } & 1.22589 & 1.00699 & 0.45159 & 0.0904 \\ \text { H12 } & 1.28813 & 0.77605 & 0.45146 & 0.0817 \\ \text { H13 } & 1.17066 & 0.67160 & 0.35656 & 0.0644 \\ \text { H16 } & 0.89267 & 0.60530 & -0.38089 & 0.0634 \\ \text { H17 } & 0.83476 & 0.63749 & -0.55263 & 0.0887 \\ \text { H18 } & 0.61151 & 0.78938 & -0.58229 & 0.0987 \\ \text { H19 } & 0.44895 & 0.90924 & -0.44149 & 0.0777 \\ \text { H20 } & 0.50669 & 0.88540 & -0.26982 & 0.0569 \\ \text { H21A } & 0.79717 & 1.07285 & -0.06338 & 0.0783 \\ \text { H21B } & 0.71795 & 1.01904 & 0.03525 & 0.0783 \\ \text { H22A } & 0.62577 & 1.15769 & -0.17316 & 0.1373 \\ \text { H22B } & 0.49540 & 1.10202 & -0.16865 & 0.1373 \\ \text { H22C } & 0.51934 & 1.16762 & -0.06553 & 0.1373 \\ \text { H42A } & 0.50881 & 0.58607 & 0.29728 & 0.1159 \\ \text { H42B } & 0.44066 & 0.56122 & 0.19771 & 0.1159 \\ \text { H42C } & 0.45731 & 0.46159 & 0.29233 & 0.1159 \\ \text { H43A } & 0.71323 & 0.29713 & 0.31266 & 0.1054 \\ \text { H43B } & 0.84900 & 0.32341 & 0.24626 & 0.1054 \\ \text { H43C } & 0.74517 & 0.42907 & 0.33404 & 0.1054 \\ \text { H44A } & 0.60331 & 0.26678 & 0.16357 & 0.1026 \\ \text { H44B } & 0.57531 & 0.36975 & 0.07142 & 0.1026 \\ \text { H44C } & 0.73641 & 0.27876 & 0.08328 & 0.1026\end{array}$


Figure S25. PLUTON view of Compound 26 showing intermolecular C-H...Se interaction
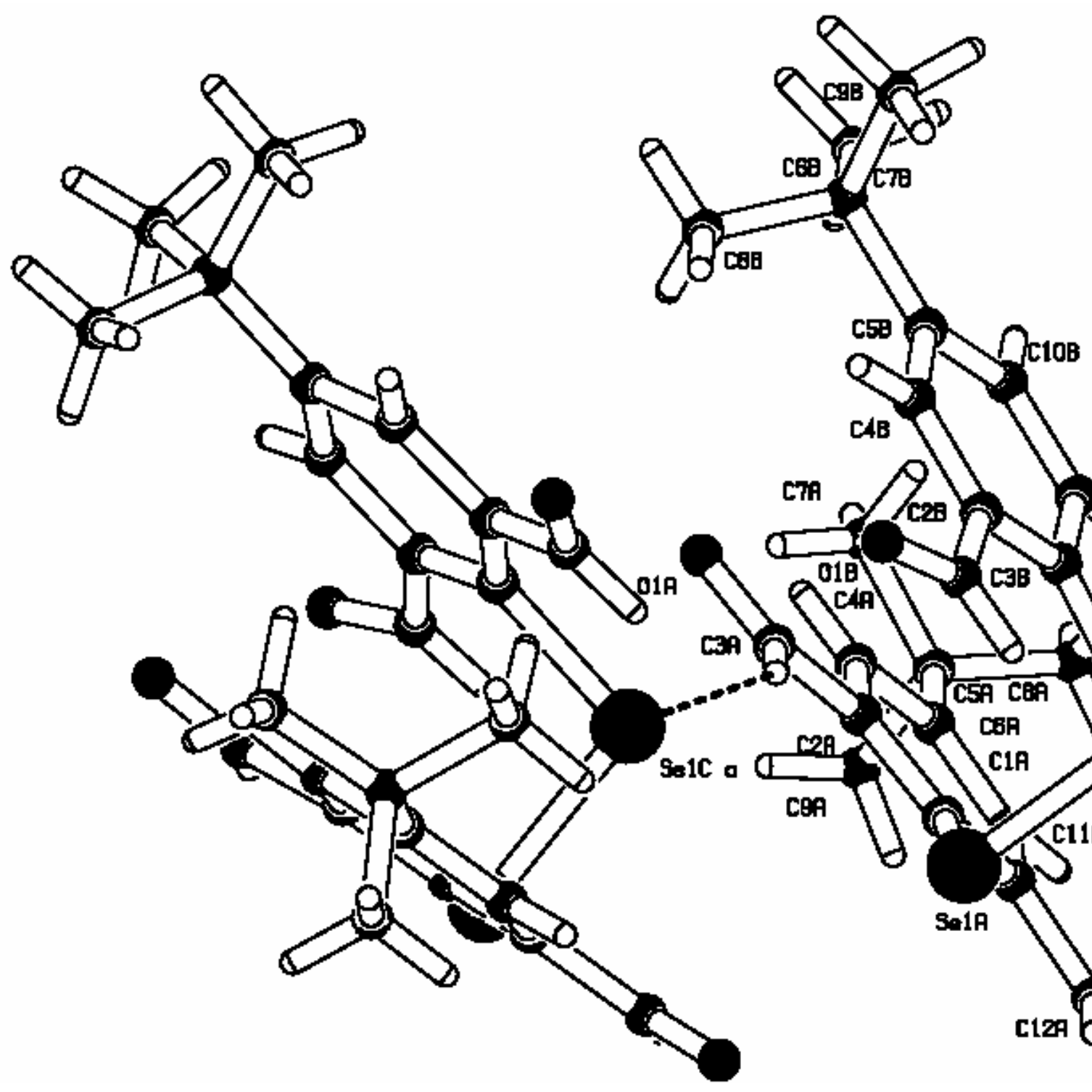
Figure S26. PLUTON view of Compound 34

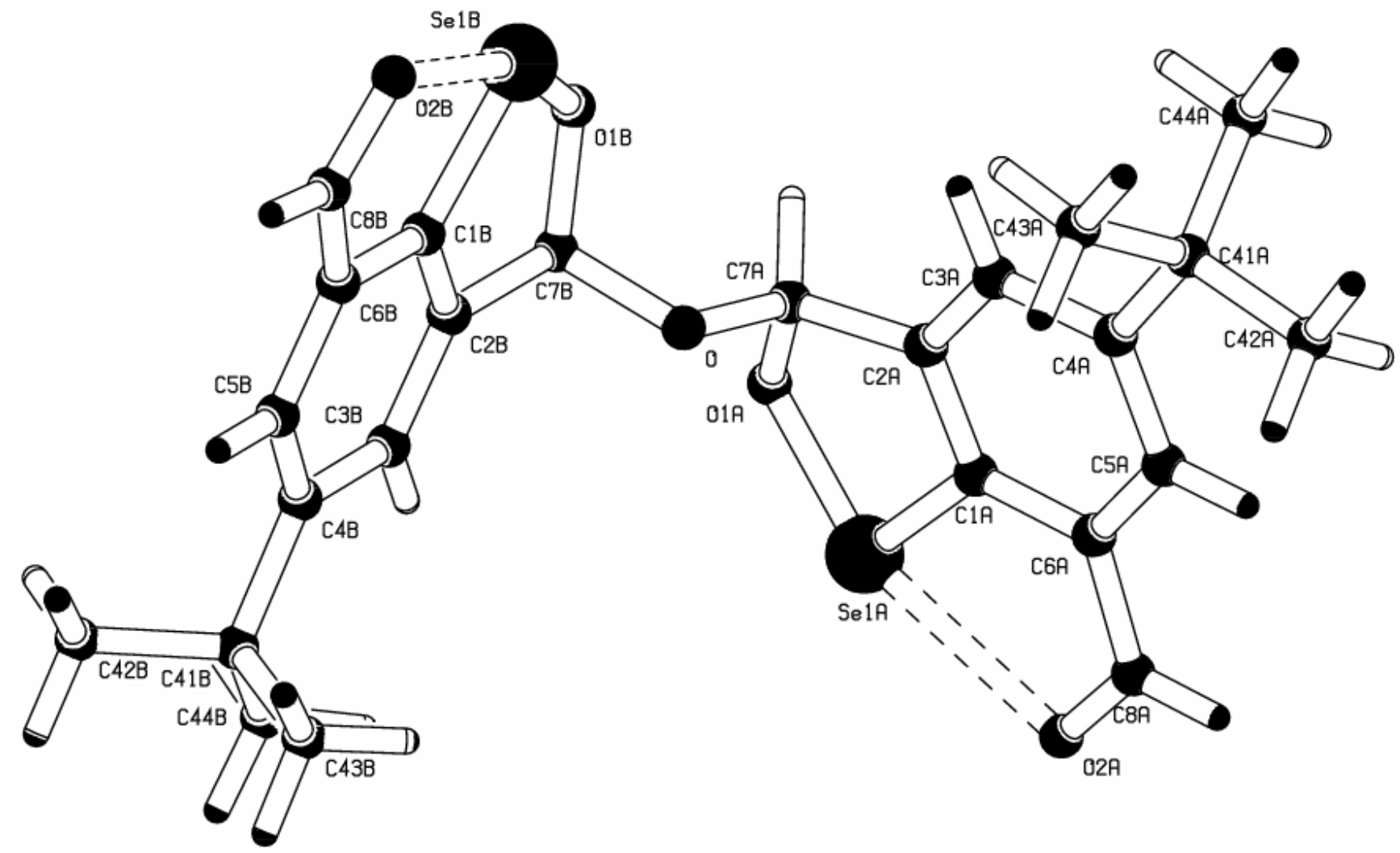


Figure S27: Packing diagram of compound 27

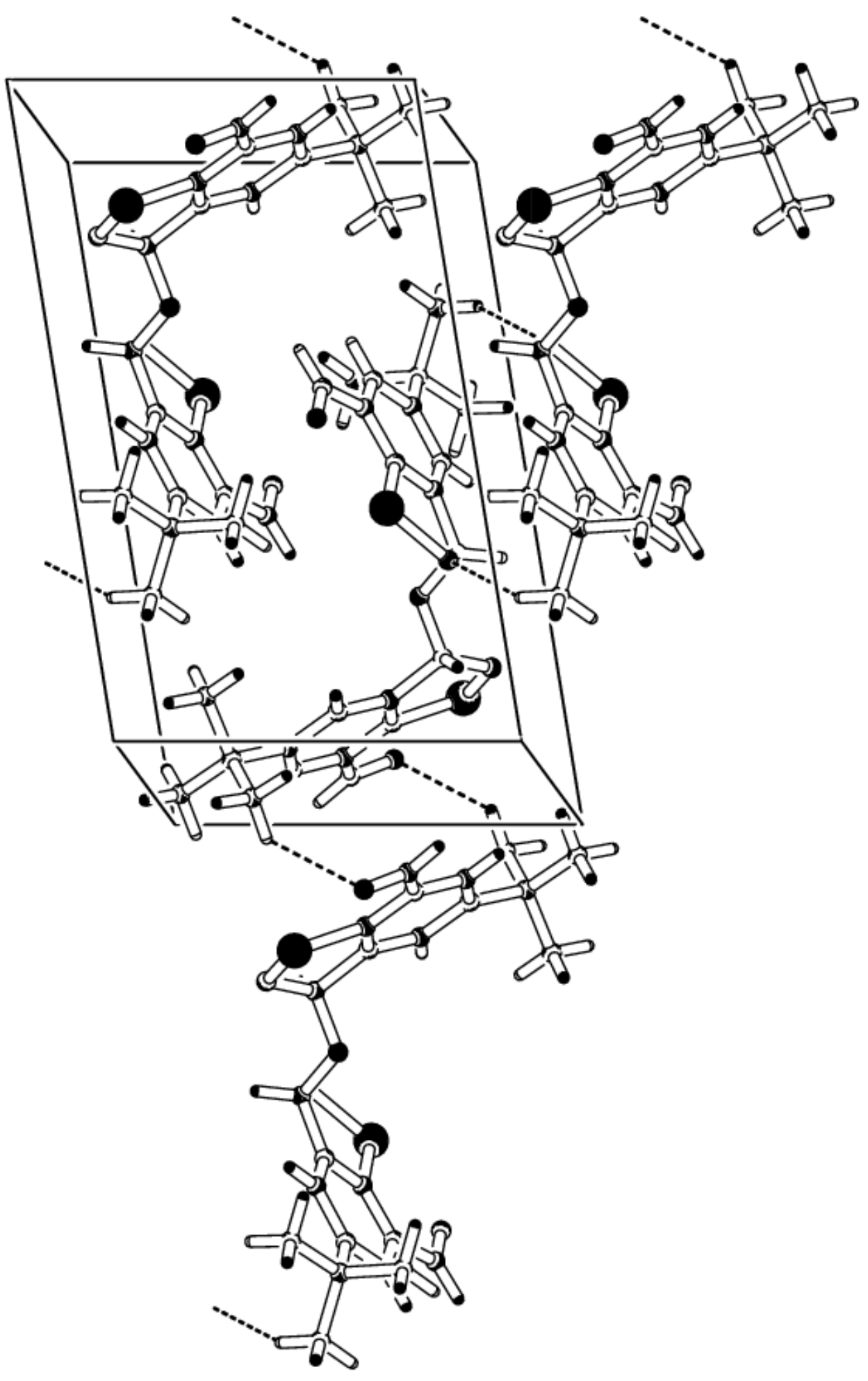


Figure S28. ORTEP diagram of Compound 27

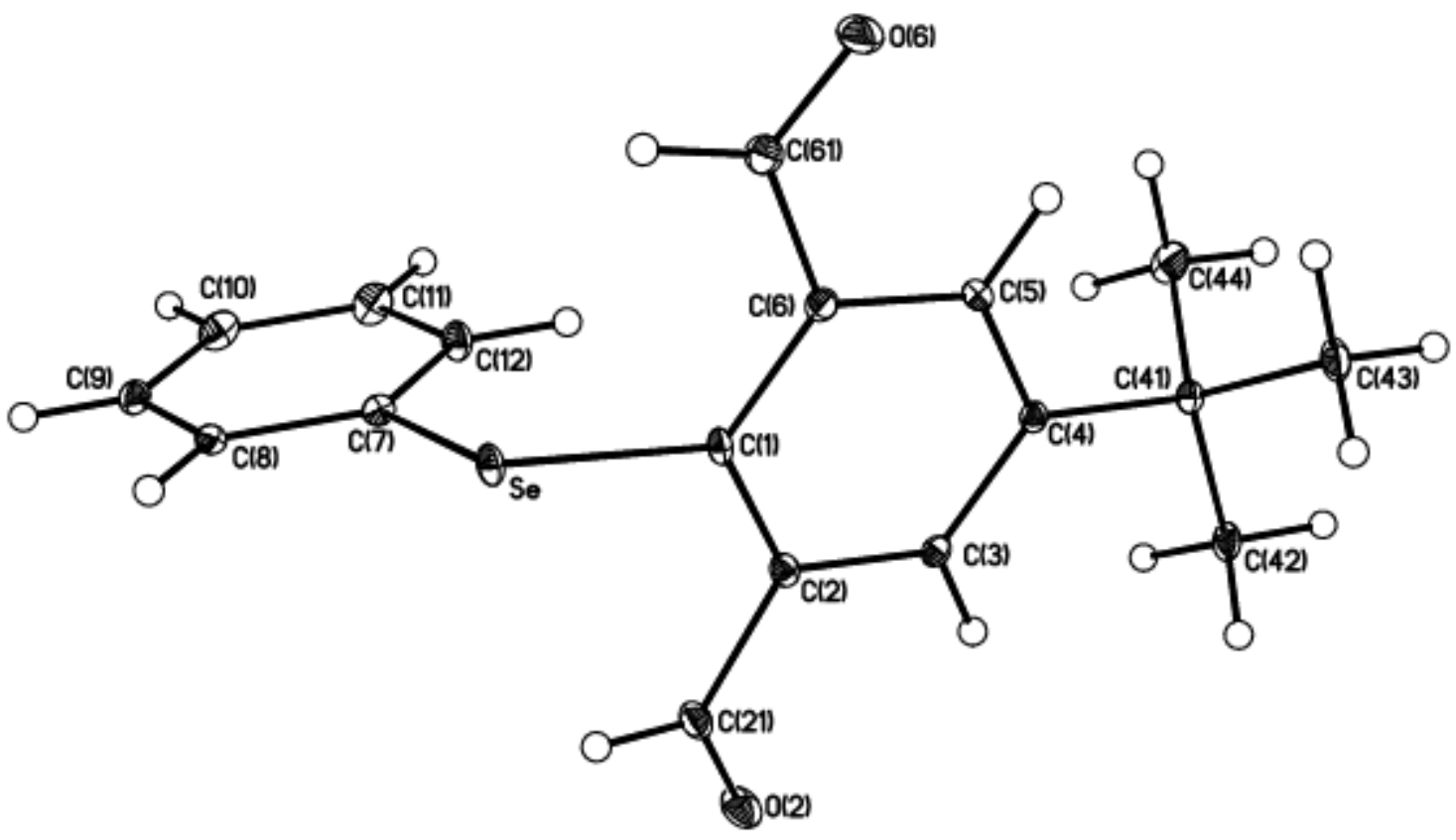


Plot of Se...O distances verses 77Se NMR chemical shifts

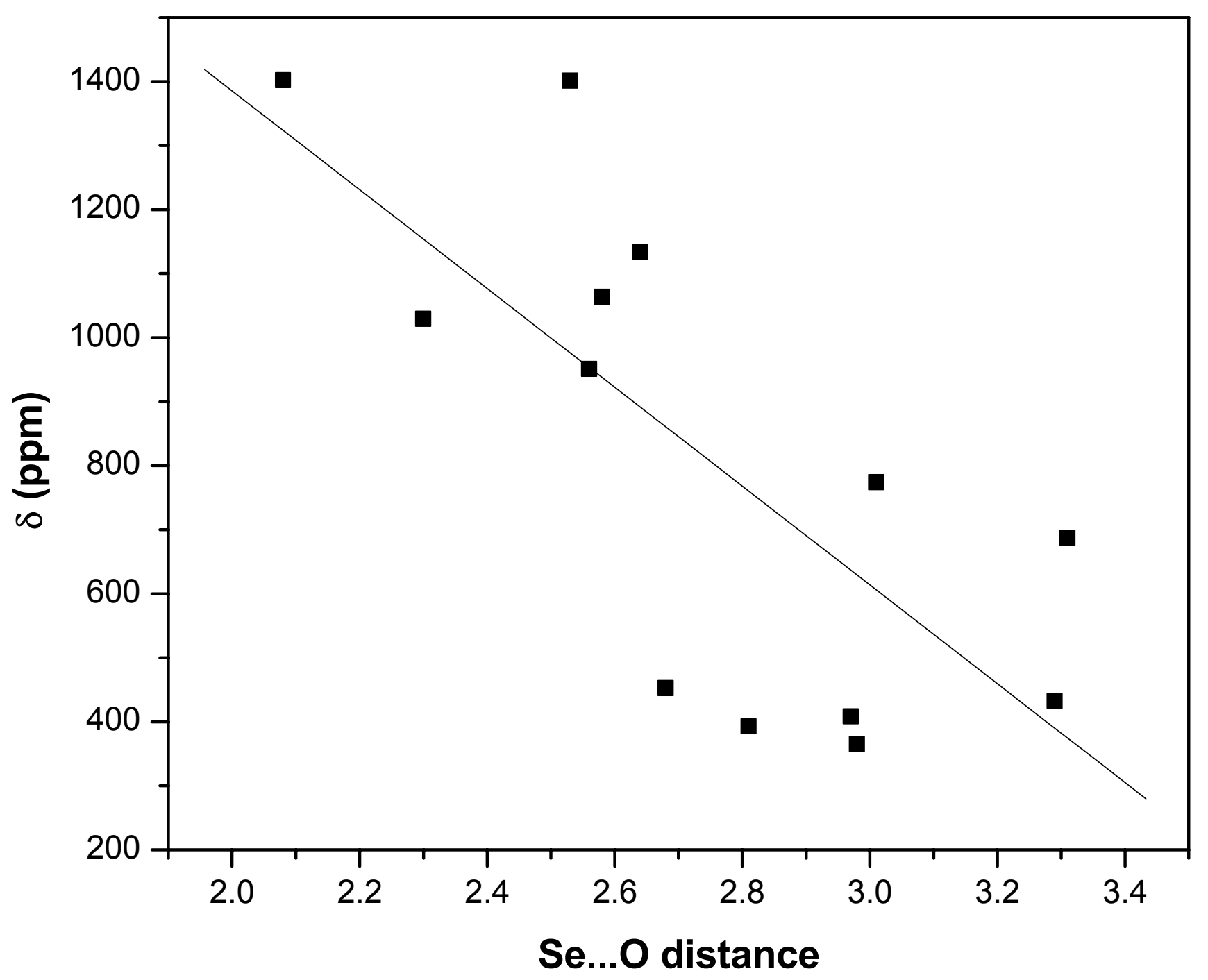

